Monodromy of certain Painlevé-VI transcendents and reflection groups

Dubrovin, Boris and Mazzocco, Marta

2000

MIMS EPrint: 2006.6

Manchester Institute for Mathematical Sciences

School of Mathematics

The University of Manchester

\footnotetext{
Reports available from: http://eprints.maths.manchester.ac.uk/

And by contacting: The MIMS Secretary

School of Mathematics

The University of Manchester

Manchester, M13 9PL, UK
} 


\title{
MONODROMY OF CERTAIN PAINLEVE'-VI TRANSCENDENTS AND REFLECTION GROUPS
}

\author{
B. Dubrovin and M. Mazzocco \\ International School of Advanced Studies, SISSA-ISAS, Trieste.
}

\begin{abstract}
We study the global analytic properties of the solutions of a particular family of Painlevé VI equations with the parameters $\beta=\gamma=0, \delta=\frac{1}{2}$ and $\alpha$ arbitrary. We introduce a class of solutions having critical behaviour of algebraic type, and completely compute the structure of the analytic continuation of these solutions in terms of an auxiliary reflection group in the three dimensional space. The analytic continuation is given in terms of an action of the braid group on the triples of generators of the reflection group. This result is used to classify all the algebraic solutions of our Painlevé VI equation.
\end{abstract}

SISSA preprint no. 149/97/FM, 28 November 1997, revised 8 June 1998. 


\section{INTRODUCTION}

In this paper, we will study the structure of the analytic continuation of the solutions of the following differential equation

$$
\begin{aligned}
y_{x x}= & \frac{1}{2}\left(\frac{1}{y}+\frac{1}{y-1}+\frac{1}{y-x}\right) y_{x}^{2}-\left(\frac{1}{x}+\frac{1}{x-1}+\frac{1}{y-x}\right) y_{x} \\
& +\frac{1}{2} \frac{y(y-1)(y-x)}{x^{2}(x-1)^{2}}\left[(2 \mu-1)^{2}+\frac{x(x-1)}{(y-x)^{2}}\right]
\end{aligned}
$$

in the complex plane, $\mu$ is an arbitrary complex parameter satisfying the condition $2 \mu \notin \mathbb{Z}$.

This is a particular case of the general Painlevé VI equation (see [Ince]) $\operatorname{PVI}(\alpha, \beta, \gamma, \delta)$, that depends on four parameters $\alpha, \beta, \gamma, \delta$, specified by the following choice of the parameters:

$$
\alpha=\frac{(2 \mu-1)^{2}}{2}, \quad \beta=\gamma=0 \quad \delta=\frac{1}{2} .
$$

The general solution $y\left(x ; c_{1}, c_{2}\right)$ of $\operatorname{PVI}(\alpha, \beta, \gamma, \delta)$ satisfies the following two important properties (see [Pain]):

1) The solution $y\left(x ; c_{1}, c_{2}\right)$ can be analytically continued to a meromorphic function on the universal covering of $\overline{\mathbb{C}} \backslash\{0,1, \infty\}$.

2) For generic values of the integration constants $c_{1}, c_{2}$ and of the parameters $\alpha, \beta, \gamma, \delta$, the solution $y\left(x ; c_{1}, c_{2}\right)$ can not be expressed via elementary or classical transcendental functions.

The former claim is the so-called Painlevé property of the equation $\operatorname{PVI}(\alpha, \beta, \gamma, \delta)$, i.e. its solutions $y\left(x ; c_{1}, c_{2}\right)$ may have complicated singularities only at the critical points of the equation, $0,1, \infty$, the position of which does not depend on the choice of the particular solution (the so-called fixed singularities), and all the other singularities of the solution are poles. Positions of the poles depend on the integration constants (the so-called movable singularities).

All the second order ordinary differential equations of the type:

$$
y_{x x}=\mathcal{R}\left(x, y, y_{x}\right),
$$

where $\mathcal{R}$ is rational in $y_{x}$ and meromorphic in $x$ and $y$ and satisfies the Painlevé property of absence of movable critical singularities, were classified by Painlevé and Gambier (see [Pain], and [Gamb]). Only six of these equations, which are given in the Painlevé-Gambier list, satisfy the property 2), i.e. they can not be reduced to known differential equations for elementary and classical special functions. The solutions of these equations define some new functions, the so-called Painlevé transcendents. $\operatorname{PVI}(\alpha, \beta, \gamma, \delta)$ is the most general equation of Painlevé-Gambier list. Indeed all the others can be obtained from $\operatorname{PVI}(\alpha, \beta, \gamma, \delta)$ by a confluence procedure (see [Ince] §14.4).

There are many physical applications of particular solutions of the Painlevé equations which we do not discuss here. We mention only the paper [Tod] where our PVI $\mu$ appears in the problem of the construction of self-dual Bianchi-type IX Einstein metrics, and the 
paper [Dub] where the same equation was used to classify the solutions of WDVV equation in $2 D$-topological field theories.

The name of transcendents could be misleading; indeed, for some particular values of $\left(c_{1}, c_{2}, \alpha, \beta, \gamma, \delta\right)$, the solution $y\left(x ; c_{1}, c_{2}\right)$ can be expressed via classical functions. For example Picard (see [Pic] and [Ok]) showed that the general solution of $\operatorname{PVI}\left(0,0,0, \frac{1}{2}\right)$ can be expressed via elliptic functions, and, more recently, Hitchin [Hit] obtained the general solution of $\operatorname{PVI}\left(\frac{1}{8}, \frac{1}{8}, \frac{1}{8}, \frac{3}{8}\right)$ in terms of the Jacobi theta-functions (see also [Man]). Particular examples of classical solutions, that can be expressed via hypergeometric functions, of PVI were first constructed by Lukashevich [Luka]. A general approach to study the classical solutions of PVI was proposed by Okamoto (see [Ok1][Ok2]). One of the main tools of this approach is the symmetry group of PVI: the particular solutions are those being invariant with respect to some symmetry of PVI. The symmetries act in a non trivial way on the space of the parameters $(\alpha, \beta, \gamma, \delta)$. Okamoto described the fundamental region of the action of this symmetry group and showed that all the classical solutions known at that moment, fit into the boundary of this fundamental region.

The theory of the classical solutions of the Painlevé equations was developed by Umemura and Watanabe ([Um], [Um1], [Um2], [Um3], [Wat]); in particular, all the oneparameter families of classical solutions of PVI were classified in [Wat]. Watanabe also proved that, loosely speaking, all the other classical solutions of PVI (i.e. not belonging to the one-parameter families) can only be given by algebraic functions.

Examples of algebraic solutions were found in [Hit1], for PVI $\left(\frac{1}{8},-\frac{1}{8}, \frac{1}{2 k^{2}}, \frac{1}{2}-\frac{1}{2 k^{2}}\right)$, for an arbitrary integer $k$. Other examples for PVI $\mu$ were constructed in [Dub]. They turn out to be related to the group of symmetries of the regular polyhedra in the three dimensional space. Other algebraic solutions of PVI can be extracted from the recent paper [Seg].

The main aim of our work is to elaborate a tool to classify all the algebraic solutions of the Painlevé VI equation (for the other five Painlevé equations, algebraic solutions have been classified, see [Kit], [Wat1], [Mur] and [Mur1]). Our idea is very close to the main idea of the classical paper of Schwartz (see [Schw]) devoted to the classification of the algebraic solutions of the Gauss hypergeometric equation. Let $y\left(x ; c_{1}, c_{2}\right)$ be a branch of a solution of PVI; its analytic continuation along any closed path $\gamma$ avoiding the singularities is a new branch $y\left(x ; c_{1}^{\gamma}, c_{2}^{\gamma}\right)$ with new integration constants $c_{1}^{\gamma}, c_{2}^{\gamma}$. Since all the singularities of the solution on $\overline{\mathbb{C}} \backslash\{0,1, \infty\}$ are poles, the result of the analytic continuation depends only on the homotopy class of the loop $\gamma$ on the Riemann sphere with three punctures. As a consequence, the structure of the analytic continuation is described by an action of the fundamental group:

$$
\gamma \in \overline{\mathbb{C}} \backslash\{0,1, \infty\}, \quad \gamma:\left(c_{1}, c_{2}\right) \rightarrow\left(c_{1}^{\gamma}, c_{2}^{\gamma}\right) .
$$

To classify all the algebraic solutions of Painlevé VI, all the finite orbits of this action must be classified.

Our problem differs from Schwartz's linear analogue, because (0.1) is not a linear representation but a non-linear action of the fundamental group. It is also more involved than the problem of the classification of the algebraic solutions of the other five Painlevé equations, because the PVI is the only equation on the Painlevé-Gambier list having a non-abelian fundamental group of the complement of the critical locus.

Although the main idea seems to work for the general $\operatorname{PVI}(\alpha, \beta, \gamma, \delta)$, we managed 
to completely describe the action (0.1), and to solve the problem of the classification of the algebraic solutions, only for the particular one-parameter family PVI $\mu$. Nevertheless, we have decided to publish these results separately, postponing the investigation of the general case to another paper (in an effort to keep the paper within a reasonable size, we also postpone the study of the resonant case $2 \mu \in \mathbb{Z}$, see [Ma]). One of the motivations for the present publication is a nice geometrical interpretation of the structure of the analytic continuation (0.1), that seems to disappear in the general PVI equation.

We now outline the main results and describe the structure of the paper. Let us introduce a class of solutions of PVI $\mu$ a-priori containing all the algebraic solutions. We say that a branch of a solution $y\left(x ; c_{1}, c_{2}\right)$ has critical behaviour of algebraic type, if there exist three real numbers $l_{0}, l_{1}, l_{\infty}$ and three non-zero complex numbers $a_{0}, a_{1}, a_{\infty}$, such that

$$
y(x)=\left\{\begin{array}{r}
a_{0} x^{l_{0}}\left(1+\mathcal{O}\left(x^{\varepsilon}\right)\right), \quad \text { as } \quad x \rightarrow 0, \\
1-a_{1}(1-x)^{l_{1}}\left(1+\mathcal{O}\left((1-x)^{\varepsilon}\right)\right), \quad \text { as } \quad x \rightarrow 1, \\
a_{\infty} x^{1-l_{\infty}\left(1+\mathcal{O}\left(x^{-\varepsilon}\right)\right),} \quad \text { as } \quad x \rightarrow \infty,
\end{array}\right.
$$

where $\varepsilon>0$ is small enough. We show that there exists a three-parameter family of solutions of PVI $\mu$ with critical behaviour of algebraic type, where $\mu$ itself is a function of $l_{0}, l_{1}, l_{\infty}$. Of course, for an algebraic solution, the indices $l_{0}, l_{1}, l_{\infty}$ must be rational.

It turns out that the three-parameter family of solutions (0.2) is closed under the analytic continuation (0.1), if and only if $\mu$ is real. One of our main results is the parameterization of the solutions $(0.2)$ by ordered triples of planes in the three dimensional Euclidean space(see Section 1.4). In particular, the indices $l_{0}, l_{1}, l_{\infty}$ are related to the angles $\pi r_{0}, \pi r_{1}, \pi r_{\infty}$ between the planes:

$$
l_{i}=\left\{\begin{array}{r}
2 r_{i} \quad \text { if } \quad 0<r_{i} \leq \frac{1}{2} \\
2-2 r_{i} \quad \text { if } \quad \frac{1}{2} \leq r_{i}<1
\end{array} \quad i=0,1, \infty\right.
$$

and the parameter $\mu$ is determined within the ambiguity $\mu \mapsto \pm \mu+n, n \in \mathbb{Z}$, by the equation:

$$
\sin ^{2} \pi \mu=\cos ^{2} \pi r_{0}+\cos ^{2} \pi r_{1}+\cos ^{2} \pi r_{\infty}+2 \cos \pi r_{0} \cos \pi r_{1} \cos \pi r_{\infty}
$$

This ambiguity and the one due to the reordering of the planes can be absorbed by the symmetries of PVI $\mu$ described in Section 1.2.

We compute the analytic continuation (0.1) in terms of some elementary operations on the planes. This computation leads to prove that, for an algebraic solution of PVI $\mu$, the reflections in the planes must generate the symmetry group of a regular polyhedron in $\mathbb{R}^{3}$. Another result of this paper is the classification of all algebraic solutions of PVI $\mu$. They are in one-to-one correspondence, modulo the symmetries of the equations described in Section 1.2, with the reciprocal pairs of the three-dimensional regular polyhedra and star-polyhedra (the description of the star-polyhedra can be found in [Cox]). The solutions corresponding to the regular tetrahedron, cube and icosahedron are the ones obtained in 
[Dub] using the theory of polynomial Frobenius manifolds. The solutions corresponding to the regular great icosahedron, and regular great dodecahedron are new. Our method not only allows to classify the solutions, but also to obtain the explicit formulae, as we do in Section 2.4 .

The main tool to obtain these results is the isomonodromy deformation method (see [Fuchs], [Sch] and [JMU], [ItN], [FlN]). The Painlevé VI is represented as the equation of isomonodromy deformation of the auxiliary Fuchsian system

$$
\frac{\mathrm{d} Y}{\mathrm{~d} z}=\left(\frac{A_{0}}{z}+\frac{A_{1}}{z-1}+\frac{A_{x}}{z-x}\right) Y .
$$

For PVI $\mu$, the $2 \times 2$ matrices $A_{0}, A_{1}, A_{x}$ are nilpotent and

$$
A_{0}+A_{1}+A_{x}=\left(\begin{array}{cc}
-\mu & 0 \\
0 & \mu
\end{array}\right) \text {. }
$$

The entries of the matrices $A_{i}$ are complicated expressions of $x, y, y_{x}$ and of some quadrature $\int R(x, y) \mathrm{d} x$. The monodromy of (0.3) remains constant if and only if $y=y(x)$ satisfies PVI. Thus, the solutions of PVI $\mu$ are parameterized by the monodromy data of the Fuchsian system (0.3) (see Section 1.1). In section 1.2, we compute the structure of the analytic continuation in terms of the monodromy data. On this basis, in Section 1.3, we classify all the monodromy data of the algebraic solutions of PVI $\mu$. To this end, we classify all the rational solutions of certain trigonometric equations using the method of a paper by Gordan (see [Gor]). In Section 1.4, we parameterize the monodromy data of PVI $\mu$ by ordered triples of planes in the three-dimensional space, considered modulo rotations. The structure of the analytic continuation of the solutions of PVI $\mu$ is reformulated in terms of a certain action of the braid group $B_{3}$ on the triples of planes. The group $G$ generated by the reflections with respect to the planes remains unchanged. For the algebraic solutions, the group $G$ turns out to coincide with the symmetry group of one of the regular polyhedra in the three-dimensional Euclidean space. We also give another proof, suggested by E. Vinberg, of this result, and we establish that the class of solutions of PVI $\mu$ parameterized by triples of planes in the three dimensional Euclidean space is invariant with respect to the analytic continuation.

In the second part of the paper, we identify this class of solutions of PVI $\mu$ with the class of solutions having critical behaviour of algebraic type (0.2). In Section 2.1, we prove that the solution $y(x)$ of the form (0.2), for a fixed value of $\mu$, is uniquely determined by its asymptotic behaviour near one of the critical points, i.e. by any of the pairs $\left(a_{0}, l_{0}\right)$, $\left(a_{1}, l_{1}\right),\left(a_{\infty}, l_{\infty}\right)$. In particular, we prove that, for an algebraic solution of PVI $\mu$, the indices $l_{0}, l_{1}, l_{\infty}$ must satisfy:

$$
0<l_{i} \leq 1, \quad i=0,1, \infty .
$$

To derive the connection formulae establishing the relations between these pairs, we use (see Section 2.3) the properly adapted method of Jimbo (see [Jim]). This method allows to express the monodromy data of the auxiliary Fuchsian system (0.3) in terms of the parameters $\left(a_{0}, l_{0}\right),\left(a_{1}, l_{1}\right)$ or $\left(a_{\infty}, l_{\infty}\right)$. For convenience of the reader, and because of some differences between the assumptions of Jimbo's work and ours, we give a complete 
derivation of the connection formulae in Section 2.2. Using the results of the Sections 1.3 and 1.4, we complete the computation of the critical behaviour (0.2) for all the branches of the analytic continuation of the solution. The result of this computation is used in Section 2.4 to obtain the explicit formulae for the algebraic solutions of PVI $\mu$.

Remark 0.1. The resulting classification of the algebraic solutions of PVI $\mu$ is in striking similarity to the Schwartz's classification (see [Schw]) of the algebraic solutions of the hypergeometric equation. According to Schwartz, the algebraic solutions of the hypergeometric equation, considered modulo contiguity transformations, are of fifteen types (the first type consists of an infinite sequence of solutions). The rows $(2-15)$ of Schwartz's list (see, for example, the table in Section 2.7.2 of [Bat]) correspond to the triples of generating reflections of the symmetry groups of regular polyhedra in the three-dimensional Euclidean space (we are grateful to E. Vinberg for bringing this point to our attention). The parameter $\lambda, \mu, \nu$ of the hypergeometric equation shown in the table are just the angles between the mirrors of the reflections, divided by $\pi$.

According to our classification, the algebraic solutions of PVI $\mu$, considered modulo symmetries, are in one-to-one correspondence to the classes of equivalence of the triples of generating reflections in the symmetry groups of regular polyhedra. The equivalence is defined by an action of the braid group $B_{3}$ on the triples and by orthogonal transformations. We find that in the groups $G=W\left(A_{3}\right)$ and $G=W\left(B_{3}\right)$, the symmetry groups of respectively the regular tetrahedron and of the cube or regular octahedron, there is only one equivalence class of triples of generating reflections; these are given respectively by the rows $(2,3)$ and by $(4,5)$ of Schwartz's table. In the group $W\left(H_{3}\right)$ of symmetries of regular icosahedron or regular dodecahedron, there are three equivalence classes of triples of reflections which are given respectively by the rows $(6,8,13),(11,14,15)$ and $(7,9,10,12)$ of the Schwartz's table and correspond to icosahedron, great icosahedron and great dodecahedron (or to their reciprocal pairs, see $[\mathrm{Cox}]$ ). To establish the correspondence, we associate a standard system of generating reflections to a regular polyhedron in the following way: let $H$ be the center of the polyhedron, $O$ the center of a face, $P$ a vertex of this face and $Q$ the center of an edge of the same face through the vertex $P$. Then the reflections with respect to the planes $H O P, H O Q$ and $H P Q$ are the standard system of generators. Our five algebraic solutions correspond to the classes of equivalence of the standard systems of generators obtained by this contruction applied to tetrahedron, cube, icosahedron, great icosahedron, great dodecahedron.

Summarizing, we see that the list of all the algebraic solutions of PVI $\mu$ is obtained by folding of the list of Schwartz modulo the action of the braid group. This relation between the algebraic solutions of $\mathrm{PVI} \mu$ and the algebraic hypergeometric functions seems to be surprising also from the point of view of the results of Watanabe (see [Wat]) who classified all the one-parameter families of classical solutions of $\mathrm{PVI} \mu$ (essentially, all of them are given by hypergeometric functions). Using these results, one can easily check that our algebraic solutions do not belong to any of the one-parameter families of classical solutions of $\mathrm{PVI} \mu$. 


\section{Acknowledgments}

The authors are indebted to E. Vinberg for the elegant proof of theorem 1.8. We thank A. Akhmedov for a simple proof of the Algebraic Lemma, Section 1.4. We thank also R. Conte for drawing our attention to the classical work of Picard (see [Pic]) and V. Sokolov and F. Zanolin for useful discussions.

\section{STRUCTURE OF ANALYTIC CONTINUATION AND ALGEBRAIC SO- LUTIONS OF PVI $\mu$}

\subsection{Painlevé VI equation as isomonodromy deformation equation.}

In this section we show how the PVI $\mu$ equation can be reduced to the isomonodromy deformation equation of an auxiliary Fuchsian system (see [Sch], [JMU]); moreover we describe the parameterization, essentially due to Schlesinger (see. [Sch]), of the solutions of the PVI $\mu$ equation by the monodromy data of such Fuchsian system.

1.1.1. An auxiliary Fuchsian system and its monodromy data. In this subsection, we introduce an auxiliary Fuchsian system, define its monodromy and connection matrices, and extabilish the correspondence between monodromy matrices and coefficients of the Fuchsian system for a given set of poles.

Let us consider the following Fuchsian system with four regular singularities at $u_{1}$, $u_{2}, u_{3}$ and $\infty$ :

$$
\frac{\mathrm{d}}{\mathrm{d} z} Y=\mathcal{A}(z) Y, \quad z \in \overline{\mathbb{C}} \backslash\left\{u_{1}, u_{2}, u_{3}, \infty\right\}
$$

where $\mathcal{A}(z)$ is a matrix-valued function:

$$
\mathcal{A}(z)=\frac{\mathcal{A}_{1}}{z-u_{1}}+\frac{\mathcal{A}_{2}}{z-u_{2}}+\frac{\mathcal{A}_{3}}{z-u_{3}}
$$

$\mathcal{A}_{i}$ being $2 \times 2$ matrices independent on $z$, and $u_{1}, u_{2}, u_{3}$ being pairwise distinct complex numbers. We assume that the matrices $\mathcal{A}_{i}$ satisfy the following conditions:

$$
\mathcal{A}_{i}^{2}=0 \quad \text { and } \quad \mathcal{A}_{\infty}:=-\mathcal{A}_{1}-\mathcal{A}_{2}-\mathcal{A}_{3}=\left(\begin{array}{cc}
\mu & 0 \\
0 & -\mu
\end{array}\right) .
$$

Indeed, we will see in the latter part of this section that this choice corresponds to the particular case PVI $\mu$ of the Painlevé VI equation. In this paper we consider the nonresonant case $2 \mu \notin \mathbb{Z}$.

The solution $Y(z)$ of the system (1.1) is a multi-valued analytic function in the punctured Riemann sphere $\mathbb{C} \backslash\left\{u_{1}, u_{2}, u_{3}\right\}$ and its multivaluedness is described by the so-called monodromy matrices. 
Let us briefly recall the definition of the monodromy matrices of the Fuchsian system (1.1). First, we fix a basis $\gamma_{1}, \gamma_{2}, \gamma_{3}$ of loops in the fundamental group, with base point at $\infty$, of the punctured Riemann sphere

$$
\pi_{1}\left(\overline{\mathbb{C}} \backslash\left\{u_{1}, u_{2}, u_{3}, \infty\right\}, \hat{x}\right),
$$

and a fundamental matrix for the system (1.1). To fix the basis of the loops, we first perform some cuts between the singularities, namely we cut three parallel segments $\pi_{i}$ between the point at infinity and each $u_{i}$; the segments $\pi_{i}$ are ordered according to the order of the points $u_{1}, u_{2}, u_{3}$, as in the figure 1 . Take $\gamma_{i}$ to be a simple closed curve starting and finishing at infinity, going around $u_{i}$ in positive direction $\left(\gamma_{i}\right.$ is oriented counter-clockwise, $u_{i}$ lies inside, while the other singular points lie outside) and not crossing the cuts $\pi_{i}$. Near $\infty$, we take every loop $\gamma_{i}$ close to the cut $\pi_{i}$ as in the figure 1 .

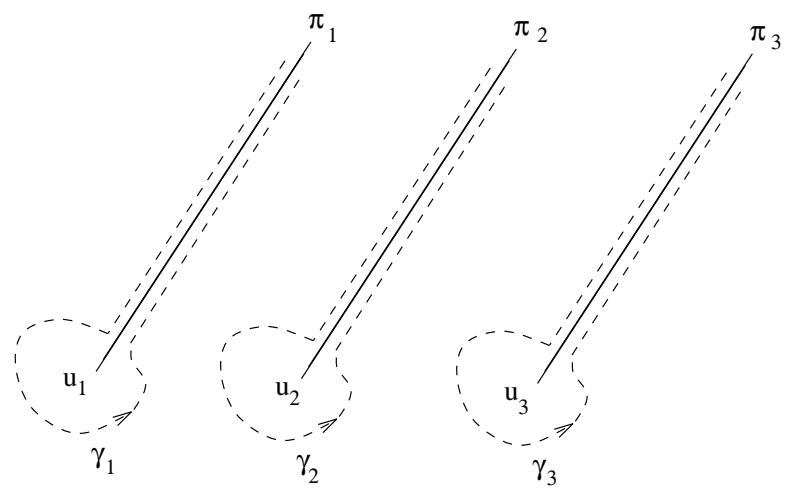

Fig.1. The cuts $\pi_{i}$ between the singularities $u_{i}$ and the oriented loops $\gamma_{i}$.

Now, we fix the fundamental matrix $Y_{\infty}(z)$ of the system (1.1) in such a way that

$$
Y_{\infty}(z)=\left(\mathbf{1}+\mathcal{O}\left(\frac{1}{z}\right)\right)\left(\begin{array}{cc}
z^{-\mu} & 0 \\
0 & z^{\mu}
\end{array}\right), \quad \text { as } \quad z \rightarrow \infty
$$

where $z^{\mu}:=e^{\mu \log z}$, with the choice of the principal branch of the logarithm, with the branch-cut along the common direction of the cuts $\pi_{1}, \pi_{2}, \pi_{3}$. Such a fundamental matrix $Y_{\infty}(z)$ exists and, due to the non-resonance condition, it is uniquely determined. It can be analytically continued to an analytic function on the universal covering of $\overline{\mathbb{C}} \backslash\left\{u_{1}, u_{2}, u_{3}, \infty\right\}$. For any element $\gamma \in \pi_{1}\left(\overline{\mathbb{C}} \backslash\left\{u_{1}, u_{2}, u_{3}, \infty\right\}, \infty\right)$ we denote the result of the analytic continuation of $Y_{\infty}(z)$ along the loop $\gamma$ by $\gamma\left[Y_{\infty}(z)\right]$. Since $\gamma\left[Y_{\infty}(z)\right]$ and $Y_{\infty}(z)$ are two fundamental matrices in the neighborhood of infinity, they must be related by the following relation:

$$
\gamma\left[Y_{\infty}(z)\right]=Y_{\infty}(z) M_{\gamma}
$$

for some constant invertible $2 \times 2$ matrix $M_{\gamma}$ depending only on the homotopy class of $\gamma$. Particularly, the matrix $M_{\infty}:=M_{\gamma_{\infty}}, \gamma_{\infty}$ being a simple loop around infinity in the clock-wise direction, is given by:

$$
M_{\infty}=\left(\begin{array}{cc}
\exp (2 i \pi \mu) & 0 \\
0 & \exp (-2 i \pi \mu)
\end{array}\right) .
$$


The resulting monodromy representation is an anti-homomorphism:

$$
\begin{array}{ccc}
\pi_{1}\left(\overline{\mathbb{C}} \backslash\left\{u_{1}, u_{2}, u_{3}, \infty\right\}, \infty\right) & \rightarrow & S L_{2}(\mathbb{C}) \\
\gamma & \mapsto & M_{\gamma} \\
M_{\gamma \tilde{\gamma}}=M_{\tilde{\gamma}} M_{\gamma} . & &
\end{array}
$$

The images $M_{i}:=M_{\gamma_{i}}$ of the generators $\gamma_{i}, i=1,2,3$ of the fundamental group, are called the monodromy matrices of the Fuchsian system (1.1). They generate the monodromy group of the system, i.e. the image of the representation (1.5). Moreover, due to the fact that, in our particular case, the $\mathcal{A}_{i}$ are nilpotent, satisfy the following relations:

$$
\operatorname{det}\left(M_{i}\right)=1, \quad \operatorname{Tr}\left(M_{i}\right)=2, \quad \text { for } \quad i=1,2,3,
$$

with $M_{i}=\mathbf{1}$ if and only if $\mathcal{A}_{i}=0$. Moreover, since the loop $\left(\gamma_{1} \gamma_{2} \gamma_{3}\right)^{-1}$ is homotopic to $\gamma_{\infty}$, the following relation holds:

$$
M_{\infty} M_{3} M_{2} M_{1}=\mathbf{1}
$$

A simultaneous conjugation $\mathcal{A}_{i} \mapsto D^{-1} \mathcal{A}_{i} D, i=1,2,3$ of the coefficients $\mathcal{A}_{i}$ of the Fuchsian system (1.1) by a diagonal matrix $D$, implies the same conjugation of the monodromy matrices $M_{\gamma} \mapsto D^{-1} M_{\gamma} D$, for any $\gamma \in \pi_{1}\left(\overline{\mathbb{C}} \backslash\left\{u_{1}, u_{2}, u_{3}, \infty\right\}, \infty\right)$.

We now recall the definition of the connection matrices. Let us assume that $M_{i} \neq \mathbf{1}$, or equivalently $\mathcal{A}_{i} \neq 0$, for every $i=1,2,3$. We choose the fundamental matrices $Y_{i}(z)$ of the system (1.1), such that:

$$
Y_{i}=G_{i}\left(\mathbf{1}+\mathcal{O}\left(z-u_{i}\right)\right)\left(z-u_{i}\right)^{J}, \quad \text { as } \quad z \rightarrow u_{i},
$$

where $J$ is the Jordan normal form of $\mathcal{A}_{i}$, namely $J=\left(\begin{array}{ll}0 & 1 \\ 0 & 0\end{array}\right)$, the invertible matrix $G_{i}$ is defined by $\mathcal{A}_{i}=G_{i} J G_{i}^{-1}$, and the choice of the branch of $\log \left(z-u_{i}\right)$ needed in the definition of

$$
\left(z-u_{i}\right)^{J}=\left(\begin{array}{cc}
1 & \log \left(z-u_{i}\right) \\
0 & 1
\end{array}\right)
$$

is similar to the one above. The fundamental matrix $Y_{i}(z)$ is uniquely determined up to the ambiguity:

$$
Y_{i}(z) \mapsto Y_{i}(z) R_{i}
$$

where $R_{i}$ is any matrix commuting with $J$.

Continuing, along, say, the right-hand-side of the cut $\pi_{i}$, the solution $Y_{\infty}$ to a neighborhood of $u_{i}$, we obtain another fundamental matrix around $u_{i}$, that must be related to $Y_{i}(z)$ by:

$$
Y_{\infty}(z)=Y_{i}(z) C_{i}
$$

for some invertible matrix $C_{i}$. The matrices $C_{1}, C_{2}, C_{3}$ are called connection matrices, and are related to the monodromy matrices as follows:

$$
M_{i}=C_{i}^{-1} \exp (2 \pi i J) C_{i}, \quad i=1,2,3 .
$$


Lemma 1.1. Given three matrices $M_{1}, M_{2}, M_{3}, M_{i} \neq \mathbf{1}$ for every $i=1,2,3$, satisfying the relations (1.7) and (1.8), then

i) there exist three matrices $C_{1}, C_{2}, C_{3}$ satisfying the (1.11). Moreover they are uniquely determined by the matrices $M_{1}, M_{2}, M_{3}$, up to the ambiguity $C_{i} \mapsto R_{i}^{-1} C_{i}$, where $R_{i} J=J R_{i}$, for $i=1,2,3$.

ii) If the matrices $M_{1}, M_{2}, M_{3}$ are the monodromy matrices of a Fuchsian system of the form (1.1), then any triple $C_{1}, C_{2}, C_{3}$ satisfying (1.11) can be realized as the connection matrices of the Fuchsian system itself.

Proof. i) By the (1.7), the monodromy matrices have all the eigenvalues equal to one; moreover they can be reduced to the Jordan normal form because $M_{i} \neq \mathbf{1}$. Namely there exists a matrix $\tilde{C}_{i}$ such that:

$$
M_{i}=\tilde{C}_{i}^{-1}\left(\begin{array}{ll}
1 & 1 \\
0 & 1
\end{array}\right) \tilde{C}_{i}
$$

Taking

$$
C_{i}=\left(\begin{array}{cc}
1 & 2 \pi i \\
0 & 1
\end{array}\right) \tilde{C}_{i}
$$

we obtain the needed matrix. Two such matrices $C_{i}$ and $C_{i}^{\prime}$ give the same matrix $M_{i}$ if and only if $C_{i}^{-1} C_{i}^{\prime}$ commutes with $J$, namely if and only if they are related by $C_{i}=R_{i}^{-1} C_{i}^{\prime}$. ii) Let us now assume that $C_{1}^{\prime}, C_{2}^{\prime}, C_{3}^{\prime}$ are the connection matrices of a Fuchsian system of the form (1.1), with monodromy matrices $M_{1}, M_{2}, M_{3}$; id est $Y_{\infty}(z)=Y_{i}^{\prime}(z) C_{i}^{\prime}, i=1,2,3$, for some choice of the solutions $Y_{1}^{\prime}, Y_{2}^{\prime}$ and $Y_{3}^{\prime}$ of the form (1.9). We have

$$
M_{i}=\left(C_{i}^{\prime}\right)^{-1} \exp (2 \pi i J) C_{i}^{\prime}=C_{i}^{-1} \exp (2 \pi i J) C_{i}, \quad i=1,2,3 .
$$

So the matrices $R_{i}=C_{i}^{\prime} C_{i}^{-1}$ must commute with $J$ and $C_{1}, C_{2}, C_{3}$ are the connection matrices with respect to the new solutions $Y_{i}(z)=Y_{i}^{\prime}(z) R_{i}$.

QED

Now, we state the result about the correspondence between monodromy data and coefficients of the Fuchsian system, for a given set of poles:

Lemma 1.2. Two Fuchsian systems (1.1) with the same poles $u_{1}, u_{2}$ and $u_{3}$, and the same value of $\mu$, coincide if and only if they have the same monodromy matrices $M_{1}, M_{2}$, $M_{3}$, with respect to the same basis of the loops $\gamma_{1}, \gamma_{2}$ and $\gamma_{3}$.

Proof. Let $Y_{\infty}^{(1)}(z)$ and $Y_{\infty}^{(2)}(z)$ be the fundamental matrices of the form (1.3) of the two Fuchsian systems. Let us consider the following matrix:

$$
Y(z):=Y_{\infty}^{(2)}(z) Y_{\infty}^{(1)}(z)^{-1} .
$$

$Y(z)$ is an analytic function around infinity:

$$
Y(z)=1+\mathcal{O}\left(\frac{1}{z}\right), \quad \text { as } z \rightarrow \infty
$$


Since the monodromy matrices coincide, $Y(z)$ is a single valued function on $\overline{\mathbb{C}} \backslash\left\{u_{1}, u_{2}, u_{3}\right\}$. Let us prove that $Y(z)$ is analytic also at the points $u_{i}$. Due to Lemma 1.1, we can choose the fundamental matrices $Y_{i}^{(1)}(z)$ and $Y_{i}^{(2)}(z)$ in such a way that

$$
Y_{\infty}^{(1),(2)}(z)=Y_{i}^{(1),(2)}(z) C_{i} \quad i=1,2,3 .
$$

with the same connection matrices $C_{i}$. Then near the point $u_{i}$,

$$
Y(z)=G_{i}^{(2)}\left(\mathbf{1}+\mathcal{O}\left(z-u_{i}\right)\right)\left[G_{i}^{(1)}\left(\mathbf{1}+\mathcal{O}\left(z-u_{i}\right)\right)\right]^{-1} .
$$

This proves that $Y(z)$ is an analytic function on all $\overline{\mathbb{C}}$ and then, by the Liouville theorem

$$
Y(z)=\mathbf{1}
$$

and the two Fuchsian systems must coincide.

Corollary 1.1. Two Fuchsian systems (1.1) with the same poles $u_{1}, u_{2}$ and $u_{3}$, and the same value of $\mu$, are conjugated

$$
\mathcal{A}_{i}^{(1)}=D^{-1} \mathcal{A}_{i}^{(2)} D, \quad i=1,2,3,
$$

with a diagonal matrix $D$, if and only if their monodromy matrices $M_{i}^{(1)}$ and $M_{i}^{(2)}$, with respect to the same basis of the loops $\gamma_{1}, \gamma_{2}$ and $\gamma_{3}$, are conjugated:

$$
M_{i}^{(1)}=D^{-1} M_{i}^{(2)} D, \quad i=1,2,3 .
$$

\subsubsection{The isomonodromy deformations of the Fuchsian system (1.1) and the} Painlevé equation PVI $\mu$. We now want to deform the poles of the Fuchsian system keeping the monodromy fixed. The theory of these deformations is described by the following two results:

Theorem 1.1. Let $M_{1}, M_{2}, M_{3}$ be the monodromy matrices of the Fuchsian system:

$$
\frac{\mathrm{d}}{\mathrm{d} z} Y^{0}=\left(\frac{\mathcal{A}_{1}^{0}}{z-u_{1}^{0}}+\frac{\mathcal{A}_{2}^{0}}{z-u_{2}^{0}}+\frac{\mathcal{A}_{3}^{0}}{z-u_{3}^{0}}\right) Y^{0},
$$

of the above form (1.2), with pairwise distinct poles $u_{i}^{0}$, and with respect to some basis $\gamma_{1}, \gamma_{2}, \gamma_{3}$ of the loops in $\pi_{1}\left(\overline{\mathbb{C}} \backslash\left\{u_{1}^{0}, u_{2}^{0}, u_{3}^{0}, \infty\right\}, \infty\right)$. Then there exists a neighborhood $U \subset \mathbb{C}^{3}$ of the point $u^{0}=\left(u_{1}^{0}, u_{2}^{0}, u_{3}^{0}\right)$ such that, for any $u=\left(u_{1}, u_{2}, u_{3}\right) \in U$, there exists a unique triple $\mathcal{A}_{1}(u), \mathcal{A}_{2}(u), \mathcal{A}_{3}(u)$ of analytic matrix valued functions such that:

$$
\mathcal{A}_{i}\left(u^{0}\right)=\mathcal{A}_{i}^{0}, \quad i=1,2,3,
$$

and the monodromy matrices of the Fuchsian system

$$
\frac{\mathrm{d}}{\mathrm{d} z} Y=A(z ; u) Y=\left(\frac{\mathcal{A}_{1}(u)}{z-u_{1}}+\frac{\mathcal{A}_{2}(u)}{z-u_{2}}+\frac{\mathcal{A}_{3}(u)}{z-u_{3}}\right) Y
$$


with respect to the same basis ${ }^{1} \gamma_{1}, \gamma_{2}, \gamma_{3}$ of the loops, coincide with the given $M_{1}, M_{2}$, $M_{3}$. The matrices $\mathcal{A}_{i}(u)$ are the solutions of the Cauchy problem with the initial data $\mathcal{A}_{i}^{0}$ for the following Schlesinger equations:

$$
\frac{\partial}{\partial u_{j}} \mathcal{A}_{i}=\frac{\left[\mathcal{A}_{i}, \mathcal{A}_{j}\right]}{u_{i}-u_{j}}, \quad \frac{\partial}{\partial u_{i}} \mathcal{A}_{i}=-\sum_{j \neq i} \frac{\left[\mathcal{A}_{i}, \mathcal{A}_{j}\right]}{u_{i}-u_{j}}
$$

The solution $Y_{\infty}^{0}(z)$ of (1.12) of the form (1.3) can be uniquely continued, for $z \neq u_{i}$ $i=1,2,3$, to an analytic function

$$
Y_{\infty}(z, u), \quad u \in U
$$

such that

$$
Y_{\infty}\left(z, u^{0}\right)=Y_{\infty}^{0}(z)
$$

This continuation is the local solution of the Cauchy problem with the initial data $Y_{\infty}^{0}$ for the following system that is compatible to the system (1.13):

$$
\frac{\partial}{\partial u_{i}} Y=-\frac{\mathcal{A}_{i}(u)}{z-u_{i}} Y
$$

Moreover the functions $\mathcal{A}_{i}(u)$ and $Y_{\infty}(z, u)$ can be continued analytically to global meromorphic functions on the universal coverings of

$$
\mathbb{C}^{3} \backslash\{\text { diags }\}:=\left\{\left(u_{1}, u_{2}, u_{3}\right) \in \mathbb{C}^{3} \mid u_{i} \neq u_{j} \text { for } i \neq j\right\}
$$

and

$$
\left\{\left(z, u_{1}, u_{2}, u_{3}\right) \in \mathbb{C}^{4} \mid u_{i} \neq u_{j} \text { for } i \neq j \text { and } z \neq u_{i}, i=1,2,3\right\}
$$

respectively.

The proof can be found, for example, in [Mal], [Miwa], [Sib]. We recall the theorem of solvability of the inverse problem of the monodromy (see [Dek]):

Theorem 1.2. Given three arbitrary matrices, satisfying (1.7) and (1.8), with $M_{\infty}$ of the form (1.4), and given a point $u^{0}=\left(u_{1}^{0}, u_{2}^{0}, u_{3}^{0}\right) \in \mathbb{C}^{3} \backslash\{$ diags $\}$, for any neighborhood $U$ of $u^{0}$, there exist $\left(u_{1}, u_{2}, u_{3}\right) \in U$ and a Fuchsian system of the form (1.1), with the given monodromy matrices, the given $\mu$ and with poles in $u_{1}, u_{2}, u_{3}$.

Remark 1.1. Fuchsian systems of the form (1.1), with coefficients $\mathcal{A}_{i}$ satisfying (1.2), depend on four parameters, one of them being $\mu$. The triples of the monodromy matrices satisfying (1.7) and (1.8), with $M_{\infty}$ of the form (1.4), depend on four parameters too. Loosely speaking, Theorems 1.1 and 1.2 claim that, not only the monodromy matrices are first integrals for the equations of isomonodromy deformation (1.14), but they provide

1 Observe that the basis $\gamma_{1}, \gamma_{2}, \gamma_{3}$ of $\pi_{1}\left(\overline{\mathbb{C}} \backslash\left\{u_{1}, u_{2}, u_{3}, \infty\right\}, \infty\right)$ varies continuously with small variations of $u_{1}, u_{2}, u_{3}$. This new basis is homotopic to the initial one, so we can identify them. 
a full system of first integrals for such equations. We denote $\mathcal{A}\left(u_{1}, u_{2}, u_{3} ; M_{1}, M_{2}, M_{3}\right)$ the solution of the Schlesinger equations locally uniquelly determined by the triple of monodromy matrices $\left(M_{1}, M_{2}, M_{3}\right)$.

All the above arguments remain valid for a general $2 \times 2$ Fuchsian system, provided the non-resonancy condition of the eigenvalues of $\mathcal{A}_{i}$ and $\mathcal{A}_{\infty}$.

Remark 1.2. We observe that the isomonodromy deformations equations preserve the connection matrices $C_{i}$ too. This follows from Lemma 1.1.

1.1.3. Reduction to the PVI $\mu$ equation. Let us now explain, following [JMU], how to reduce the Schlesinger equations (1.14) to the PVI $\mu$ equation. The Schlesinger equations are invariant with respect to the gauge transformations of the form:

$$
\mathcal{A}_{i} \mapsto D^{-1} \mathcal{A}_{i} D, \quad i=1,2,3, \quad \text { for any } D \text { diagonal matrix. }
$$

First of all we have to factor out such gauge transformations; to this aim, we introduce two coordinates $(p, q)$ on the quotient of the space of the matrices satisfying (1.2) with respect to the equivalence relation

$$
\mathcal{A}_{i} \sim D^{-1} \mathcal{A}_{i} D, \quad i=1,2,3, \quad \text { for any } D \text { diagonal matrix. }
$$

The coordinates $(p, q)$ are defined as follows: $q$ is the root of the following linear equation:

$$
\left[\mathcal{A}\left(q ; u_{1}, u_{2}, u_{3}\right)\right]_{12}=0
$$

and $p$ is given by:

$$
p=\left[\mathcal{A}\left(q ; u_{1}, u_{2}, u_{3}\right)\right]_{11},
$$

where $\mathcal{A}\left(z ; u_{1}, u_{2}, u_{3}\right)$ is given in (1.13). The matrices $\mathcal{A}_{i}$ are expressed rationally in terms of the coordinates $(p, q)$ and an auxiliary coordinate $k$, coming from the gauge freedom (1.15):

$$
\begin{aligned}
& \left(\mathcal{A}_{i}\right)_{11}=-\left(\mathcal{A}_{i}\right)_{22}=\frac{q-u_{i}}{2 \mu P^{\prime}\left(u_{i}\right)}\left[P(q) p^{2}+2 \mu \frac{P(q)}{q-u_{i}} p+\mu^{2}\left(q+2 u_{i}-\sum_{j} u_{j}\right)\right], \\
& \left(\mathcal{A}_{i}\right)_{12}=-\mu k \frac{q-u_{i}}{P^{\prime}\left(u_{i}\right)} \\
& \left(\mathcal{A}_{i}\right)_{21}=k^{-1} \frac{q-u_{i}}{4 \mu^{3} P^{\prime}\left(u_{i}\right)}\left[P(q) p^{2}+2 \mu \frac{P(q)}{q-u_{i}} p+\mu^{2}\left(q+2 u_{i}-\sum_{j} u_{j}\right)\right]^{2},
\end{aligned}
$$

for $i=1,2,3$, where $P(z)=\left(z-u_{1}\right)\left(z-u_{2}\right)\left(z-u_{3}\right)$ and $P^{\prime}(z)=\frac{\mathrm{d} P}{\mathrm{~d} z}$. The Schlesinger equations in these coordinates reduce to:

$$
\begin{aligned}
\frac{\partial q}{\partial u_{i}} & =\frac{P(q)}{P^{\prime}\left(u_{i}\right)}\left[2 p+\frac{1}{q-u_{i}}\right] \\
\frac{\partial p}{\partial u_{i}} & =-\frac{P^{\prime}(q) p^{2}+\left(2 q+u_{i}-\sum_{j} u_{j}\right) p+\mu(1-\mu)}{P^{\prime}\left(u_{i}\right)}
\end{aligned}
$$


for $i=1,2,3$. The system of the reduced Schlesinger equations (1.17) is invariant under the transformations of the form

$$
u_{i} \mapsto a u_{i}+b, \quad q \mapsto a q+b, \quad p \mapsto \frac{p}{a}, \quad \forall a, b \in \mathbb{C}, \quad a \neq 0 .
$$

We introduce the following new invariant variables:

$$
\begin{aligned}
& x=\frac{u_{2}-u_{1}}{u_{3}-u_{1}}, \\
& y=\frac{q-u_{1}}{u_{3}-u_{1}} ;
\end{aligned}
$$

the system (1.17), expressed in the these new variables, reduces to the PVI $\mu$ equation for $y(x)$.

Remark 1.3. The system (1.17) admits the following singular solutions (see [Ok1] and [Wat]):

$$
q \equiv u_{i} \quad \text { for some } i,
$$

and $p$, in the variable $x$, can be expressed via Gauss hypergeometric functions (see [Ok1]). Moreover the monodromy group of the system (1.1) reduces to the monodromy group of the Gauss hypergeometric equation, namely the following lemma holds true:

Lemma 1.3. The solutions of the full Schlesinger equations, corresponding to the solution $q \equiv u_{i}$, for some $i$, have the form:

$$
A_{i}(u) \equiv 0, \quad \text { and for } j \neq i \quad A_{j}(u)=D(u)^{-1} A_{j}^{0} D(u),
$$

where $D(u)$ is a diagonal matrix depending on $u$, and $A_{j}^{0}$ is a constant matrix. The monodromy matrix $M_{i}$ of the corresponding Fuchsian system turns out to be the identity. Conversely, if one of the monodromy matrices $M_{i}$ is the identity, $M_{i}=\mathbf{1}$, then the solution of (1.17) is degenerate.

Proof. The matrix $A_{i}$, for $q \equiv u_{i}$, is identically 0 , thanks to (1.16). Having $A_{i} \equiv 0, M_{i}$ is 1. Conversely, if $M_{i}=\mathbf{1}$, then $A_{i} \equiv 0$. Solving the Schlesinger equations (1.13), we obtain $q \equiv u_{i}$, and the equation for $p$ is reduced to a Gauss hypergeometric equation.

QED

The singular solutions do not give any solution of the PVI $\mu$ equation. All the other solutions do, via (1.18). Conversely, starting from any solution $y(x)$ of $\mathrm{PVI} \mu$, we arrive at the solution:

$$
\begin{aligned}
& q=\left(u_{3}-u_{1}\right) y\left(\frac{u_{2}-u_{1}}{u_{3}-u_{1}}\right)+u_{1} \\
& p=\frac{P^{\prime}\left(u_{2}\right)}{2 P(q)} y^{\prime}\left(\frac{u_{2}-u_{1}}{u_{3}-u_{1}}\right)-\frac{1}{2} \frac{1}{q-u_{2}}
\end{aligned}
$$

of the reduced Schlesinger equations (1.17). To obtain a solution of the full Schlesinger equations, the function $k$ must be given by a quadrature:

$$
\frac{\partial k}{\partial u_{i}}=(2 \mu-1) \frac{q-u_{i}}{P^{\prime}\left(u_{i}\right)} .
$$

We conclude this section summarizing all the above results in the following: 
Theorem 1.3. The branches of solutions of the PVI $\mu$ equation near a given point $x_{0} \in$ $\overline{\mathbb{C}} \backslash\{0,1, \infty\}$, are in one-to-one correspondence with the triples of the monodromy matrices $M_{1}, M_{2}, M_{3}$ satisfying (1.7) and (1.8), with $M_{\infty}$ of the form (1.4), none of them being equal to $\mathbf{1}$, considered modulo diagonal conjugations.

Remark 1.4. A triple of $2 \times 2$ matrices $M_{1}, M_{2}, M_{3} \in S L(2 ; \mathbb{C})$, considered modulo conjugations, is a point $\rho$ of the space of representations

$$
\rho: F_{3} \rightarrow S L(2 ; \mathbb{C})
$$

of the free group $F_{3}$ with three generators $\gamma_{1}, \gamma_{2}, \gamma_{3}$, specified by

$$
M_{i}=\rho\left(\gamma_{i}\right), \quad i=1,2,3 .
$$

In the general case, i.e. with the matrices $\mathcal{A}_{i}$ and $\mathcal{A}_{\infty}$ not necassarly of the form (1.2), the corresponding solution $(p, q)$ of the reduced Schlesinger equations will be denoted

$$
p=p\left(u_{1}, u_{2}, u_{3} ; \rho\right), \quad q=q\left(u_{1}, u_{2}, u_{3} ; \rho\right) .
$$

It is locally uniquelly specified by the representation $\rho$, provided the non-resonancy condition of the eigenvalues of $\mathcal{A}_{i}$ and $\mathcal{A}_{\infty}$.

\subsection{The structure of the analytic continuation.}

We parameterized branches of the solutions of PVI $\mu$ by triples of monodromy matrices. Now we show how do these parameters change with a change of the branch in the process of analytic continuation of the solutions along a path in $\overline{\mathbb{C}} \backslash\{0,1, \infty\}$. Recall that, as it follows from Theorem 1.1, the solutions of PVI $\mu$, defined in a neighborhood of a given point $x_{0} \in \overline{\mathbb{C}} \backslash\{0,1, \infty\}$, can be analytically continued to a meromorphic function on the universal covering of $\overline{\mathbb{C}} \backslash\{0,1, \infty\}$ (the above mentioned Painlevé Property). The fundamental group $\pi_{1}(\overline{\mathbb{C}} \backslash\{0,1, \infty\})$ is non-abelian. As a consequence, the global structure of the analytic continuation of the solutions of PVI is more involved than that of the other Painlevé equations. In fact the solutions of PI,..., PV have at most two critical singularities and the corresponding fundamental group is abelian.

As a first step we introduce a parameterization of the monodromy matrices.

1.2.1. The parameterization of the monodromy data Let $\mathcal{M}_{1}, \mathcal{M}_{2}$ and $\mathcal{M}_{3}$ be three linear operators $\mathcal{M}_{i}: \mathbb{C}^{2} \rightarrow \mathbb{C}^{2}$ satisfying (1.7). We introduce for them a parameterization which will be useful for studying the analytic continuation of the solutions of the PVI $\mu$ equation.

Lemma 1.4. If $\mathcal{M}_{1}, \mathcal{M}_{2}$ are such that

$$
\operatorname{Tr}\left(\mathcal{M}_{1} \mathcal{M}_{2}\right) \neq 2,
$$

then there exists a basis in $\mathbb{C}^{2}$ such that, in this basis, the matrices of $\mathcal{M}_{1}, \mathcal{M}_{2}$ have the form:

$$
M_{1}=\left(\begin{array}{cc}
1 & -x_{1} \\
0 & 1
\end{array}\right), \quad M_{2}=\left(\begin{array}{cc}
1 & 0 \\
x_{1} & 1
\end{array}\right),
$$


where $x_{1}=\sqrt{2-\operatorname{Tr}\left(\mathcal{M}_{1} \mathcal{M}_{2}\right)}$; when $\mathcal{M}_{1}, \mathcal{M}_{2}$ are such that $\operatorname{Tr}\left(\mathcal{M}_{1} \mathcal{M}_{2}\right)=2$, they have a common eigenvector, and then there exists a basis in $\mathbb{C}^{2}$ such that, in this basis, the matrices $M_{1}, M_{2}$ are both upper-triangular.

Proof. Due to the (1.7), there exist two vectors $e_{1}$ and $e_{2}$ such that

$$
\mathcal{M}_{1} e_{1}=e_{1}, \quad \mathcal{M}_{2} e_{2}=e_{2}
$$

We now prove that these two vectors are linearly dependent if and only if $\operatorname{Tr}\left(\mathcal{M}_{1} \mathcal{M}_{2}\right)=2$. In fact if the two vectors are linearly dependent, then we can find a linear independent vector $e_{2}^{\prime}$ such that, in the basis $\left(e_{1}, e_{2}^{\prime}\right)$ the matrices of $M_{1}, M_{2}$ have the form:

$$
M_{1}=\left(\begin{array}{cc}
1 & \lambda_{1} \\
0 & 1
\end{array}\right), \quad M_{2}=\left(\begin{array}{cc}
1 & \lambda_{2} \\
0 & 1
\end{array}\right)
$$

so $\operatorname{Tr}\left(M_{1} M_{2}\right)=2$. Conversely, in the basis $\left(e_{1}, e_{2}^{\prime}\right)$ the matrix $M_{1}$ has the form $M_{1}=$ $\left(\begin{array}{cc}1 & \lambda_{1} \\ 0 & 1\end{array}\right)$ and, requiring that

$$
\operatorname{Tr}\left(\mathcal{M}_{1} \mathcal{M}_{2}\right)=2, \quad \operatorname{eigenv}\left(M_{2}\right)=1
$$

also the matrix $M_{2}$ must have the above form $M_{2}=\left(\begin{array}{cc}1 & \lambda_{2} \\ 0 & 1\end{array}\right)$. Then, the two vectors $e_{1}$ and $e_{2}$ are linearly dependent. As a consequence, if $\operatorname{Tr}\left(\mathcal{M}_{1} \mathcal{M}_{2}\right) \neq 2$, the two vectors $e_{1}$ and $e_{2}$ are linearly independent, and in the basis $\left(e_{1}, e_{2}\right)$ the matrices of $M_{1}, M_{2}$ have the form:

$$
M_{1}=\left(\begin{array}{cc}
1 & \lambda_{1} \\
0 & 1
\end{array}\right), \quad M_{2}=\left(\begin{array}{cc}
1 & 0 \\
\lambda_{2} & 1
\end{array}\right)
$$

with $\operatorname{Tr}\left(M_{1} M_{2}\right)=2+\lambda_{1} \lambda_{2}$. Rescaling the basic vectors $\left(e_{1}, e_{2}\right)$, we obtain the (1.19). QED

Lemma 1.5. Let $\mathcal{M}_{1}, \mathcal{M}_{2}, \mathcal{M}_{3}$ satisfy also the condition (1.8) with $\mathcal{M}_{\infty}$ given by (1.4), and $2 \mu \notin \mathbb{Z}$. Then the following statements are true:

i) If two of the following numbers

$$
\operatorname{Tr}\left(\mathcal{M}_{1} \mathcal{M}_{2}\right), \quad \operatorname{Tr}\left(\mathcal{M}_{1} \mathcal{M}_{3}\right), \quad \operatorname{Tr}\left(\mathcal{M}_{3} \mathcal{M}_{2}\right)
$$

are equal to 2 , then one of the matrices of $M_{i}$ is equal to one.

ii) If $\operatorname{Tr}\left(\mathcal{M}_{1} \mathcal{M}_{2}\right) \neq 2$, then there exists a basis in $\mathbb{C}^{2}$ such that, in this basis, the matrices $M_{1}, M_{2}$ and $M_{3}$ have the form

$$
M_{1}=\left(\begin{array}{cc}
1 & -x_{1} \\
0 & 1
\end{array}\right), \quad M_{2}=\left(\begin{array}{cc}
1 & 0 \\
x_{1} & 1
\end{array}\right), \quad M_{3}=\left(\begin{array}{cc}
1+\frac{x_{2} x_{3}}{x_{1}} & -\frac{x_{2}^{2}}{x_{1}} \\
\frac{x_{3}^{2}}{x_{1}} & 1-\frac{x_{2} x_{3}}{x_{1}}
\end{array}\right)
$$

where

$$
\operatorname{Tr}\left(M_{1} M_{2}\right)=2-x_{1}^{2}, \quad \operatorname{Tr}\left(M_{3} M_{2}\right)=2-x_{2}^{2}, \quad \operatorname{Tr}\left(M_{1} M_{3}\right)=2-x_{3}^{2},
$$


and

$$
x_{1}^{2}+x_{2}^{2}+x_{3}^{2}-x_{1} x_{2} x_{3}=4 \sin ^{2} \pi \mu .
$$

iii) If two triples of matrices $M_{1}, M_{2}, M_{3}$ and $M_{1}^{\prime}, M_{2}^{\prime}, M_{3}^{\prime}$ satisfying (1.8), with none of them equal to $\mathbf{1}$, have the form (1.20) with the parameters $\left(x_{1}, x_{2}, x_{3}\right)$ and $\left(x_{1}^{\prime}, x_{2}^{\prime}, x_{3}^{\prime}\right)$ respectively, then these triples are conjugated

$$
M_{i}=T^{-1} M_{i}^{\prime} T
$$

with some invertible matrix $T$ if and only if the triple $\left(x_{1}^{\prime}, x_{2}^{\prime}, x_{3}^{\prime}\right)$ is equal to the triple $\left(x_{1}, x_{2}, x_{3}\right)$, up to the change of the sign of two of the coordinates.

Proof.

i) Let us assume that

$$
\operatorname{Tr}\left(\mathcal{M}_{1} \mathcal{M}_{2}\right)=2, \quad \operatorname{Tr}\left(\mathcal{M}_{1} \mathcal{M}_{3}\right)=2 .
$$

Let $e_{1}$ and $e_{3}$ be the common eigenvectors of $\mathcal{M}_{1}, \mathcal{M}_{2}$ and $\mathcal{M}_{1}, \mathcal{M}_{3}$ respectively, (see Lemma 1.4). If $\mathcal{M}_{1} \neq \mathbf{1}$, then the eigenvectors $e_{1}$ and $e_{3}$ coincide. Then we can find a linear independent vector $e_{2}^{\prime}$ such that, in the basis $\left(e_{1}, e_{2}^{\prime}\right)$ the matrices of $M_{1}, M_{2}$, $M_{3}$ all have the form

$$
M_{i}=\left(\begin{array}{cc}
1 & \lambda_{i} \\
0 & 1
\end{array}\right), \quad i=1,2,3
$$

Then

$$
\operatorname{Tr}\left(M_{3} M_{2} M_{1}\right) 1 \operatorname{Tr}\left(M_{\infty}\right)=2 .
$$

This contradicts the assumption $2 \mu \notin \mathbb{Z}$.

ii) Let us choose the basis such that, according to Lemma 1.4, the matrices $M_{1}, M_{2}$ have the form (1.19). Solving the equations

$$
\operatorname{Tr}\left(M_{3} M_{2}\right)=2-x_{2}^{2}, \quad \operatorname{Tr}\left(M_{1} M_{3}\right)=2-x_{3}^{2},
$$

we arrive at the formula (1.20). The (1.21) is obtained by straightforward computations from

$$
\operatorname{Tr}\left(M_{3} M_{2} M_{1}\right)=2 \cos 2 \pi \mu .
$$

iii) The two triples of matrices $M_{1}, M_{2}, M_{3}$ and $M_{1}^{\prime}, M_{2}^{\prime}, M_{3}^{\prime}$ are conjugated

$$
M_{i}=T^{-1} M_{i}^{\prime} T
$$

with some invertible matrix $T$ if and only if they are the matrices of the same operators $\mathcal{M}_{1}, \mathcal{M}_{2}, \mathcal{M}_{3}$, written in different bases. Since the traces do not depend on the choice of the basis, then

$$
x_{i}^{2}=x_{i}^{\prime 2}, \quad i=1,2,3 .
$$

According to the proof of Lemma 1.4, the basis $\left(e_{1}, e_{2}\right)$ is uniquely determined up to changes of sign. A change of sign $e_{1} \mapsto-e_{1}$ corresponds to the change of sign $x_{1} \mapsto-x_{1}$; then the form of the matrix $M_{3}$ is preserved if and only if we change one of the signs of $x_{2}$ or $x_{3}$.

QED 
Remark 1.5. The matrices (1.20) have a simple geometrical meaning. Let us consider the three-dimensional linear space with a basis $\left(e_{1}, e_{2}, e_{3}\right)$ and with a skew-symmetric bilinear form $\{\cdot, \cdot\}$ such that

$$
\left\{e_{1}, e_{2}\right\}=x_{1}, \quad\left\{e_{1}, e_{3}\right\}=x_{3}, \quad\left\{e_{2}, e_{3}\right\}=x_{2} .
$$

Let us consider the reflections $R_{1}, R_{2}, R_{3}$ in this space, with respect to the hyperplanes skew-orthogonal to the basic vectors:

$$
R_{i}(x)=x-\left\{e_{i}, x\right\} e_{i}, \quad i=1,2,3 .
$$

The reflections have a one-dimensional invariant subspace, namely the kernel of the bilinear form. The matrices of the reflections acting on the quotient are the (1.20).

Definition. A triple $\left(x_{1}, x_{2}, x_{3}\right)$ is called admissible if it has at most one coordinate equal to zero. Two such triples are called equivalent if they are equal up to the change of two signs of the coordinates.

Observe that for an admissible triple $\left(x_{1}, x_{2}, x_{3}\right)$ none of the matrices (1.20) is equal to the identity. So the admissible triples correspond to the non-singular solutions of the reduced Schlesinger equations (1.17). Moreover, two equivalent triples generate the same solution. We can summarize the above results in the following:

Theorem 1.4. The branches of solutions of the PVI $\mu$ equation near a given point $x_{0} \in$ $\overline{\mathbb{C}} \backslash\{0,1, \infty\}$ are in one-to-one correspondence with the equivalence classes of the admissible triples satisfying (1.21).

Proof. Starting from a solution of PVI $\mu$ we obtain the monodromy matrices satisfying (1.7). None of them is equal to the identity. So the canonical form (1.20) of $M_{1}, M_{2}, M_{3}$ is determined uniquely up to a choice of the admissible triple $\left(x_{1}, x_{2}, x_{3}\right)$ within the equivalence class. Conversely, given an admissible triple $\left(x_{1}, x_{2}, x_{3}\right)$ satisfying (1.21), we obtain the matrices $M_{1}, M_{2}, M_{3}$ of the form (1.20). The matrix $M_{3} M_{2} M_{1}$ is diagonalizable with the eigenvalues $\exp ( \pm 2 \pi i \mu$ ) (here we use the non-resonance condition $2 \mu \notin \mathbb{Z}$ ). Reducing this matrix to the diagonal form

$$
M_{3} M_{2} M_{1}=T^{-1}\left(\begin{array}{cc}
\exp (2 \pi i \mu) & 0 \\
0 & \exp (-2 \pi i \mu)
\end{array}\right) T
$$

we obtain the monodromy matrices $T M_{i} T^{-1}$ satisfying (1.7) and thus specifying a branch of the solution of PVI $\mu$.

1.2.2. Monodromy data and symmetries of $\mathbf{P V I} \mu$. The Painlevé VI equation possesses a rich family of symmetries, i. e. transformations of the dependent and independent variables $(y, x)$, and also of the parameters, that preserve the shape of the equation. The theory of these symmetries, and its applications to the construction of particular solutions, was developed in $[\mathrm{Ok}]$. Here we list the symmetries which preserve our PVI $\mu$ and compute their action on the monodromy data. 
First of all we observe that the trivial symmetry $\mu \mapsto 1-\mu$ preserves the Painleve' equation, i.e. PVI $\mu=\operatorname{PVI}(1-\mu)$, so it maps the solutions $y(x)$ in themselves.

Then we consider the permutations of the poles $u_{1}, u_{2}, u_{3}$ which generate the action of the symmetric group $S_{3}$ on the solutions $y(x)$. In particular the involution

$$
i_{1}: u_{2} \leftrightarrow u_{3}
$$

produces the transformation

$$
x \mapsto \frac{1}{x}, \quad y \mapsto \frac{y}{x}
$$

and

$$
i_{2}: u_{1} \leftrightarrow u_{3}
$$

produces the transformation

$$
x \mapsto 1-x, \quad y \mapsto 1-y
$$

Both these transformations clearly preserve the equation $\operatorname{PVI} \mu$.

Let us compute the action of these symmetries on the monodromy data. The only thing that changes is the basis in the fundamental group $\pi_{1}\left(\overline{\mathbb{C}} \backslash\left\{u_{1}, u_{2}, u_{3}, \infty\right\}\right)$. In fact, the cuts $\pi_{1}, \pi_{2}, \pi_{3}$ along which we take our basis $\gamma_{1}, \gamma_{2}, \gamma_{3}$, are ordered according to the order of the poles. Applying the transformation $i_{1}$ we then arrive at the new basis $\gamma_{1}^{\prime}, \gamma_{2}^{\prime}, \gamma_{3}^{\prime}$ shown in figure 2.

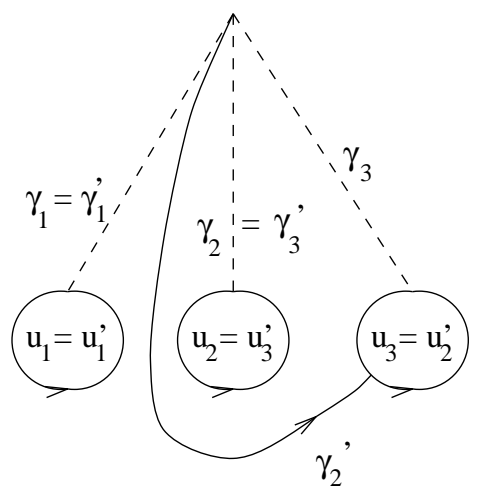

Fig.2. The new basis $\gamma_{1}^{\prime}, \gamma_{2}^{\prime}, \gamma_{3}^{\prime}$ obtained by the action of $i_{1}$.

This new basis has the following form

$$
\gamma_{1}^{\prime}=\gamma_{1}, \quad \gamma_{2}^{\prime}=\gamma_{2} \gamma_{3} \gamma_{2}^{-1}, \quad \gamma_{3}^{\prime}=\gamma_{2}
$$

As a consequence the new monodromy matrices are

$$
M_{1}^{\prime}=M_{1}, \quad M_{2}^{\prime}=M_{2}^{-1} M_{3} M_{2}, \quad M_{3}^{\prime}=M_{2}
$$




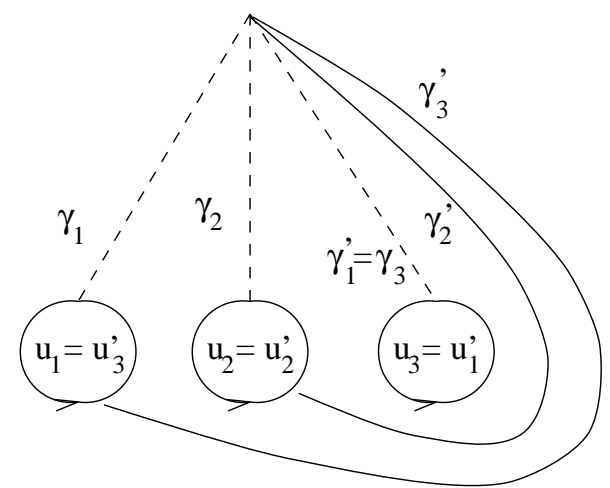

Fig.3. The new basis $\gamma_{1}^{\prime}, \gamma_{2}^{\prime}, \gamma_{3}^{\prime}$ obtained by the action of $i_{2}$.

For the second transformation $i_{2}$, the basis of the new loops is shown in figure 3 . It has the following form

$$
\gamma_{1}^{\prime}=\gamma_{3}, \quad \gamma_{2}^{\prime}=\gamma_{3}^{-1} \gamma_{2} \gamma_{3}, \quad \gamma_{3}^{\prime}=\gamma_{3}^{-1} \gamma_{2}^{-1} \gamma_{1} \gamma_{2} \gamma_{3}
$$

The new monodromy matrices are

$$
M_{1}^{\prime}=M_{3}, \quad M_{2}^{\prime}=M_{3} M_{2}^{-1} M_{3}, \quad M_{3}^{\prime}=M_{3} M_{2} M_{1} M_{2}^{-1} M_{3}^{-1} .
$$

Lemma 1.6. In the coordinates $\left(x_{1}, x_{2}, x_{3}\right)$ on the space of the monodromy matrices, the action of the symmetries $i_{1}, i_{2}$ is given by the formulae

$$
i_{1}:\left(x_{1}, x_{2}, x_{3}\right) \mapsto\left(x_{3}-x_{1} x_{2},-x_{2}, x_{1}\right), \quad i_{2}:\left(x_{1}, x_{2}, x_{3}\right) \mapsto\left(-x_{2},-x_{1}, x_{1} x_{2}-x_{3}\right)
$$

The proof is straightforward.

The last symmetry is more complicated because it changes the value of the parameter $\mu$, i.e. $\mu \mapsto-\mu$, or equivalently $\mu \mapsto 1+\mu$ as it follows form the fact that $\mathrm{PVI}(-\mu)=$ $\operatorname{PVI}(1+\mu)$. This simmetry comes from the following simultaneous conjugation of the coefficients of the Fuchsian system:

$$
A_{i} \rightarrow \Sigma A_{i} \Sigma
$$

where

$$
\Sigma=\Sigma^{-1}=\left(\begin{array}{ll}
0 & 1 \\
1 & 0
\end{array}\right)
$$

Indeed,

$$
\Sigma A_{\infty} \Sigma=-A_{\infty}
$$

Using the parameterization (1.16) of the matrices $A_{1}, A_{2}, A_{3}$ by the coordinates $(p, q)$, we arrive at the following 
Lemma 1.7. The formula

$$
\tilde{y}=y \frac{\left(p_{0}\left(y^{\prime}\right)^{2}+p_{1} y^{\prime}+p_{2}\right)^{2}}{q_{0}\left(y^{\prime}\right)^{4}+q_{1}\left(y^{\prime}\right)^{3}+q_{2}\left(y^{\prime}\right)^{2}+q_{3} y^{\prime}+q_{4}},
$$

where

$$
\begin{aligned}
p_{0} & =x^{2}(x-1)^{2} \\
p_{1} & =2 x(x-1)(y-1)[2 \mu(y-x)-y] \\
p_{2} & =y(y-1)\left[y(y-1)-4 \mu(y-1)(y-x)+4 \mu^{2}(y-x)(y-x-1)\right] \\
q_{0} & =x^{4}(x-1)^{4} \\
q_{1} & =-4 x^{3}(x-1)^{3} y(y-1) \\
q_{2} & =2 x^{2}(x-1)^{2} y(y-1)\left[3 y(y-1)+4 \mu^{2}(y-x)(1+x-3 y)\right] \\
q_{3} & =4 x(x-1) y^{2}(y-1)^{2}\left[-y(y-1)-16 \mu^{3}(y-x)^{2}+4 \mu^{2}(y-x)(3 y-x-1)\right] \\
q_{4} & =y^{2}(y-1)^{2}\left\{y^{2}(y-1)^{2}+64 \mu^{3} y(y-1)(y-x)^{2}-8 \mu^{2} y(y-1)(y-x)(3 y-x-1)+\right. \\
& \left.+16 \mu^{4}(y-x)^{2}\left[(x-1)^{2}+y(2+2 x-3 y)\right]\right\}
\end{aligned}
$$

transforms solutions of PVI $\mu$ to solutions of PVI $(-\mu)$. The class of equivalence of the monodromy data $\left(x_{1}, x_{2}, x_{3}\right)$ does not change under such a symmetry.

Proof. The new monodromy matrices $M_{1}^{\prime}, M_{2}^{\prime}, M_{3}^{\prime}$ have the form

$$
M_{i}^{\prime}=\Sigma M_{i} \Sigma, \quad i=1,2,3 .
$$

Then, the canonical form (1.20) of the monodromy operators does not change.

QED

Other symmetries are superpositions of (1.24) with the trivial one $\mu \rightarrow 1-\mu$. Using these symmetries, one can transform $\mathrm{PVI}_{\mu}$ to $\mathrm{PVI}_{\mu^{\prime}}$, with $\mu^{\prime}= \pm \mu+n$ for an arbitrary integer $n$.

Remark 1.6. One can show that the above symmetries, and their superpositions, exhaust all the birational transformations preserving our one-parameter family of PVI equations. We will not do it here (see $[\mathrm{Ok}]$ ). It is important, however, that these symmetries preserve the class of algebraic solutions of $\mathrm{PVI} \mu$. We will classify all the algebraic solutions modulo the above symmetries.

Remark 1.7. It is not difficult to show that the denominator of the formula (1.24) does not vanish identically for any solution of $\mathrm{PVI} \mu$, with $2 \mu \notin \mathbb{Z}$. Indeed, eliminating $y_{x x}$ and $y_{x}$ form the system

$$
\begin{aligned}
y_{x x}= & \frac{1}{2}\left(\frac{1}{y}+\frac{1}{y-1}+\frac{1}{y-x}\right) y_{x}^{2}-\left(\frac{1}{x}+\frac{1}{x-1}+\frac{1}{y-x}\right) y_{x} \\
& +\frac{1}{2} \frac{y(y-1)(y-x)}{x^{2}(x-1)^{2}}\left[(2 \mu-1)^{2}+\frac{x(x-1)}{(y-x)^{2}}\right]
\end{aligned}
$$




$$
\begin{aligned}
Q\left(y_{x}, y, x, \mu\right) & =0, \\
\frac{\mathrm{d}}{\mathrm{d} x} Q\left(y_{x}, y, x, \mu\right) & =0,
\end{aligned}
$$

where $Q$ is the denominator, the resultant equation

$$
(2 \mu+1)^{4} \mu^{16}\left[x(x-1)^{2}\right]^{4}[y(y-1)(y-x)]^{4}
$$

never vanishes.

\subsubsection{The analytic continuation of the solutions of PVI $\mu$ and the braid group} $B_{3}$. In this subsection, we describe the procedure of the analytic continuation towards an action of the braid group on the admissible triples $\left(x_{1}, x_{2}, x_{3}\right)$ parameterizing the branches of the solutions of PVI $\mu$.

According to Theorem 1.1, any solution of the Schlesinger equations can be continued analytically from a point $\left(u_{1}^{0}, u_{2}^{0}, u_{3}^{0}\right)$ to another point $\left(u_{1}^{1}, u_{2}^{1}, u_{3}^{1}\right)$ along a path

$$
\left(u_{1}(t), u_{2}(t), u_{3}(t)\right) \in \mathbb{C}^{3} \backslash\{\text { diags }\}, \quad 0 \leq t \leq 1,
$$

with

$$
u_{i}(0)=u_{i}^{0}, \quad \text { and } \quad u_{i}(1)=u_{i}^{1},
$$

provided that the end-points are not the poles of the solution. The result of the analytic continuation depends only on the homotopy class of the path in $\mathbb{C}^{3} \backslash\{$ diags $\}$. Particularly, to find all the branches of a solution near a given point $u^{0}=\left(u_{1}^{0}, u_{2}^{0}, u_{3}^{0}\right)$ one has to compute the results of the analytic continuation along any homotopy class of closed loops in $\mathbb{C}^{3} \backslash\{$ diags $\}$ with the beginning and the end at the point $u^{0}=\left(u_{1}^{0}, u_{2}^{0}, u_{3}^{0}\right)$. Let

$$
\beta \in \pi_{1}\left(\mathbb{C}^{3} \backslash\{\text { diags }\} ; u^{0}\right)
$$

be an arbitrary loop. Any solution of the Schlesinger equations near the point $u^{0}=$ $\left(u_{1}^{0}, u_{2}^{0}, u_{3}^{0}\right)$, is uniquely determined by the monodromy matrices $M_{1}, M_{2}$ and $M_{3}$, computed in the basis $\gamma_{1}, \gamma_{2}, \gamma_{3}$. Continuing analytically this solution along the loop $\beta$, we arrive at another branch of the same solution near $u^{0}$. This new branch is specified, according to Theorem 1.3, by some new monodromy matrices $M_{1}^{\beta}, M_{2}^{\beta}$ and $M_{3}^{\beta}$, computed in the same basis $\gamma_{1}, \gamma_{2}, \gamma_{3}$. Our nearest goal is to compute these new matrices for any loop $\beta \in \pi_{1}\left(\mathbb{C}^{3} \backslash\{\right.$ diags $\left.\} ; u^{0}\right)$.

The fundamental group $\pi_{1}\left(\mathbb{C}^{3} \backslash\{\right.$ diags $\left.\} ; u^{0}\right)$ is isomorphic to the pure (or unpermuted) braid group, $P_{3}$ with three strings (see [Bir]); this is a subgroup of the full braid group $B_{3}$. The full braid group is isomorphic to the fundamental group of the same space where the permutations are allowed:

$$
B_{3} \simeq \pi_{1}\left(\mathbb{C}^{3} \backslash\{\text { diags }\} / S_{3} ; u^{0}\right)
$$

$S_{3}$ being the symmetric group acting by permutations of the coordinates $\left(u_{1}, u_{2}, u_{3}\right)$. Any loop in $B_{3}$ has the form

$$
\left(u_{1}(t), u_{2}(t), u_{3}(t)\right) \in \mathbb{C}^{3} \backslash\{\text { diags }\}, \quad 0 \leq t \leq 1,
$$


with

$$
u_{i}(0)=u_{i}^{0}, \quad u_{i}(1)=u_{p(i)}^{0},
$$

where $p$ is a permutation of $\{1,2,3\}$. The elements of the subgroup $P_{3}$ of pure braids are specified by the condition $p=$ id.

To simplify the computations we extend the procedure of the analytic continuation to the full braid group

$$
M_{1}, M_{2}, M_{3} \mapsto M_{1}^{\beta}, M_{2}^{\beta}, M_{3}^{\beta}, \quad \beta \in B_{3}=\pi_{1}\left(\mathbb{C}^{3} \backslash\{\text { diags }\} / S_{3} ; u^{0}\right) .
$$

For a generic braid $\beta \in B_{3}$, the new monodromy matrices describe the superposition of the analytic continuation and of the permutation

$$
u_{i} \mapsto u_{p(i)}, \quad \mathcal{A}_{i} \mapsto \mathcal{A}_{p(i)}
$$

The braid group $B_{3}$ admits a presentation with generators $\beta_{1}$ and $\beta_{2}$ and the defining relation

$$
\beta_{1} \beta_{2} \beta_{1}=\beta_{2} \beta_{1} \beta_{2}
$$

The generators $\beta_{1}$ and $\beta_{2}$ are shown in the figure 4 .
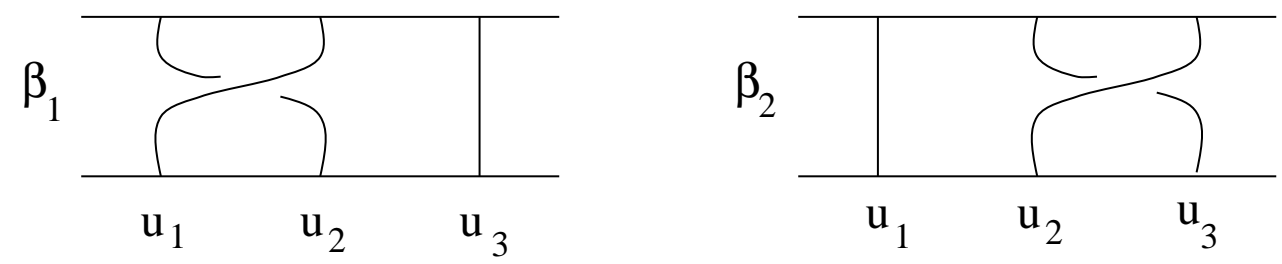

Fig.4. The generators of the braid group $B_{3}$.

Lemma 1.8. For the generators $\beta_{1}, \beta_{2}$ shown in the figure 4 , the matrices $M_{i}^{\beta}$ have the following form:

$$
\begin{array}{ll}
M_{1}^{\beta_{1}}=M_{2}, & M_{2}^{\beta_{1}}=M_{2} M_{1} M_{2}^{-1}, \quad M_{3}^{\beta_{1}}=M_{3}, \\
M_{1}^{\beta_{2}}=M_{1}, & M_{2}^{\beta_{2}}=M_{3}, \quad M_{3}^{\beta_{2}}=M_{3} M_{2} M_{3}^{-1} .
\end{array}
$$

Proof. Changing the positions of the points $u_{1}$ and $u_{2}$ by the braid $\beta_{1}$, the basis of the loops will be deformed into the new basis $\gamma_{1}^{\prime}, \gamma_{2}^{\prime}, \gamma_{3}^{\prime}$ shown in the figure 5 .

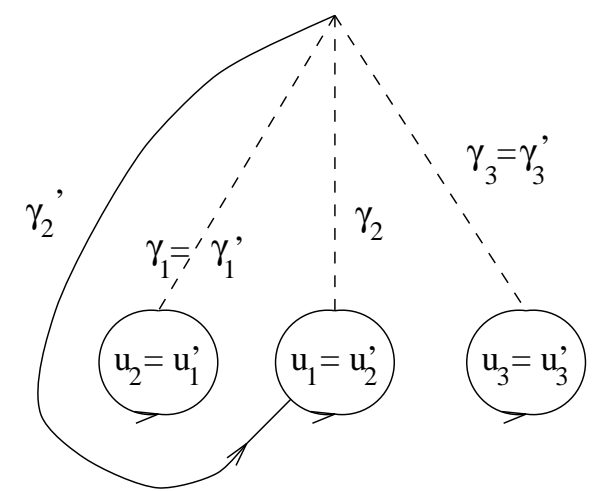

Fig.5. The new loops $\gamma_{i}^{\prime}$ obtained under the action of the braid $\beta_{1}$. 
Thanks to the fact that we deal with isomonodromy deformations, the monodromy matrices $M_{i}^{\prime}$ of the system (1.1) with respect to the new basis $\gamma_{1}^{\prime}, \gamma_{2}^{\prime}, \gamma_{3}^{\prime}$ are the same $M_{i}$, up to the reordering:

$$
M_{1}^{\prime}=M_{2}, \quad M_{2}^{\prime}=M_{1}, \quad M_{3}^{\prime}=M_{3} .
$$

We want to compute the monodromy matrices with respect to the old basis $\gamma_{1}, \gamma_{2}, \gamma_{3}$. To this aim we notice the following obvious relation in the fundamental group:

$$
\gamma_{1}=\gamma_{1}^{\prime}, \quad \gamma_{2}=\left(\gamma_{1}^{\prime}\right)^{-1} \gamma_{2}^{\prime} \gamma_{1}^{\prime}, \quad \gamma_{3}=\gamma_{3}^{\prime} .
$$

Using this relations and the (1.29), we immediately obtain the (1.27). Similarly the deformation of the basis of the fundamental group corresponding to the braid $\beta_{2}$ is shown in the figure 6 .

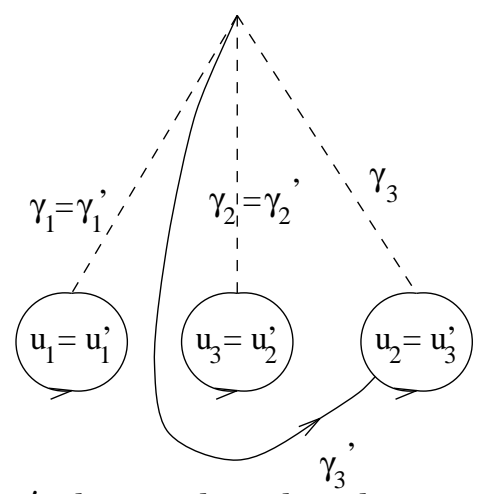

Fig.6. The new loops $\gamma_{i}^{\prime}$ obtained under the action of the braid $\beta_{2}$.

Here we have the permutation

$$
M_{1}^{\prime}=M_{1} \quad M_{2}^{\prime}=M_{3}^{\prime}, \quad M_{3}^{\prime}=M_{2},
$$

and the relations in the fundamental group:

$$
\gamma_{1}=\gamma_{1}^{\prime}, \quad \gamma_{2}=\gamma_{2}^{\prime}, \quad \gamma_{3}=\left(\gamma_{2}^{\prime}\right)^{-1} \gamma_{3}^{\prime} \gamma_{2}^{\prime} .
$$

From this we obtain the (1.28) and the lemma is proved.

QED

The action (1.27), (1.28) of the braid group on the triples of monodromy matrices commutes with the diagonal conjugation of them. As a consequence this action not only describes the structure of the analytic continuation of the solutions of the Schlesinger equations (1.14), but also of the reduced ones (1.17). Moreover, the class of the singular solutions is closed under this analytic continuation. In fact if some of the matrices $M_{i}$ is equal to $\mathbf{1}$ then for any $\beta$ there is a $j$ such that $M_{j}^{\beta}=\mathbf{1}$. As a consequence the following lemma holds true:

Lemma 1.9. The structure of the analytic continuation of the solutions of the PVI $\mu$ equation is determined by the action (1.27), (1.28) of the braid group on the triples of monodromy matrices.

Our next step is to rewrite the action (1.27), (1.28) of the braid group in the coordinates $\left(x_{1}, x_{2}, x_{3}\right)$ in the space of the monodromy data. This is given by the following 
Lemma 1.10. In the coordinates $\left(x_{1}, x_{2}, x_{3}\right)$, the action (1.27), (1.28) of the braid group is given by the formulae:

$$
\begin{aligned}
& \beta_{1}:\left(x_{1}, x_{2}, x_{3}\right) \mapsto\left(-x_{1}, x_{3}-x_{1} x_{2}, x_{2}\right), \\
& \beta_{2}:\left(x_{1}, x_{2}, x_{3}\right) \mapsto\left(x_{3},-x_{2}, x_{1}-x_{2} x_{3}\right) .
\end{aligned}
$$

Proof. The above formulae are obtained by straightforward computations from (1.27), (1.28) by means of the parameterization of the monodromy matrices (1.20).

We can summarize the results of this section in the following:

Theorem 1.5. The structure of the analytic continuation of the solutions of the PVI $\mu$ equation is determined by the action (1.30) of the braid group on the triples $\left(x_{1}, x_{2}, x_{3}\right)$.

Remark 1.8. It is easy to see that the braid $\left(\beta_{1} \beta_{2}\right)^{3}$ acts trivially on the monodromy data. This braid is the generator of the center of $B_{3}$ (see [Bir]). The quotient

$$
B_{3} / \text { center } \simeq P S L(2 ; \mathbb{Z})
$$

coincides with the mapping class group of the complex plane with three punctures [Bir]. Also in the general case, the structure of analytic continuation of solutions of PVI equation is described by the following natural action $\rho \rightarrow \rho^{\beta}$ of the mapping class group on the representation space (see remark 1.4)

$$
\rho^{\beta}(\gamma)=\rho\left(\beta_{\star}^{-1}(\gamma)\right)
$$

where

$$
\begin{gathered}
\gamma \in F_{3} \simeq \pi_{1}\left(\overline{\mathbb{C}} \backslash\left\{u_{1}, u_{2}, u_{3}, \infty\right\}, \infty\right), \\
\beta: \overline{\mathbb{C}} \backslash\left\{u_{1}, u_{2}, u_{3}, \infty\right\} \rightarrow \overline{\mathbb{C}} \backslash\left\{u_{1}, u_{2}, u_{3}, \infty\right\}, \quad \beta(\infty)=\infty
\end{gathered}
$$

is a homeomorphism, and

$$
\rho: F_{3} \rightarrow S L(2 ; \mathbb{C}) .
$$

Our action (1.30) is obtained restricting (1.31) onto the subspace of representations of the form (1.7). The problem of selection of algebraic solutions of Painlevé VI (see below) with generic values of the parameters $\alpha, \beta, \gamma, \delta$ can be reduced to the classification of finite orbits of the action (1.31).

\subsection{Monodromy data and algebraic solutions of the $\operatorname{PVI} \mu$ equation.}

1.3.1. A preliminary discussion on the algebraic solutions of the $\operatorname{PVI} \mu$ equation and their monodromy data. Here we state some necessary condition for the triples $\left(x_{1}, x_{2}, x_{3}\right)$ to generate the algebraic solutions.

Definition. A solution $y(x)$ is called algebraic if there exists a polynomial in two variables such that

$$
F(x, y(x)) \equiv 0 .
$$


If $y(x)$ is an algebraic solution then the correspondent solution $p(\mathbf{u}), q(\mathbf{u}), \mathbf{u}=$ $\left(u_{1}, u_{2}, u_{3}\right)$ of the reduced Schlesinger equations (1.17) is also algebraic. According to Theorem 1.1, the solutions of the reduced Schlesinger equations (1.17) can ramify only on the diagonals $u_{1}=u_{2}, u_{1}=u_{3}, u_{3}=u_{2}$. Analogously the ramification points of $y(x)$ are allowed to lie only at $0,1, \infty$.

We now characterize the monodromy data such that the correspondent solution of the $\operatorname{PVI} \mu$ equation is algebraic.

Lemma 1.11. A necessary and sufficient condition for a solution of PVI $\mu$ to be algebraic is that the correspondent monodromy matrices, defined modulo diagonal conjugations, have a finite orbit under the action of the braid group (1.27), (1.28).

Proof. By definition, any algebraic function has a finite number of branches. Allowing also the permutations (1.26), we still obtain a finite number of values for $M_{1}^{\beta}, M_{2}^{\beta}$ and $M_{3}^{\beta}$, $\beta \in B_{3}$ up to diagonal conjugations.

QED

Corollary 1.2. An admissible triple $\left(x_{1}, x_{2}, x_{3}\right)$ specifies an algebraic solution of PVI $\mu$, with $2 \mu \notin \mathbb{Z}$, if and only if it satisfies (1.21) and its orbit, under the action (1.30) of the braid group, is finite.

Remark 1.9. We stress that the action (1.30) preserves the relation (1.21).

In this way, the problem of the classification of all the algebraic solutions of the PVI $\mu$ reduces to the problem of the classification of all the finite orbits of the action (1.30) under the braid group in the three dimensional space (see [Dub], appendix F). Here we give a simple necessary condition for a triple $\left(x_{1}, x_{2}, x_{3}\right)$ to belong to a finite orbit.

Lemma 1.12. Let $\left(x_{1}, x_{2}, x_{3}\right)$ be a triple belonging to a finite orbit. Then:

$$
x_{i}=-2 \cos \pi r_{i}, \quad r_{i} \in \mathbf{Q}, \quad 0 \leq r_{i} \leq 1, \quad i=1,2,3 .
$$

Here $\mathbf{Q}$ is the set of rational numbers.

Proof. Let us prove the statement for, say, the coordinate $x_{1}$. Consider the transformation

$$
\beta_{1}^{2}:\left(x_{1}, x_{2}, x_{3}\right) \mapsto\left(x_{1}, x_{2}+x_{1} x_{3}-x_{1}^{2} x_{2}, x_{3}-x_{1} x_{2}\right),
$$

as a linear map on the plane $\left(x_{2}, x_{3}\right)$. This linear map preserves the quadratic form

$$
x_{2}^{2}+x_{3}^{2}-x_{1} x_{2} x_{3} .
$$

If $x_{1}=2$, we put $r_{1}=1$; otherwise we reduce the quadratic form to the principal axes, introducing the new coordinates

$$
\tilde{x}_{2}=\frac{\sqrt{2+x_{1}}}{2}\left(x_{2}-x_{3}\right), \quad \tilde{x}_{3}=\frac{\sqrt{2+x_{1}}}{2}\left(x_{2}+x_{3}\right) .
$$

In these new coordinates the preserved quadratic form becomes a sum of squares and the transformation $\beta_{1}^{2}$ is a rotation by the angle $\pi+2 \alpha$, where $\alpha$ is such that $x_{1}=-2 \cos \alpha$. 
To have a finite orbit of $\left(\tilde{x}_{2}, \tilde{x}_{2}\right)$ under the iterations of $\beta_{1}^{2}$, the angle $\alpha$ must be a rational multiple of $\pi$. In this way the statement for $x_{1}$ is proved. To prove it for $x_{2}$ and $x_{3}$ we have to consider the iterations of $\beta_{2}^{2}$ and $\beta_{2}^{-1} \beta_{1}^{2} \beta_{2}$ respectively.

QED

Remark 1.10. Thanks to the above lemma, for the finite orbits of the braid group, it is equivalent to deal with the triples $\left(x_{1}, x_{2}, x_{3}\right)$, or with the triangles with angles $\left(\pi r_{1}, \pi r_{2}, \pi r_{3}\right)$, with $x_{i}=-2 \cos \pi r_{i}$ and $0 \leq r_{i} \leq 1$ (we may assume, changing if necessary two of the signs, that at most one of the $x_{i}$ is positive). Observe that the quantity

$$
x_{1}^{2}+x_{2}^{2}+x_{3}^{2}-x_{1} x_{2} x_{3}-4
$$

is greater than 0 if and only if the triangle $\left(r_{1}, r_{2}, r_{3}\right)$ is hyperbolic, namely $\sum r_{i}<1$; it is equal to 0 , if and only if the triangle $\left(r_{1}, r_{2}, r_{3}\right)$ is flat, namely $\sum r_{i}=1$, and it is less than 0 if and only if the triangle $\left(r_{1}, r_{2}, r_{3}\right)$ is spherical, namely $\sum r_{i}>1$. Thanks to (1.21), a flat triangle gives a resonant value of $\mu$, and it is thus forbidden.

1.3.2. Classification of the triples $\left(x_{1}, x_{2}, x_{3}\right)$ corresponding to the algebraic solutions. We deal with the classification of all the finite orbits of the triples $\left(x_{1}, x_{2}, x_{3}\right)$ of the form (1.32), with at most ${ }^{3}$ one $r_{i}$ being equal to $\frac{1}{2}$. According to Lemma 1.12, any point of these $B_{3}$-orbits must have the same form (1.32). This condition is crucial in the classification.

Definition. We say that an admissible triple $\left(x_{1}, x_{2}, x_{3}\right)$ is good if for any braid $\beta \in B_{3}$ one has

$$
\beta\left(x_{1}, x_{2}, x_{3}\right)=\left(-2 \cos \pi r_{1}^{\beta},-2 \cos \pi r_{2}^{\beta},-2 \cos \pi r_{3}^{\beta}\right),
$$

with some rational numbers $0 \leq r_{i}^{\beta} \leq 1$.

Theorem 1.6. Any good triple belongs to the orbit of one of the following five

$$
\begin{aligned}
& \left(-2 \cos \frac{\pi}{2},-2 \cos \frac{\pi}{3},-2 \cos \frac{\pi}{3}\right), \\
& \left(-2 \cos \frac{\pi}{2},-2 \cos \frac{\pi}{3},-2 \cos \frac{\pi}{4}\right), \\
& \left(-2 \cos \frac{\pi}{2},-2 \cos \frac{\pi}{3},-2 \cos \frac{\pi}{5}\right), \\
& \left(-2 \cos \frac{\pi}{2},-2 \cos \frac{\pi}{3},-2 \cos \frac{2 \pi}{5}\right), \\
& \left(-2 \cos \frac{\pi}{2},-2 \cos \frac{\pi}{5},-2 \cos \frac{2 \pi}{5}\right) .
\end{aligned}
$$

All these orbits are finite and pairwise distinct. They contain all the permutations of the triples (1.33), (1.34), (1.35), (1.36) and (1.37), and also the triples

$$
\left(2 \cos \frac{\pi}{3}, 2 \cos \frac{\pi}{3}, 2 \cos \frac{\pi}{3}\right),
$$

3 This corresponds to the fact that we deal only with admissible triples. 


$$
\begin{gathered}
\left(-2 \cos \frac{2 \pi}{3},-2 \cos \frac{\pi}{4},-2 \cos \frac{\pi}{4}\right) \\
\left(-2 \cos \frac{2 \pi}{3},-2 \cos \frac{\pi}{5},-2 \cos \frac{\pi}{5}\right) \quad\left(-2 \cos \frac{4 \pi}{5},-2 \cos \frac{4 \pi}{5},-2 \cos \frac{4 \pi}{5}\right), \\
\left(-2 \cos \frac{2 \pi}{3},-2 \cos \frac{2 \pi}{5},-2 \cos \frac{2 \pi}{5}\right), \quad\left(-2 \cos \frac{2 \pi}{5},-2 \cos \frac{2 \pi}{5},-2 \cos \frac{2 \pi}{5}\right), \\
\left(-2 \cos \frac{3 \pi}{5},-2 \cos \frac{\pi}{3},-2 \cos \frac{\pi}{5}\right),\left(-2 \cos \frac{2 \pi}{5},-2 \cos \frac{\pi}{3},-2 \cos \frac{\pi}{3}\right), \\
\left(-2 \cos \frac{2 \pi}{3},-2 \cos \frac{\pi}{3},-2 \cos \frac{\pi}{5}\right),
\end{gathered}
$$

respectively, together with all their permutations.

Corollary 1.3. There are five finite orbits of the action (1.30) of the braid group on the space of the admissible triples $\left(x_{1}, x_{2}, x_{3}\right)$ satisfying

$$
x_{1}^{2}+x_{2}^{2}+x_{3}^{2}-x_{1} x_{2} x_{3} \neq 4
$$

The lengths of the orbits (1.33), (1.34), (1.35), (1.36) and (1.37), are equal to 4, 9, 10, 10 and 18 respectively.

Remark 1.11. The action of the pure braid group $P_{3}$ on the above orbits gives the same orbits for any of them but (1.34). The orbit (1.34), under the action of the pure braid group $P_{3}$, splits into three different orbits of three points. So the $P_{3}$-orbit (1.33) has four points, the three $P_{3}$-orbits (1.34) have three points each, (1.35) and (1.36) have ten points each and (1.37) has eighteen points. These orbits give rise to all the algebraic solutions of the PVI $\mu$ equation, for $\mu$ is given by (1.21). The number of the points of each orbit with respect to the action of $P_{3}$ coincides with the number of the branches of the correspondent algebraic solution.

Proof of Theorem 1.6. The braid group acting on the classes of triples $\left(x_{1}, x_{2}, x_{3}\right)$, is generated by the braid $\beta_{1}$ and by the cyclic permutation:

$$
\left(x_{1}, x_{2}, x_{3}\right) \mapsto\left(x_{3}, x_{1}, x_{2}\right)
$$

As a consequence it suffices to study the operator:

$$
\left(x_{i}, x_{j}, x_{k}\right) \mapsto\left(-x_{i}, x_{j}, x_{k}-x_{i} x_{j}\right)
$$

up to cyclic permutations. This transformation works on the triangles with angles $\pi r_{i}$, $\pi r_{j}, \pi r_{k}$ as follows:

$$
\left(r_{i}, r_{j}, r_{k}\right) \mapsto\left(1-r_{i}, r_{j}, r_{k}^{\prime}\right)
$$

where $r_{k}^{\prime}$ is such that:

$$
\cos \pi r_{k}^{\prime}=\cos \pi r_{k}+2 \cos \pi r_{i} \cos \pi r_{j}
$$


The first step is to classify all the rational triples $\left(r_{i}, r_{j}, r_{k}\right)$ such that $r_{k}^{\prime}$, defined by (1.39) is a rational number, $1>r_{k}^{\prime}>0$, for every choice of $i \neq j \neq k \neq i, i, j, k=1,2,3$. Equivalently we want to classify all the rational solutions of the following equation:

$$
\cos \pi r_{k}+\cos \pi\left(r_{i}+r_{j}\right)+\cos \pi\left(r_{i}-r_{j}\right)+\cos \pi\left(1-r_{k}^{\prime}\right)=0,
$$

or all the rational quadruples $\left(\varphi_{1}, \varphi_{2}, \varphi_{3}, \varphi_{4}\right)$ such that:

$$
\cos 2 \pi \varphi_{1}+\cos 2 \pi \varphi_{2}+\cos 2 \pi \varphi_{3}+\cos 2 \pi \varphi_{4}=0,
$$

where the $\varphi_{i}$ are related with the $r_{i}$ by the following relations:

$$
\varphi_{1}=r_{k} / 2, \quad \varphi_{2}=\frac{r_{i}+r_{j}}{2}, \quad \varphi_{3}=\frac{\left|r_{i}-r_{j}\right|}{2}, \quad \varphi_{4}=\frac{\left|1-r_{k}^{\prime}\right|}{2} .
$$

Such a classification is given by the following:

Lemma 1.13. The only rational solutions $\left(\varphi_{1}, \varphi_{2}, \varphi_{3}, \varphi_{4}\right), 0 \leq \varphi_{i}<1$, considered up to permutations and up to transformations $\varphi_{i} \rightarrow 1-\varphi_{i}$, of the equation (1.40) consist of the following non-trivial solutions:

$$
\begin{aligned}
& \left(\frac{1}{30}, \frac{11}{30}, \frac{2}{5}, \frac{1}{6}\right) \\
& \left(\frac{7}{30}, \frac{17}{30}, \frac{1}{5}, \frac{1}{6}\right) \\
& \left(\frac{1}{7}, \frac{2}{7}, \frac{3}{7}, \frac{1}{6}\right)
\end{aligned}
$$

and of the following "trivial" ones, of three types:

(d): $\cos 2 \pi \varphi_{4}=0$. The solutions obtained in [Cro] have the form

$$
(d .1):\left(\frac{1}{3}, \frac{1}{10}, \frac{3}{10}, \frac{1}{4}\right),(d .2):\left(\varphi, \varphi+\frac{1}{3}, \varphi+\frac{2}{3}, \frac{1}{4}\right),(d .3):\left(\frac{1}{4}, \varphi,\left|\varphi-\frac{1}{2}\right|, \frac{1}{4}\right),
$$

where $\varphi$ is any rational number $0 \leq \varphi<1$.

(e): $\cos 2 \pi \varphi_{4}=1$. The solutions obtained in [Gor] have the form

$$
(e .1):\left(\frac{1}{3}, \frac{1}{4}, \frac{1}{3}, 0\right), \quad(e .2):\left(\frac{1}{2}, \varphi,\left|\varphi-\frac{1}{2}\right|, 0\right), \quad(e .3):\left(\frac{1}{3}, \frac{1}{5}, \frac{2}{5}, 0\right),
$$

where $\varphi$ is any rational number $0 \leq \varphi<1$.

(f): $\cos 2 \pi \varphi_{1}+\cos 2 \pi \varphi_{2}=0, \quad \cos 2 \pi \varphi_{3}+\cos 2 \pi \varphi_{4}=0$. The solutions are obvious

$$
\varphi_{2}=\left|1 / 2-\varphi_{1}\right|, \quad \varphi_{4}=\left|1 / 2-\varphi_{3}\right|,
$$

where $\varphi_{1}, \varphi_{3}$ are two arbitrary rational numbers $0 \leq \varphi_{i}<1$.

Proof. We follow the idea of Gordan [Gor] (see also [Cro]). In this proof we use the same notations as in [Cro], except for the $\varphi_{i}$ which there are called $r_{i}$. Let us recall the notations. 
Let $\varphi_{k}=\frac{n_{k}}{d_{k}}$ where $d_{k}, n_{k}$ are either positive coprime integers, $d_{k}>n_{k}$, or $n_{k}=0$. Let $p$ be the largest prime which is a divisor of $d_{1}, d_{2}, d_{3}$, or $d_{4}$ and let $\delta_{k}, l_{k}, c_{k}, \nu_{k}$ be the integers such that

$$
d_{k}=\delta_{k} p^{l_{k}} \quad \text { and } \quad n_{k}=c_{k} \delta_{k}+\nu_{k} p^{l_{k}}
$$

where $\delta_{k}$ is prime to $p, 0 \leq c_{k}<p^{l_{k}}, c_{k}=0$ if $l_{k}=0$, but otherwise $c_{k}$ is prime to $p$. So

$$
\varphi_{k}=\frac{\nu_{k}}{\delta_{k}}+\frac{c_{k}}{p^{l_{k}}}=f_{k}+\frac{c_{k}}{p^{l_{k}}}
$$

We assume that $l_{1} \geq l_{2} \geq l_{3} \geq l_{4}$ and define the function:

$$
g_{k}(x)=\left\{\begin{array}{cl}
\frac{1}{2}\left[e^{2 \pi i f_{k}} x^{c_{k} p^{l_{1}-l_{k}}}+e^{-2 \pi i f_{k}} x^{p^{l_{1}}-c_{k} p^{l_{1}-l_{k}}}\right] & \text { if } c_{k} \neq 0 \\
\cos 2 \pi \varphi_{k} & \text { if } c_{k}=0
\end{array}\right.
$$

and, in our case:

$$
U(x)=\sum_{1}^{4} g_{k}(x)
$$

As in [Cro], $g_{k}\left(\exp \left(\frac{2 \pi i}{p^{l_{1}}}\right)\right)=\cos 2 \pi \varphi_{k}$ and $U\left(\exp \left(\frac{2 \pi i}{p^{l_{1}}}\right)\right)=0$. Let us introduce the polynomial

$$
P(x)=1+x^{p^{l_{1}-1}}+x^{2 p^{l_{1}-1}} \cdots x^{(p-1) p^{l_{1}-1}} .
$$

This is the minimal polynomial of $\exp \left(\frac{2 \pi i}{p^{l_{1}}}\right)$ with coefficients in $\mathbf{Q}$, that is such that i) $P\left(\exp \left(\frac{2 \pi i}{p^{l_{1}}}\right)\right)=0$ and ii) $P(x)$ is irreducible in the ring of polynomials with rational coefficients. A stronger result was proved by Kronecker (see $[\mathrm{Kr}]$ ): the polynomial $P(x)$ remains irreducible over any extension of the form $\mathbf{Q}\left(\zeta_{1}, \cdots, \zeta_{n}\right)$, where $\zeta_{i}$ is a root of the unity of the order coprime with $p$. As a consequence, the following lemma holds true (see [Gor $]$ )

Lemma 1.14. If we express the polynomial $U(x)$ as a sum of polynomials $U_{t}(x)$,

$$
U(x)=\sum_{t=0}^{p^{l_{1}-1}-1} U_{t}(x)
$$

where $U_{t}(x)$ contains those terms of $U(x)$ of the form $b x^{c}$ with $c=t \bmod \left(p^{l_{1}-1}\right)$, then every $U_{t}(x)$ is divisible by $P(x)$.

We now apply this lemma in our case. The indices of the powers of $x$ are:

$$
c_{1}, p^{l_{1}}-c_{1}, c_{2} p^{l_{1}-l_{2}}, p^{l_{1}}-c_{2} p^{l_{1}-l_{2}}, c_{3} p^{l_{1}-l_{3}}, p^{l_{1}}-c_{3} p^{l_{1}-l_{3}}, c_{4} p^{l_{1}-l_{4}}, p^{l_{1}}-c_{4} p^{l_{1}-l_{4}} \text {. }
$$

If all the following conditions are satisfied:

$$
l_{1}, l_{2}, l_{3}>1, \quad l_{1}>l_{2}, l_{3}, l_{4}, \quad l_{2}>l_{3}, l_{4}, \quad l_{3}>l_{4}, \quad l_{4}>0,
$$


then there are no indices equal to each other $\bmod \left(p^{l_{1}-1}\right)$ and there is no solution of $(1.40)$. So we have to study the cases in which one of them is violated.

$1): l_{1}=1 \geq l_{2} \geq l_{3} \geq l_{4}$. In this case, since the degree of $U(x)$ is less than $p$, and the degree of $P(x)$ is $p-1$, being $U(x)$ divisible by $P(x)$, we must have $U(x)=m P(x)$, for some constant $m$. There are four possibilities:

1.1) : $l_{1}=l_{2}=l_{3}=l_{4}=1$ then $U(0)=0$ and $\mathrm{P}(0)=1$. Then $m=0$ and $U(x) \equiv 0$; moreover if the sum of two (three) terms representing two (three) of the functions $g_{k}$ vanishes, then the sum of the two (three) functions vanishes. As a consequence there are only the following possibilities:

1.1.1) : $g_{i}=-g_{j}$ and $g_{k}=-g_{l}$ for some distinct $i, j, k, l=1, \cdots 4$. This gives rise to the trivial case $(\mathrm{f})$.

1.1.2) $: g_{l}=0$ for some $l=1, \cdots 4$; this is the trivial case $(\mathrm{d})$.

1.1.3) : $U(x)$ contains only two powers of $x$. If $b_{1}, \cdots, b_{4}$ are the coefficients if one of the powers $x^{c}$, then:

$$
b_{1}+b_{2}+b_{3}+b_{4}=0, \quad \text { and } \quad \frac{1}{b_{1}}+\frac{1}{b_{2}}+\frac{1}{b_{3}}+\frac{1}{b_{4}}=0
$$

namely $b_{1}, \cdots, b_{4}$ are the solutions of the following biquadratic equation:

$$
z^{4}+\left(b_{1} b_{2}+b_{1} b_{3}+b_{1} b_{4}+b_{2} b_{3}+b_{2} b_{4}+b_{3} b_{4}\right) z^{2}+b_{1} b_{2} b_{3} b_{4}=0
$$

As a consequence $b_{i}+b_{j}=0, b_{l}+b_{k}=0, \frac{1}{b_{i}}+\frac{1}{b_{j}}=0$ and $\frac{1}{b_{l}}+\frac{1}{b_{k}}=0$, for some distinct $i, j, k, l=1, \cdots, 4$. Then this case reduces to the trivial case (f).

1.2) $l_{1}=l_{2}=l_{3}=1, l_{4}=0$; then $U(0)=\cos 2 \pi \varphi_{4}$ and then $U(x)=\cos 2 \pi \varphi_{4} P(x)$, where $P(x)$ is a polynomial with $p$ powers of $x$. Since in $U$ we have at most 7 powers and $p$ must be prime, then $p$ can only be equal to $2,3,5,7$.

1.2.1) Case $p=2$. Since $p$ is the largest prime in $d_{1}, \cdots, d_{4}$, we must have $d_{1}=d_{2}=d_{3}=$ $d_{4}=2$ and $\delta_{k}=1$. Then $\nu_{k}=0, c_{k}=1$ and this provides no solution.

1.2.2) Case $p=3$. In this case there are the two following possibilities:

$$
\frac{1}{2} e^{2 \pi i f_{1}}+\frac{1}{2} e^{2 \pi i f_{2}}+\frac{1}{2} e^{2 \pi i f_{3}}=\cos 2 \pi \varphi_{4}=\frac{1}{2} e^{-2 \pi i f_{1}}+\frac{1}{2} e^{-2 \pi i f_{2}}+\frac{1}{2} e^{-2 \pi i f_{3}}
$$

or

$$
\frac{1}{2} e^{-2 \pi i f_{1}}+\frac{1}{2} e^{2 \pi i f_{2}}+\frac{1}{2} e^{2 \pi i f_{3}}=\cos 2 \pi \varphi_{4}=\frac{1}{2} e^{2 \pi i f_{1}}+\frac{1}{2} e^{-2 \pi i f_{2}}+\frac{1}{2} e^{-2 \pi i f_{3}} .
$$

In both the case one can show that there are no solutions. In fact, for example, in the first case one has to solve the following equations:

$$
2 \cos 2 \pi \varphi_{4}=\cos 2 \pi f_{1}+\cos 2 \pi f_{2}+\cos 2 \pi f_{3}, \quad \sin 2 \pi f_{1}+\sin 2 \pi f_{2}+\sin 2 \pi f_{3}=0 .
$$

Using the classification of all the possible rational solution (d.1), (d.2), (d.3) of the case $(d)$, one can show that there are no solutions. 
1.2.3) Case $p=5$. In this case we have:

$$
\frac{1}{2} e^{2 \pi i f_{k}}=\frac{1}{2} e^{-2 \pi i f_{k}}=\frac{1}{2} e^{2 \pi i f_{i}}+\frac{1}{2} e^{ \pm 2 \pi i f_{j}}=\cos 2 \pi \varphi_{4},
$$

for some distinct $i, j, k=1,2,3$. Then $f_{k}$ is 0 or $\frac{1}{2}$ and $\varphi_{4}=\frac{1}{6}$ or $\varphi_{4}=\frac{1}{3}$ respectively. Following the same computations of [Cro] we obtain the two solutions (a) and (b).

1.2.4) Case $p=7$. In this case we have:

$$
\frac{1}{2} e^{2 \pi i f_{1}}=\frac{1}{2} e^{-2 \pi i f_{1}}=\frac{1}{2} e^{2 \pi i f_{2}}=\frac{1}{2} e^{-2 \pi i f_{2}}=\frac{1}{2} e^{2 \pi i f_{3}}=\frac{1}{2} e^{-2 \pi i f_{3}}=\cos 2 \pi \varphi_{4},
$$

which has the following solutions:

$$
f_{1}=f_{2}=f_{3}=0 \quad \text { and } \quad \varphi_{4}=\frac{1}{6} \quad \text { or } \quad f_{1}=f_{2}=f_{3}=\frac{1}{2} \quad \text { and } \quad \varphi_{4}=\frac{1}{3}
$$

This gives the solution (c).

1.3) $l_{1}=l_{2}=1$ and $l_{3}=l_{4}=0$. Then $U(x)=\left(\cos 2 \pi \varphi_{3}+\cos 2 \pi \varphi_{4}\right) P(x)$; again in $U$ we have at most 5 powers and then $p=2,3,5$. The case $p=2$ is treated as in [Cro];

1.3.1) : In the case $p=3$ either

$$
\frac{1}{2} e^{2 \pi i f_{1}}+\frac{1}{2} e^{2 \pi i f_{2}}=\frac{1}{2} e^{-2 \pi i f_{1}}+\frac{1}{2} e^{-2 \pi i f_{2}}=\cos 2 \pi \varphi_{3}+\cos 2 \pi \varphi_{4},
$$

or:

$$
\frac{1}{2} e^{2 \pi i f_{1}}+\frac{1}{2} e^{-2 \pi i f_{2}}=\frac{1}{2} e^{-2 \pi i f_{1}}+\frac{1}{2} e^{2 \pi i f_{2}}=\cos 2 \pi \varphi_{3}+\cos 2 \pi \varphi_{4} .
$$

In the former case, for $f_{1}=f_{2}$, with $\cos 2 \pi f_{2}=\cos 2 \pi \varphi_{3}+\cos 2 \pi \varphi_{4}$ and this gives again the solution (b). The latter case is equivalent.

1.3.2) : In the case $p=5$ one has:

$$
\frac{1}{2} e^{2 \pi i f_{1}}=\frac{1}{2} e^{-2 \pi i f_{1}}=\frac{1}{2} e^{2 \pi i f_{2}}=\frac{1}{2} e^{-2 \pi i f_{2}}=\cos 2 \pi \varphi_{3}+\cos 2 \pi \varphi_{4},
$$

which gives $f_{1}=f_{2}=0$ or $f_{1}=f_{2}=\frac{1}{2}$. We treat the former case (the latter is equivalent); then $\cos 2 \pi \varphi_{3}+\cos 2 \pi \varphi_{4}=\frac{1}{2}$ and we can show that this case reduces to the trivial solutions $(\mathrm{d})$ and $(\mathrm{e})$.

1.4) $l_{1}=1$ and $l_{2}=l_{3}=l_{4}=0$. In this case, as in [Cro], there is no solution, but the trivial one $(d)$.

2) $l_{1} \geq 2, l_{1} \geq l_{2}, l_{3}, l_{4}$. This case can be treated as the analogous one in [Cro]. This concludes the proof of Lemma 1.13 .

QED

We now use the above lemma to classify all the triangles which correspond to good triples. Every quadruple generates twelve triangles. In fact, given a solution $\left(\varphi_{1}, \cdots, \varphi_{4}\right)$ we have six ways to choose the pair $\left(\varphi_{i}, \varphi_{j}\right)$ such that

$$
\cos 2 \pi \varphi_{i}+\cos 2 \pi \varphi_{j}=2 \cos \pi\left(\varphi_{i}+\varphi_{j}\right) \cos \pi\left(\varphi_{i}-\varphi_{j}\right)
$$


Chosen the pair $\left(\varphi_{i}, \varphi_{j}\right)$, we have two ways for choosing $\varphi_{k}$, in order to have the triangle

$$
\left(2 \varphi_{k}, \varphi_{i}+\varphi_{j},\left|\varphi_{i}-\varphi_{j}\right|\right) \text {. }
$$

The remaining $\varphi_{l}$ is, by definition, such that the above triangle is mapped, by the braid (1.38), to:

$$
\left(\left|\varphi_{i}-\varphi_{j}\right|,\left|1-\varphi_{i}-\varphi_{j}\right|,\left|1-2 \varphi_{l}\right|\right) .
$$

Let us analyze all the triangles generated by the solutions of the equation (1.40), and keep the good ones, namely the ones for which the new $r_{k}^{\prime}$, given by (1.39), is rational for every $i, j, k$, cyclic permutation of $1,2,3$.

In order to do this, observe that if there exists a permutation $p$ such that the triple $\left(r_{p(1)}, r_{p(2)}, r_{p(3)}\right)$ gives via (1.41) values of $\varphi_{1}, \varphi_{2}, \varphi_{3}$ such that there is not any rational $\varphi_{4}$ such that $\varphi_{1}, \varphi_{2}, \varphi_{3}, \varphi_{4}$ satisfy $(1.40)$, then $\left(r_{1}, r_{2}, r_{3}\right)$ is not a good triple. In fact, every permutation $p$ is generated by ciclic permutations and the permutation $p_{23}:\left(r_{1}, r_{2}, r_{3}\right) \rightarrow$ $\left(r_{1}, r_{3}, r_{2}\right)$. Cyclic permutations are elements of the braid group, so the statement is obvious for them. For $p_{23}$, the statement is a trivial consequence of the fact that the triples $\left(r_{1}, r_{2}, r_{3}\right)$ and $\left(r_{1}, r_{3}, r_{2}\right)$ give via (1.41) the same values of $\varphi_{1}, \varphi_{2}, \varphi_{3}$.

So we will exclude all the triangles $\left(r_{1}, r_{2}, r_{3}\right)$ for which there exists at least a permutation that gives rise to values of $\left(\varphi_{1}, \varphi_{2}, \varphi_{3}\right)$ for which rational solutions $\varphi_{4}$ of (1.40) do not exist.

Solution (a). Using (1.42), we obtain the triangles

$$
\begin{gathered}
\left(\frac{1}{15}, \frac{1}{30}, \frac{23}{30}\right), \quad\left(\frac{1}{15}, \frac{1}{5}, \frac{8}{15}\right), \quad\left(\frac{1}{15}, \frac{7}{30}, \frac{17}{30}\right), \quad\left(\frac{4}{15}, \frac{7}{30}, \frac{13}{30}\right), \\
\left(\frac{11}{15}, \frac{11}{30}, \frac{13}{30}\right), \quad\left(\frac{11}{15}, \frac{2}{15}, \frac{1}{5}\right), \quad\left(\frac{4}{5}, \frac{2}{15}, \frac{1}{5}\right), \quad\left(\frac{1}{5}, \frac{1}{5}, \frac{7}{15}\right), \\
\left(\frac{1}{3}, \frac{11}{30}, \frac{13}{30}\right), \quad\left(\frac{2}{3}, \frac{1}{30}, \frac{7}{30}\right), \quad\left(\frac{1}{3}, \frac{1}{5}, \frac{3}{5}\right), \quad\left(\frac{1}{3}, \frac{1}{3}, \frac{2}{5}\right) .
\end{gathered}
$$

The last two points

$$
\left(\frac{1}{3}, \frac{1}{5}, \frac{3}{5}\right) \quad\left(\frac{1}{3}, \frac{1}{3}, \frac{2}{5}\right)
$$

belong to the orbit (1.37). The above values suitably permuted, except the (1.43), give rise via (1.41), to the following values of $\left(\varphi_{1}, \varphi_{2}, \varphi_{3}\right)$ (written in the same order as the correspondent generating triangles)

$$
\begin{aligned}
& \left(\frac{1}{60}, \frac{5}{12}, \frac{7}{20}\right), \quad\left(\frac{1}{10}, \frac{3}{10}, \frac{7}{30}\right), \quad\left(\frac{7}{60}, \frac{19}{60}, \frac{1}{4}\right), \quad\left(\frac{7}{60}, \frac{7}{20}, \frac{1}{12}\right), \quad\left(\frac{11}{60}, \frac{7}{12}, \frac{3}{20}\right), \\
& \left(\frac{1}{10}, \frac{13}{30}, \frac{3}{10}\right), \quad\left(\frac{1}{15}, \frac{1}{2}, \frac{3}{10}\right), \quad\left(0, \frac{1}{5}, \frac{7}{30}\right), \quad\left(\frac{1}{20}, \frac{11}{60}, \frac{23}{60}\right), \quad\left(\frac{1}{60}, \frac{9}{20}, \frac{13}{60}\right) .
\end{aligned}
$$


there isn't any rational number $\varphi_{4}$ such that any of the quadruples build with these triples and $\varphi_{4}$ is in the class described by Lemma 1.13.

Solution (b). Using (1.42), the triangles are

$$
\begin{gathered}
\left(\frac{7}{15}, \frac{11}{30}, \frac{23}{30}\right), \quad\left(\frac{7}{15}, \frac{2}{5}, \frac{11}{15}\right), \quad\left(\frac{7}{15}, \frac{1}{30}, \frac{11}{30}\right), \quad\left(\frac{2}{15}, \frac{1}{30}, \frac{19}{30}\right), \\
\left(\frac{2}{15}, \frac{1}{30}, \frac{17}{30}\right), \quad\left(\frac{2}{15}, \frac{1}{15}, \frac{3}{5}\right), \quad\left(\frac{2}{5}, \frac{1}{15}, \frac{2}{5}\right), \quad\left(\frac{2}{5}, \frac{11}{15}, \frac{2}{5}\right), \\
\left(\frac{1}{3}, \frac{1}{30}, \frac{13}{30}\right), \quad\left(\frac{1}{3}, \frac{11}{30}, \frac{23}{30}\right), \quad\left(\frac{2}{5}, \frac{1}{3}, \frac{4}{5}\right), \quad\left(\frac{1}{3}, \frac{1}{3}, \frac{4}{5}\right) .
\end{gathered}
$$

The last two points are equivalent to:

$$
\left(\frac{1}{3}, \frac{1}{5}, \frac{3}{5}\right) \quad\left(\frac{2}{3}, \frac{1}{3}, \frac{1}{5}\right)
$$

of the orbit (1.37). As before one can show that if $\left(r_{1}, r_{2}, r_{3}\right)$ is one of the above values, except the (1.44), then there exists a permutation such that the $r_{k}^{\prime}$ defined by (1.39) is no-more rational. In fact we obtain for example the following values of $\left(\varphi_{1}, \varphi_{2}, \varphi_{3}\right)$, which don't fall in the values obtained in Lemma 1.13:

$$
\begin{aligned}
& \left(\frac{11}{60}, \frac{37}{60}, \frac{3}{20}\right), \quad\left(\frac{1}{5}, \frac{3}{5}, \frac{2}{15}\right), \quad\left(\frac{1}{60}, \frac{5}{12}, \frac{1}{20}\right), \quad\left(\frac{1}{60}, \frac{3}{4}, \frac{23}{60}\right), \quad\left(\frac{1}{60}, \frac{47}{60}, \frac{7}{20}\right), \\
& \left(\frac{1}{30}, \frac{23}{30}, \frac{11}{30}\right), \quad\left(\frac{1}{30}, \frac{2}{5}, 0\right), \quad\left(\frac{11}{30}, \frac{2}{5}, 0\right), \quad\left(\frac{1}{60}, \frac{23}{60}, \frac{1}{20}\right), \quad\left(\frac{11}{60}, \frac{11}{20}, \frac{13}{60}\right) .
\end{aligned}
$$

Solution (c).

$$
\begin{gathered}
\left(\frac{2}{7}, \frac{1}{7}, \frac{5}{7}\right),\left(\frac{2}{7}, \frac{5}{42}, \frac{19}{42}\right),\left(\frac{2}{7}, \frac{11}{42}, \frac{25}{42}\right),\left(\frac{4}{7}, \frac{11}{42}, \frac{25}{42}\right),\left(\frac{4}{7}, \frac{2}{7}, \frac{4}{7}\right),\left(\frac{4}{7}, \frac{1}{42}, \frac{13}{42}\right), \\
\left(\frac{1}{7}, \frac{1}{42}, \frac{29}{42}\right),\left(\frac{1}{7}, \frac{5}{42}, \frac{23}{42}\right),\left(\frac{1}{7}, \frac{1}{7}, \frac{4}{7}\right),\left(\frac{1}{3}, \frac{1}{7}, \frac{3}{7}\right),\left(\frac{1}{3}, \frac{2}{7}, \frac{4}{7}\right),\left(\frac{1}{3}, \frac{1}{7}, \frac{5}{7}\right) .
\end{gathered}
$$

As before one can show that if $\left(r_{1}, r_{2}, r_{3}\right)$ is one of the above values then there exists a permutation such that the $r_{k}^{\prime}$ defined by (1.39) is no more rational. In fact we obtain for example the following values of $\left(\varphi_{1}, \varphi_{2}, \varphi_{3}\right)$, which are not included in the values described by Lemma 1.13:

$$
\begin{aligned}
& \left(\frac{1}{14}, \frac{1}{2}, \frac{3}{14}\right), \quad\left(\frac{5}{84}, \frac{31}{84}, \frac{1}{12}\right), \quad\left(\frac{11}{84}, \frac{37}{84}, \frac{13}{84}\right), \quad\left(\frac{11}{84}, \frac{7}{12}, \frac{1}{84}\right), \\
& \left(\frac{1}{7}, \frac{4}{7}, 0\right), \quad\left(\frac{1}{84}, \frac{37}{84}, \frac{11}{84}\right), \quad\left(\frac{1}{84}, \frac{5}{12}, \frac{23}{84}\right), \quad\left(\frac{5}{84}, \frac{29}{84}, \frac{17}{84}\right),
\end{aligned}
$$




$$
\left(\frac{1}{7}, \frac{2}{7}, 0\right), \quad\left(\frac{1}{14}, \frac{8}{21}, \frac{1}{21}\right), \quad\left(\frac{1}{7}, \frac{19}{42}, \frac{5}{42}\right), \quad\left(\frac{1}{14}, \frac{11}{21}, \frac{4}{21}\right) .
$$

Solution (d.1).

$$
\begin{aligned}
& \left(\frac{2}{3}, \frac{3}{20}, \frac{3}{20}\right), \quad\left(\frac{1}{3}, \frac{1}{20}, \frac{9}{20}\right), \quad\left(\frac{1}{5}, \frac{1}{20}, \frac{1}{20}\right), \quad\left(\frac{1}{5}, \frac{1}{30}, \frac{19}{30}\right), \\
& \left(\frac{1}{5}, \frac{1}{12}, \frac{7}{12}\right), \quad\left(\frac{2}{5}, \frac{1}{12}, \frac{5}{12}\right), \quad\left(\frac{3}{5}, \frac{3}{20}, \frac{7}{20}\right), \quad\left(\frac{3}{5}, \frac{7}{30}, \frac{13}{30}\right), \\
& \left(\frac{1}{2}, \frac{7}{30}, \frac{13}{30}\right), \quad\left(\frac{1}{2}, \frac{1}{30}, \frac{11}{30}\right), \quad\left(\frac{2}{3}, \frac{1}{5}, \frac{2}{5}\right), \quad\left(\frac{1}{2}, \frac{1}{5}, \frac{2}{5}\right) \text {. }
\end{aligned}
$$

The last two points are equivalent to:

$$
\left(\frac{1}{3}, \frac{1}{5}, \frac{3}{5}\right) \quad\left(\frac{1}{2}, \frac{2}{5}, \frac{1}{5}\right)
$$

of the orbit (1.37), which is now complete. We can again exclude all the other values of $\left(r_{1}, r_{2}, r_{3}\right)$, with the same trick as above.

Solution (d.2). In this case we have that any of the triangles generated is equivalent to one of the following:

$$
\begin{aligned}
\left(|1-2 \varphi|, \frac{|1-4 \varphi|}{4}\right. & \left., \frac{1+4 \varphi}{4}\right), \quad\left(\frac{1}{2}, \frac{|1-4 \varphi|}{4}, \frac{|3-4 \varphi|}{4}\right), \quad\left(\frac{1}{2}, \frac{|1-4 \varphi|}{4}, \frac{1+4 \varphi}{4}\right), \\
& \left(2 \varphi, \frac{|1-4 \varphi|}{4}, \frac{|3-4 \varphi|}{4}\right), \quad\left(\frac{1}{2}, \frac{1}{2}, \frac{|1-4 \varphi|}{2}\right),
\end{aligned}
$$

where $\varphi$ is an arbitrary rational number. The last triangle is forbidden because it has two right angles, and the first four ones are all equivalent to a flat triangle, so they are again forbidden because they give rise to a an half-integer value of $\mu$.

Solution (d.3). The generated triangles are the following:

$$
\begin{gathered}
\left(\frac{1}{2}, \frac{1}{3}, 2 \varphi+\frac{1}{3}\right), \quad\left(\left|\frac{2}{3}-2 \varphi\right|, \frac{1}{3}, 2 \varphi+\frac{1}{3}\right), \quad\left(\frac{1}{2}, \frac{2}{3}, \frac{2}{3}+2 \varphi\right), \quad\left(\frac{2}{3}+2 \varphi, \frac{2}{3}, \frac{2}{3}+2 \varphi\right), \\
\left(\frac{2}{3}+2 \varphi, \frac{|1-4 \varphi|}{4}, \frac{1+4 \varphi}{4}\right), \quad\left(\left|\frac{2}{3}-2 \varphi\right|, \frac{|1-4 \varphi|}{4}, \frac{1+4 \varphi}{4}\right), \quad\left(\frac{1}{2}, \frac{1}{3}, 1+2 \varphi\right), \\
\left(2 \varphi, \frac{1}{3}, 1+2 \varphi\right), \quad\left(\left|\frac{2}{3}-2 \varphi\right|, \varphi+\frac{1}{12}, \varphi+\frac{7}{12}\right), \quad\left(2 \varphi, \varphi+\frac{1}{12}, \varphi+\frac{7}{12}\right), \\
\left(\frac{2}{3}+2 \varphi, \varphi+\frac{5}{12}, \varphi+\frac{11}{12}\right), \quad\left(2 \varphi, \varphi+\frac{5}{12}, \varphi+\frac{11}{12}\right) .
\end{gathered}
$$


This case must be studied carefully because we have to classify the allowed values of the rational variable $\varphi$ in order that, applying the transformation (1.38), we obtain always rational values.

Let us analyze the first triangle. It is mapped by (1.38) to a triangle equivalent to the second:

$$
\left(\frac{1}{2}, \frac{1}{3}, 2 \varphi+\frac{1}{3}\right) \mapsto\left(\frac{1}{3}+2 \varphi, \frac{2}{3}, 2 \varphi+\frac{1}{3}\right) \sim\left(\left|\frac{2}{3}-2 \varphi\right|, \frac{1}{3}, 2 \varphi+\frac{1}{3}\right) .
$$

If we apply the braid (1.38), with $r_{i}=\frac{2 \pi}{3}, r_{j}=r_{k}=2 \varphi+\frac{1}{3}$, we have to solve:

$$
\cos \frac{2 \pi}{3}+2 \cos ^{2} \pi\left(2 \varphi+\frac{1}{3}\right)=\cos \pi r_{k}^{\prime}
$$

or, equivalently, for $\varphi$ and for the new $\varphi_{k}$ :

$$
\cos \frac{2 \pi}{3}+\cos 2 \pi\left(2 \varphi+\frac{1}{3}\right)+1+\cos 2 \pi \varphi_{k}=0 .
$$

We classify the values of the allowed $\varphi$ using the Lemma 1.13 in the case (e). We have six possibilities for $\varphi$ :

i) if $\varphi_{k}=\frac{1}{2}$, then $\varphi=\frac{11}{24}$. In this case we obtain, from (1.46), all the points of the orbit (1.34).

ii) if $\varphi_{k}=\frac{1}{4}$, then $\varphi=0$. In this case we obtain, from (1.46), all the points of the orbit $(1.33)$.

iii) if $\varphi_{k}=\frac{1}{2}$, then $\varphi=\frac{1}{4}$. In this case we obtain, from (1.46), the following points:

$$
\left(\frac{1}{2}, \frac{1}{3}, \frac{5}{6}\right), \quad\left(\frac{1}{6}, \frac{2}{3}, \frac{1}{6}\right)
$$

We exclude them because there exists a permutation such that the $r_{k}^{\prime}$ defined by (1.39) is no-more rational.

iv) if $\varphi_{k}$ is free to vary, then $2 \varphi+\frac{1}{3}=\frac{1}{2}$. In this case we obtain, from (1.46), the forbidden point:

$$
\left(\frac{1}{2}, \frac{1}{3}, \frac{1}{2}\right)
$$

so we have to exclude it.

v) if $\varphi_{k}=\frac{1}{5}$, then $\varphi=\frac{1}{30}$, and we obtain, from (1.46), the following two points of the orbit (1.36):

$$
\left(\frac{1}{2}, \frac{1}{3}, \frac{2}{5}\right), \quad\left(\frac{2}{5}, \frac{2}{3}, \frac{2}{5}\right)
$$

vi) if $\varphi_{k}=\frac{2}{5}$, then $\varphi=\frac{13}{30}$, and we obtain the following two points of the orbit (1.35):

$$
\left(\frac{1}{2}, \frac{1}{3}, \frac{1}{5}\right), \quad\left(\frac{1}{5}, \frac{2}{3}, \frac{1}{5}\right)
$$


In the same way we can study all the other triangles and show that we don't obtain any other value but the ones described in Theorem 1.6.

Solution (e.1). The generated triangles are the following:

$$
\left(0,0, \frac{2}{3}\right),\left(0, \frac{1}{12}, \frac{7}{12}\right),\left(0, \frac{1}{2}, \frac{2}{3}\right),\left(\frac{2}{3}, \frac{1}{12}, \frac{7}{12}\right)\left(\frac{2}{3}, \frac{1}{3}, \frac{1}{3}\right),\left(\frac{1}{2}, \frac{1}{3}, \frac{1}{3}\right),\left(\frac{2}{3}, \frac{1}{4}, \frac{1}{4}\right) ;
$$

the first four are forbidden because there exists a permutation such that the $r_{k}^{\prime}$ defined by (1.39) is no-more rational. So we have to exclude them. The fifth and the sixth are points of the orbit (1.33) and the last of (1.34).

Solution (e.2). The generated triangles are the following:

$$
\begin{gathered}
\left(2 \varphi, \frac{1}{2}, \frac{1}{2}\right), \quad\left(|1-2 \varphi|, \frac{1}{2}, \frac{1}{2}\right), \quad\left(\frac{1}{2}, \frac{1}{2}, \frac{|1+4 \varphi|}{2}\right), \quad(1, \varphi, \varphi), \\
\left(1, \frac{|1-2 \varphi|}{2}, \frac{|1-2 \varphi|}{2}\right), \quad\left(0, \frac{1}{2}, \frac{|1-4 \varphi|}{2}\right), \quad(|1-2 \varphi|, \varphi, \varphi),\left(0, \frac{|1-2 \varphi|}{2}, \frac{1+2 \varphi}{2}\right), \\
(0, \varphi, 1-\varphi),\left(2 \varphi, \frac{|1-2 \varphi|}{2}, \frac{|1-2 \varphi|}{2}\right),\left(|1-2 \varphi|, \frac{|1-2 \varphi|}{2}, \frac{1+2 \varphi}{2}\right),(2 \varphi, \varphi, 1-\varphi),
\end{gathered}
$$

They are all forbidden, the first three because they have two right angles, the next three ones, because we can prove that necessarily $\varphi=\frac{1}{2}$, then the first has two right angles, the second gives $\left|\cos \pi r_{k}^{\prime}\right|=3$ and the last gives $\left|\cos \pi r_{k}^{\prime}\right|=2$; all the others because they are equivalent to a flat triangle.

Solution (e.3). The generated triangles are the following:

$$
\begin{gathered}
\left(0, \frac{2}{15}, \frac{8}{15}\right),\left(0, \frac{1}{15}, \frac{11}{15}\right),\left(0, \frac{1}{5}, \frac{3}{5}\right),\left(\frac{2}{3}, \frac{1}{5}, \frac{3}{5}\right),\left(\frac{2}{5}, \frac{1}{15}, \frac{11}{15}\right),\left(\frac{4}{5}, \frac{2}{15}, \frac{8}{15}\right), \\
\left(\frac{4}{5}, \frac{1}{5}, \frac{1}{5}\right),\left(\frac{2}{3}, \frac{1}{5}, \frac{1}{5}\right),\left(\frac{2}{3}, \frac{2}{5}, \frac{2}{5}\right),\left(\frac{2}{5}, \frac{2}{5}, \frac{2}{5}\right),\left(\frac{2}{5}, \frac{1}{3}, \frac{1}{3}\right),\left(\frac{1}{5}, \frac{2}{3}, \frac{1}{3}\right) .
\end{gathered}
$$

We exclude the first six because we can show that there exists a permutation such that the $r_{k}^{\prime}$ defined by (1.39) is no-more rational. The seventh and the eighth give two points of the orbit (1.36), the ninth and tenth two points of the orbit (1.39) and the last two, two points of (1.39).

Solution (f) We have obtained all the points of all the orbits of Theorem 1.6. To show that there are no other points we still have to examine the case (f). In this case all the obtained triangles are equivalent to the following:

$$
\begin{gathered}
\left(\frac{\varphi_{1}}{2}, \frac{\left|2-\varphi_{1}+\varphi_{3}\right|}{4}, \frac{\left|2-\varphi_{1}-\varphi_{3}\right|}{4}\right),\left(\frac{\varphi_{1}}{2}, \frac{1}{2}, \frac{\left|1-\varphi_{1}\right|}{2}\right), \\
\left(\frac{\varphi_{1}}{2}, \frac{\left|4-\varphi_{1}-\varphi_{3}\right|}{4}, \frac{\left|\varphi_{1}-\varphi_{3}\right|}{4}\right) .
\end{gathered}
$$


Applying the transformation (1.38) to the above triangles, we find that we have to solve for $\varphi_{1}, \varphi_{3}$ and for the new $\varphi$ obtained from the (1.39), the following three equations respectively:

$$
\begin{gathered}
\cos \pi \frac{\left|2-\varphi_{3}\right|}{2}+\cos \pi \varphi_{1}+\cos 2 \pi \varphi+1=0 \\
\cos \pi \frac{\left|1+\varphi_{1}-\varphi_{3}\right|}{2}+\cos \pi \frac{\left|1-\varphi_{1}-\varphi_{3}\right|}{2}+\cos 2 \pi \varphi=0 \\
2 \cos \pi \frac{\varphi_{1}+\varphi_{3}}{4}+\cos \pi \frac{\left|3 \varphi_{1}-\varphi_{3}\right|}{4}+\cos 2 \pi \varphi=0 .
\end{gathered}
$$

We again can use Lemma 1.13 to prove that we don't obtain any new point. Let us show this in for the first triangle

$$
\left(\frac{\varphi_{1}}{2}, \frac{\left|2-\varphi_{1}+\varphi_{3}\right|}{4}, \frac{\left|2-\varphi_{1}-\varphi_{3}\right|}{4}\right)
$$

We have to solve the equation

$$
\cos \pi \frac{\left|2-\varphi_{3}\right|}{2}+\cos \pi \varphi_{1}+\cos 2 \pi \varphi+1=0
$$

Using Lemma 1.13, the possible values for $\left(\frac{\left|2-\varphi_{3}\right|}{2}, \varphi_{1}, \varphi\right)$ are the (e.1), (e,2) and (e.3). Consider the case (e.1), then the possible solutions for the pair $\left(\varphi_{1}, \varphi_{3}\right)$, are

$$
\left(\varphi_{1}, \varphi_{3}\right)=\left(\frac{1}{2}, \frac{2}{3}\right), \quad\left(\frac{2}{3}, 1\right), \quad\left(\frac{2}{3}, \frac{2}{3}\right) .
$$

Let us substitute these solutions in (1.48); we obtain the triangles

$$
\left(\frac{1}{4}, \frac{13}{24}, \frac{5}{24}\right), \quad\left(\frac{1}{3}, \frac{7}{12}, \frac{1}{12}\right), \quad\left(\frac{1}{3}, \frac{1}{2}, \frac{1}{6}\right),
$$

which are all flat, and thus forbidden. Let us now consider the case (e.2). In this case we obtain two possibilities: either $\varphi_{3}=0$ and $\varphi_{1}$ is a free parameter, or $\varphi_{1}=1$ and $\varphi_{1}$ is a free parameter. In both the cases the triangle (1.48) is flat, and thus forbidden. Let us now consider the last case (e.3). The possible solutions for the pair $\left(\varphi_{1}, \varphi_{3}\right)$, are

$$
\left(\varphi_{1}, \varphi_{3}\right)=\left(\frac{2}{5}, \frac{2}{3}\right), \quad\left(\frac{2}{3}, \frac{6}{5}\right), \quad\left(\frac{2}{5}, \frac{2}{5}\right), \quad\left(\frac{4}{5}, \frac{6}{5}\right), \quad\left(\frac{4}{5}, \frac{2}{3}\right), \quad\left(\frac{2}{3}, \frac{2}{5}\right)
$$

Substituting these values in (1.48), we obtain all flat angles. We can repeat the same proof for the other two triangles in (1.47). In this way we conclude the proof of the theorem. QED

\subsection{Monodromy data and reflection groups.}

We reformulate here the above parameterization of the monodromy data by classes of equivalence of triples $\left(x_{1}, x_{2}, x_{3}\right)$ in a geometric way. Let us consider a three-dimensional 
space $V$ with a basis $\left(e_{1}, e_{2}, e_{3}\right)$ and with a symmetric bilinear form $(\cdot, \cdot)$ given, in this basis, by the matrix

$$
g:=\left(\begin{array}{ccc}
2 & x_{1} & x_{3} \\
x_{1} & 2 & x_{2} \\
x_{3} & x_{2} & 2
\end{array}\right)
$$

namely

$$
\left(e_{i}, e_{i}\right)=2, \quad \text { for } \quad i=1,2,3, \quad \text { and } \quad\left(e_{1}, e_{2}\right)=x_{1}, \quad\left(e_{2}, e_{3}\right)=x_{2}, \quad\left(e_{1}, e_{3}\right)=x_{3} .
$$

Observe that the bilinear form (1.50) does not degenerate. Indeed,

$$
\operatorname{det} g=8-2\left(x_{1}^{2}+x_{2}^{2}+x_{3}^{2}-x_{1} x_{2} x_{3}\right)=8 \cos ^{2} \pi \mu \neq 0,
$$

due to the non-resonance assumption $2 \mu \notin \mathbb{Z}$. The three planes $p_{1}, p_{2}, p_{3}$ orthogonal to the basic vectors $\left(e_{1}, e_{2}, e_{3}\right)$ possess the following properties:

1) The normal vectors to these planes are non-isotropic (i.e. $\left.\left(e_{i}, e_{i}\right) \neq 0\right)$.

2) None of the planes is orthogonal to the other two.

Conversely, a three-dimensional space $V$ with a non-degenerate symmetric bilinear form $(\cdot, \cdot)$ and with an ordered triple of planes satisfying the above conditions, uniquely determines the matrix $g$ of the form (1.50), and then the monodromy data of a solution of $\operatorname{PVI} \mu$.

We define three reflections $R_{1}, R_{2}, R_{3}$ with respect to the three planes $\left(p_{1}, p_{2}, p_{3}\right)$ :

$$
\begin{aligned}
R_{i}: \begin{array}{l}
V \\
x
\end{array} & \mapsto x-\left(e_{i}, x\right) e_{i} \quad i=1,2,3 .
\end{aligned}
$$

These reflection have the following matrix representation in the basis $\left(e_{1}, e_{2}, e_{3}\right)$ :

$$
R_{1}=\left(\begin{array}{ccc}
-1 & -x_{1} & -x_{3} \\
0 & 1 & 0 \\
0 & 0 & 1
\end{array}\right), \quad R_{2}=\left(\begin{array}{ccc}
1 & 0 & 0 \\
-x_{1} & -1 & -x_{2} \\
0 & 0 & 1
\end{array}\right), \quad R_{3}=\left(\begin{array}{ccc}
1 & 0 & 0 \\
0 & 1 & 0 \\
-x_{3} & -x_{2} & -1
\end{array}\right) .
$$

Let us consider the group $G \subset \mathcal{O}(V,(\cdot, \cdot))$ of the linear transformations of $V$, generated by the three reflections $R_{1}, R_{2}, R_{3}$. The matrix $g$ will be called the Gram matrix of the reflection group $G$. It determines the subgroup $G \subset \mathcal{O}(V,(\cdot, \cdot))$ uniquely. We observe that, for an admissible triple, the group $G$ is irreducible, namely there are no non-trivial subspaces of $V$ which are invariant with respect to all the transformations of $G$.

We conclude that the branches of the solutions of $\mathrm{PVI} \mu$ can be parameterized by groups $G \subset \mathcal{O}(3)$ with a marked ordered system of generating reflections $R_{1}, R_{2}, R_{3}$. Let us describe what happens with the triples of generators under the analytic continuation of the solution.

We define an action of the braid group $B_{3}$ on the systems of generators $R_{1}, R_{2}, R_{3}$ of the reflection group $G$ :

$$
\begin{aligned}
& \beta_{1}:\left(R_{1}, R_{2}, R_{3}\right) \mapsto\left(R_{1}, R_{2}, R_{3}\right)^{\beta_{1}}:=\left(R_{2}, R_{2} R_{1} R_{2}, R_{3}\right), \\
& \beta_{2}:\left(R_{1}, R_{2}, R_{3}\right) \mapsto\left(R_{1}, R_{2}, R_{3}\right)^{\beta_{2}}:=\left(R_{1}, R_{3}, R_{3} R_{2} R_{3}\right),
\end{aligned}
$$

where $\beta_{1,2}$ are the standard generators of the braid group. Observe that the groups generated by the reflections $\left(R_{1}, R_{2}, R_{3}\right)$ and $\left(R_{1}, R_{2}, R_{3}\right)^{\beta}$ coincide for any $\beta \in B_{3}$. In particular the following lemma holds true: 
Lemma 1.15. For any braid $\beta \in B_{3}$, the transformations $\beta\left(R_{1}, R_{2}, R_{3}\right)$ are reflections with respect to some planes orthogonal to some new basic vectors $\left(e_{1}^{\beta}, e_{2}^{\beta}, e_{3}^{\beta}\right)$. The Gram matrix with respect to the basis $\left(e_{1}^{\beta}, e_{2}^{\beta}, e_{3}^{\beta}\right)$ has the form:

$$
\left(e_{i}^{\beta}, e_{i}^{\beta}\right)=2, \quad i=1,2,3, \quad\left(e_{1}^{\beta}, e_{2}^{\beta}\right)=x_{1}^{\beta}, \quad\left(e_{2}^{\beta}, e_{3}^{\beta}\right)=x_{2}^{\beta}, \quad\left(e_{1}^{\beta}, e_{3}^{\beta}\right)=x_{3}^{\beta},
$$

where $\left(x_{1}^{\beta}, x_{2}^{\beta}, x_{3}^{\beta}\right)=\beta\left(x_{1}, x_{2}, x_{3}\right)$.

Proof. It is sufficient to check the statement for the generators $\beta_{1,2}$. For $\beta=\beta_{1}$ :

$$
e_{1}^{\beta_{1}}=e_{2}, \quad e_{2}^{\beta_{1}}=e_{1}-x_{1} e_{2}, \quad e_{3}^{\beta_{1}}=e_{3}
$$

for $\beta=\beta_{2}$ :

$$
e_{1}^{\beta_{2}}=e_{1}, \quad e_{2}^{\beta_{2}}=e_{3}, \quad e_{3}^{\beta_{2}}=e_{2}-x_{2} e_{3}
$$

Computing the Gram matrix we prove the lemma.

1.4.1. Reflection groups and algebraic solutions Let us figure out what are the reflection groups corresponding to the finite orbits classified in Theorem 1.6.

Theorem 1.7. The orbit (1.33) corresponds to the group $W\left(A_{3}\right)$ of symmetries of regular tetrahedron, the orbit (1.34) corresponds to the group $W\left(B_{3}\right)$ of symmetries of the regular octahedron, the orbits (1.35), (1.36), (1.37) correspond to different choices of a system of generating reflections in the group $W\left(\mathrm{H}_{3}\right)$ of symmetries of icosahedron.

Proof. It is sufficient to find one point in each of the orbits $(1.33),(1.34),(1.35),(1.36)$, (1.37) that corresponds to a triple of symmetry planes of a regular polyhedron. To this end, we associate to a regular polyhedron a standard triple of symmetry planes using the following construction. Let 0 be the center of the polyhedron. Take a face of the polyhedron and denote $H$ the center of this face, $P$ a vertex and $Q$ the center of an edge of the face passing through the vertex $P$. The standard triple consists of the symmetry planes trough the points $O P Q, O Q H, O H P$ respectively. Let us compute the angles between the planes of each regular polyhedron. It is convinient to use the Schläfli symbol $\{p, q\}$ for regular polyhedra (see $[\mathrm{Cox}]$ ). In these notations, the face of the regular polyhedron $\{p, q\}$ is a regular $p$-gon, the vertex figure is a regular $q$-gon. We immediately see that the angles between the planes of the standard triple are

$\begin{array}{cccc}\text { between } O P Q \text { and } O Q H & \frac{\pi}{2}, \\ \text { between } O Q H \text { and } O H P & \frac{\pi}{p}, \\ \text { between } O H P \text { and } O P Q & \frac{\pi}{q} .\end{array}$

So, for the tetrahedron $\{3,3\}$ we obtain the angles $\left(\frac{\pi}{2}, \frac{\pi}{3}, \frac{\pi}{3}\right)$, for the octahedron $\{3,4\}$ the angles $\left(\frac{\pi}{2}, \frac{\pi}{3}, \frac{\pi}{4}\right)$, for the icosahedron $\{3,5\}$ the angles $\left(\frac{\pi}{2}, \frac{\pi}{3}, \frac{\pi}{5}\right)$. In this way, we obtain the triples $(1.33),(1.34),(1.35)$. The reciprocal polyherda (i.e. cube $\{4,3\}$ and dodecaherdon 
$\{5,3\})$ give the same angles up to permutations. As we already know, the permuted triples of (1.34) or (1.35) belong to the same orbit. So, the standard triples of cube and dodecahedron are $B_{3}$-equivalent to those of octahedron and icosahedron respectively.

To obtain the last two orbits (1.36) and (1.37), we apply the above construction of the standard triple to great icosahedron and dodecahedron respectively. These non convex regular polyhedra both have icosaherdal symmetry (see [Cox]). Their Schläfli symbols are $\left\{3, \frac{5}{2}\right\}$ and $\left\{5, \frac{5}{2}\right\}$ respectively. This means that the faces of these polyhedra are regular triangles or pentagons, but the vertex figures are pentagrams. The above computation gives the triples (1.36) and (1.37). Again we need not consider the reciprocal stellated polyhedra. Theorem 1.7 is proved.

QED

1.4.2. Classification of the monodromy data, second proof. We present here another proof of Theorem 1.6, based on the idea suggested by E. B. Vinberg. We start with the following:

Algebraic Lemma. Let $(x, y, z)$ be an admissible triple of real numbers, satisfying the inequalities:

$$
x^{2}+y^{2}+z^{2}-x y z>4,
$$

and

$$
|x|,|y|,|z| \leq 2 .
$$

Then there exists a braid $\beta \in B_{3}$ such that the absolute value of some of the coordinates of $\beta(x, y, z)$ is strictly greater than 2 .

Before proving the lemma, we observe that we can assume, without loss of generality, that all the coordinates of $(x, y, z)$ are non-zero; in fact, for any admissible triple, there exists a braid $\beta \in B_{3}$ such that all the coordinates of $\beta(x, y, z)$ are non-zero. Let us denote $b_{x}, b_{y}$ and $b_{z}$ the following braids:

$$
\begin{array}{ccc}
b_{x}:= & \beta_{2}, & b_{x}(x, y, z)=(z,-x, x-y z), \\
b_{y}:= & \beta_{2}^{-1} \beta_{1} \beta_{2}, & b_{y}(x, y, z)=(-y+x z,-x,-z), \\
b_{z}:= & \beta_{1}, & b_{z}(x, y, z)=(-x, z-x y, y) .
\end{array}
$$

Lemma 1.16. Let $(x, y, z)$ be a triple of non-zero real numbers, satisfying

$$
0<|z|,|x|,|y| \leq 2
$$

and

$$
x^{2}+y^{2}+z^{2}-x y z=4+c^{2}, \quad c>0 .
$$

Denote $\left(x^{\prime}, y^{\prime}, z^{\prime}\right):=\beta(x, y, z)$, where

$$
\beta=\left\{\begin{array}{lll}
b_{x} & \text { if } & |x| \leq|y|,|z|, \\
b_{y} & \text { if } & |y| \leq|x|,|z|, \\
b_{z} & \text { if } & |z| \leq|x|,|y| .
\end{array}\right.
$$


Then:

$$
\min \left\{\left|x^{\prime}\right|,\left|y^{\prime}\right|,\left|z^{\prime}\right|\right\} \geq \min \{|x|,|y|,|z|\}
$$

and

$$
\left|x^{\prime}\right|+\left|y^{\prime}\right|+\left|z^{\prime}\right| \geq|x|+|y|+|z|+\min \left\{z^{2}, 2 c\right\} .
$$

Proof. Let us prove the lemma in the case where $|z| \leq|x|,|y|$ and $\beta=b_{z}$. The other cases can be proved in the same way. If the signs of $z$ and of $x y$ are opposite then

$$
\left|y^{\prime}\right|=|z|+|x y| \geq|z|+z^{2}, \quad\left|x^{\prime}\right|=|x|, \quad\left|z^{\prime}\right|=|z|
$$

and (1.57), (1.58) are proved. Let us suppose that the signs of $z$ and of $x y$ are the same. Changing the triple $(x, y, z)$ to an equivalent one, we can assume that all the coordinates are positive. If we prove now that

$$
2 z+2 c \leq x y,
$$

where $c$ is given in (1.56), we have that $\left|y^{\prime}\right|=|x y-z| \geq z+2 c$ and the lemma is proved. To prove (1.59) we find the constrained minimum of the function $x y$ on the domain $D$ defined by the conditions (1.55) and (1.56). The Lagrange function

$$
F(x, y, z):=x y-\lambda\left(x^{2}+y^{2}+z^{2}-x y z\right),
$$

has the local maximum at

$$
x=y=\sqrt{\frac{4+c^{2}-z^{2}}{2-z}},
$$

and no minimum in the interior of $D$. It remains to study the values of the function $x y$ on the boundary of $D$. If, say, $z=y$ then the positive root $x$ of the equation

$$
x^{2}+2 z^{2}-x z^{2}=4+c^{2}
$$

is greater than 2. So the boundaries $z=y$ and $z=x$ are not reached for $(x, y, z) \in D$, and then $|z|<|x|,|y|$. It remains the last boundary to be studied. If, say, $y=2$, we find $x=z \pm c$. Since $x \geq z$, then $x=z+c$ and $x y=2(z+c)$; this is the minimum of the function $x y$.

QED

Proof of Algebraic Lemma. As observed above we can always reduce to the case where all the coordinates $(x, y, z)$ are non-zero. Put:

$$
\Delta(x, y, z):=\min \left\{x^{2}, y^{2}, z^{2}, 2 \sqrt{x^{2}+y^{2}+z^{2}-x y z-4}\right\} .
$$

Using Lemma 1.16, we can build a braid $b_{1}$ such that the coordinates:

$$
\left(x_{1}, y_{1}, z_{1}\right):=b_{1}(x, y, z)
$$

satisfy the inequalities

$$
\min \left\{\left|x_{1}\right|,\left|y_{1}\right|,\left|z_{1}\right|\right\} \geq \min \{|x|,|y|,|z|\} \quad\left|x_{1}\right|+\left|y_{1}\right|+\left|z_{1}\right| \geq|x|+|y|+|z|+\Delta(x, y, z) .
$$


Since the quantity $x^{2}+y^{2}+z^{2}-x y z-4$ is preserved by the action of the braid group, we obtain:

$$
\Delta\left(x_{1}, y_{1}, z_{1}\right) \geq \Delta(x, y, z) .
$$

If the absolute value of some of the coordinates $\left(x_{1}, y_{1}, z_{1}\right)$ is greater than 2 , the lemma in proved. Otherwise we apply again the construction of Lemma 1.16 to the triple $\left(x_{1}, y_{1}, z_{1}\right)$. In this way we obtain a sequence of braids $b_{1}, b_{2}, b_{3} \cdots$ such that the corresponding triples

$$
\left(x_{k+1}, y_{k+1}, z_{k+1}\right):=b_{k+1}\left(x_{k}, y_{k}, z_{k}\right)
$$

satisfy

$$
\Delta\left(x_{k+1}, y_{k+1}, z_{k+1}\right) \geq \Delta\left(x_{k}, y_{k}, z_{k}\right) .
$$

Iterating the inequality (1.57), we obtain that

$$
\left|x_{k}\right|+\left|y_{k}\right|+\left|z_{k}\right| \geq|x|+|y|+|z|+k \Delta(x, y, z) .
$$

Hence, in a finite number of steps we build a triple such that the absolute value of at least one of the coordinates in greater than 2. This concludes the proof of Algebraic Lemma. QED

Corollary 1.4. For an algebraic solution of PVI $\mu$, specified by an admissible triple $x_{i}=$ $-2 \cos 2 \pi r_{i}$, the value of $\mu$ must be real, the strict inequalities

$$
\left|x_{i}\right|<2, \quad i=1,2,3
$$

hold true and the matrix $g$ defined in (1.50) is positive definite.

Proof. Let us prove that, for an algebraic solution, the triple $\left(x_{1}, x_{2}, x_{3}\right)$ must satisfy the inequality:

$$
x_{1}^{2}+x_{2}^{2}+x_{3}^{2}-x_{1} x_{2} x_{3}<4 .
$$

Indeed, if $x_{1}^{2}+x_{2}^{2}+x_{3}^{2}-x_{1} x_{2} x_{3}>4$, then, according to the Algebraic Lemma the triple is not a good one. This contradicts the assumption that the solution is algebraic. If $x_{1}^{2}+x_{2}^{2}+x_{3}^{2}-x_{1} x_{2} x_{3}=4$, then $\mu=\frac{1}{2}+k$ with $k \in \mathbb{Z}$. This contradicts the basic assumption $2 \mu \notin \mathbb{Z}$. Then (1.62) is satisfied and $\mu$ is a real number. Let us now prove (1.61). If one of the coordinates, say $x_{1}$, is such that $x_{1}= \pm 2$, then

$$
x_{1}^{2}+x_{2}^{2}+x_{3}^{2}-x_{1} x_{2} x_{3}=4+\left(x_{2} \mp x_{3}\right)^{2},
$$

and, being $x_{2} x_{3}$ real numbers, (1.62) is violated. So, $x_{i} \neq \pm 2$ for every $i$. Finally, applying the Sylvester criterion to the matrix $g$, we prove that $g$ is positive definite. In fact

$$
\operatorname{det} G=8-2\left(x_{1}^{2}+x_{2}^{2}+x_{3}^{2}-x_{1} x_{2} x_{3}\right)>0,
$$

and for any principal minor

$$
\operatorname{det}\left(\begin{array}{cc}
2 & x_{i} \\
x_{i} & 2
\end{array}\right)=4-x_{i}^{2}>0 .
$$

QED 
Lemma 1.17. For an algebraic solution of $P V I \mu$ the reflection group $G$ acts in the Euclidean space.

The proof immediately follows from the fact that the correspondent Gram matrix is positive definite.

Corollary 1.5. For a good triple $\left(x_{1}, x_{2}, x_{3}\right)$ and for any braid $\beta \in B_{3}$, there exists three integer positive numbers $n_{12}^{\beta}, n_{13}^{\beta}$ and $n_{23}^{\beta}$ such that:

$$
\left(R_{i}^{\beta} R_{j}^{\beta}\right)^{n_{i j}^{\beta}}=\mathbf{1}, \quad \text { for } \quad i \neq j, \quad i, j=1,2,3
$$

Proof. If $\left(e_{1}, e_{2}\right)=x_{1}=-2 \cos \pi r$ with $r=\frac{m}{n}, m, n \in \mathbb{Z}$, then $R_{1} R_{2}$ is a rotation by the angle $2 \pi \frac{m}{n}$. Hence:

$$
\left(R_{1} R_{2}\right)^{n}=\mathbf{1}
$$

This holds true for any pair $R_{i}$ and $R_{j}$. Moreover, for any braid $\beta \in B_{3}$, the triple $\beta\left(x_{1}, x_{2}, x_{3}\right)$ is again good, then (1.63) is proved.

QED

Corollary 1.6. The set of the solutions of the PVI $\mu$ equation with a real non resonant value of $\mu$ and real parameters $\left(x_{1}, x_{2}, x_{3}\right)$ satisfying

$$
\left|x_{i}\right|<2, \quad i=1,2,3
$$

is invariant with respect to the analytic continuation.

Proof. Applying the Sylvester criterion to the matrix $g$ defined in (1.50), we obtain that $g$ is positive definite. So the reflections $R_{1}, R_{2}, R_{3}$ can be realized in the Euclidean space. After a transformation $\left(x_{1}, x_{2}, x_{3}\right) \mapsto\left(x_{1}^{\beta}, x_{2}^{\beta}, x_{3}^{\beta}\right)=\beta\left(x_{1}, x_{2}, x_{3}\right)$, the new numbers $\left(x_{1}{ }^{\beta}, x_{2}{ }^{\beta}, x_{3}{ }^{\beta}\right)$ are the entries of the Gram matrix:

$$
g^{\beta}:=\left(\begin{array}{ccc}
2 & x_{1}^{\beta} & x_{3}^{\beta} \\
x_{1}^{\beta} & 2 & x_{2}^{\beta} \\
x_{3}^{\beta} & x_{2}^{\beta} & 2
\end{array}\right)
$$

of the basis $\left(e_{1}^{\beta}, e_{2}^{\beta}, e_{3}^{\beta}\right)$, in the same Euclidean space. Then this matrix must be positive definite, namely $x_{i}^{2}<4$ as we wanted to prove.

QED

In the second part of this paper, we will identify the set described in Corollary 1.6 with the class of solutions of PVI $\mu$ having asymptotic behaviour of algebraic type. This identification will be crucial in the computation of the five algebraic solutions of PVI $\mu$ we have classified.

As we have just shown, a good triple

$$
\left(x_{1}, x_{2}, x_{3}\right)=\left(-2 \cos \pi \frac{m_{1}}{n_{1}},-2 \cos \pi \frac{m_{2}}{n_{2}},-2 \cos \pi \frac{m_{3}}{n_{3}}\right),
$$


corresponds to a representation of the Coxeter group generated by three reflections $R_{1}$, $R_{2}, R_{3}$ satisfying

$$
R_{1}^{2}=R_{2}^{2}=R_{3}^{2}=\mathbf{1}, \quad\left(R_{1} R_{2}\right)^{n_{1}}=\left(R_{2} R_{3}\right)^{n_{2}}=\left(R_{1} R_{3}\right)^{n_{3}}=\mathbf{1},
$$

in the three-dimensional Euclidean space. We denoted by $G$ the image of this representation. Moreover, for any braid $\beta \in B_{3}$, the matrices

$$
\left(R_{1}^{\beta}, R_{2}^{\beta}, R_{3}^{\beta}\right):=\beta\left(R_{1}, R_{2}, R_{3}\right),
$$

satisfy the same identities (1.64), with some new integers $n_{1}^{\beta}, n_{2}^{\beta}, n_{3}^{\beta}$. We stress that the reflections $R_{1}^{\beta}, R_{2}^{\beta}, R_{3}^{\beta}$ generate the same group $G$.

Theorem 1.8. It follows from the above property that $G$ is an irreducible finite Coxeter group.

Let $n$ be the least common multiple of $n_{1}, n_{2}$ and $n_{3}$. Put:

$$
\zeta=2 \cos \frac{\pi}{n}
$$

Lemma 1.18. The numbers

$$
x_{i}=-2 \cos \pi \frac{m_{i}}{n_{i}}, \quad i=1,2,3
$$

belong to the ring $\mathcal{K}_{0}$ of integers of the field $\mathcal{K}:=\mathbf{Q}[\zeta]$.

Recall (see $\left[\right.$ Wey]) that $\mathcal{K}$ is the normal extension of $\mathbf{Q}$ generated by $\zeta$ and $\mathcal{K}_{0}$ is the ring of all the algebraic integer numbers of $\mathcal{K}$, namely it consists of all the elements $x \in \mathcal{K}$ satisfying an algebraic equation of the form

$$
x^{k}+a_{1} x^{k-1}+\cdots+a_{k}=0, \quad \text { with } \quad a_{i} \in \mathbb{Z} .
$$

Proof of Lemma 1.18. Let $n=n_{i} m_{i}^{\prime}$, then

$$
\cos \pi \frac{m_{i}}{n_{i}}=T_{m_{i} m_{i}^{\prime}}\left(\cos \frac{\pi}{n}\right)=T_{m_{i} m_{i}^{\prime}}\left(\frac{1}{2} \zeta\right),
$$

where

$$
T_{k}(x)=\cos (k \arccos x)=2^{k-1} x^{k}+\sum_{s=0}^{k-1} 2^{s-1} a_{k s} x^{s},
$$

are the Tchebyscheff polynomials of the first kind (see [Bat]). Recall that all the coefficients $a_{k s}$ are integers, so $x_{i}=-2 \cos \pi \frac{m_{i}}{n_{i}}$ is a polynomial of $\zeta$ with integer coefficients. Moreover $\zeta$ is a root of the monic algebraic equation with integer coefficients

$$
2 T_{n}\left(\frac{\zeta}{2}\right)+2=\zeta^{n}+\sum_{s=0}^{n-1} a_{n s} \zeta^{s}+2=0
$$


Hence $\zeta \in \mathcal{K}_{0}$ and $x_{i}=-2 T_{m_{i} m_{i}^{\prime}}\left(\frac{\zeta}{2}\right) \in \mathcal{K}_{0}$, as we wanted to prove.

Proof of Theorem 1.8. From the formulae (1.51) it follows that the matrices $R_{1}, R_{2}$ and $R_{3}$ are all defined over the same ring $\mathcal{K}_{0}$ of integers of $\mathcal{K}$ :

$$
R_{i} \in \operatorname{Mat}\left(\mathcal{K}_{0}, 3\right) .
$$

Moreover, these matrices are orthogonal with respect to $g$ :

$$
R_{i}^{T} g R_{i}=g
$$

where $g$ is defined in (1.50). Let

$$
\Gamma:=\operatorname{Gal}(\mathcal{K}, \mathbf{Q})
$$

the Galois group of $\mathcal{K}$ over $\mathbf{Q}$, namely the group of all automorphisms

$$
\phi: \mathcal{K} \rightarrow \mathcal{K}
$$

identical on $\mathbf{Q}$.

For any $\phi \in \Gamma$ we denote $\phi\left(R_{i}\right)$ and $\phi(g)$ the matrices obtained from $R_{i}$ and $g$ by the action

$$
\left(x_{1}, x_{2}, x_{3}\right) \mapsto\left(\phi\left(x_{1}\right), \phi\left(x_{2}\right), \phi\left(x_{3}\right)\right) .
$$

Lemma 1.19. For any $\phi \in \Gamma$ the following statements hold true:

i) $\operatorname{det} \phi(g) \neq 0$,

ii) The matrices $\phi\left(R_{i}\right)$ are orthogonal with respect to $\phi(g)$.

iii) For any $\beta \in B_{3}$ the matrices $\phi\left(R_{i}\right)^{\beta}$ satisfy the Coxeter relation (1.63).

The proof is obvious, due to the fact that any automorphism preserves all the algebraic relations.

From the above lemma, and from Algebraic Lemma, it follows that for any $\phi \in \Gamma$, the real symmetric matrix $\phi(g)$ must be positive definite. We will show that this implies that the group $G$ is finite. Let $N$ be the order of the Galois group $\Gamma$. We construct the block-diagonal matrices

$$
\mathcal{R}_{i} \in \operatorname{Mat}\left(\mathcal{K}_{0}, 3 N\right), \quad i=1,2,3,
$$

as the matrices formed by $3 \times 3$ blocks on the diagonal, such that the $j$-th block is $\phi_{j}\left(R_{i}\right)$, for $\phi_{j} \in \Gamma, j=1,2, \cdots, N$. The matrices $\mathcal{R}_{i}$ are orthogonal with respect to $\mathcal{G}$, that is the block-diagonal matrix having $\phi_{j}(G)$, for $\phi_{j} \in \Gamma, j=1,2, \cdots, N$, on the diagonal blocks. We can apply Lemma 1.19 to the matrices $\mathcal{R}_{i}$ to show that they satisfy the Coxeter relation (1.63). As a consequence we obtain a representation of our reflection group $G$ into the orthogonal group

$$
\begin{aligned}
G & \rightarrow \mathcal{O}\left(\mathcal{K}^{3 N}, \mathcal{G}\right) \\
R_{i} & \mapsto \mathcal{R}_{i} .
\end{aligned}
$$

By construction the matrices $\mathcal{R}_{i}$ preserve the sublattice

$$
\mathcal{K}_{0}^{3 N} \subset \mathcal{K}^{3 N}
$$


of the vectors the components of which are algebraic integers of the field $\mathcal{K}$. We recall (see [Wey]) that the ring $\mathcal{K}_{0}$ of the algebraic integers of the field $\mathcal{K}$, is a finite-dimensional lattice. As a consequence, the image of the representation (1.68) is a discrete subgroup of the orthogonal group. Since $\mathcal{G}$ is positive definite, the orthogonal group is compact and, hence, $G$ must be finite. The theorem is proved.

QED

To complete the classification of the monodromy data related to the algebraic solutions it remains to classify the objects

$$
\left(G, R_{1}, R_{2}, R_{3}\right)
$$

where $G$ is one of the Coxeter groups $A_{3}, B_{3}$ and $H_{3}$ and $\left(R_{1}, R_{2}, R_{3}\right)$ is a triple of generating reflections considered modulo the action (1.52) of the braid group. This can be done by a straightforward computation of all the orbits of the triples of generating reflections. All of them were described and classified by Schwartz (see the introduction). We arrive again at the list of Theorem 1.6, where, as we already know, the triples (1.33) generate the group $W\left(A_{3}\right)$ of the symmetries of the tetrahedron, (1.34) generate the group $W\left(B_{3}\right)$ of the symmetries of the cube, while $(1.35),(1.36)$ and (1.37) correspond to three inequivalent triples of the generating reflections of the group $W\left(H_{3}\right)$.

\section{GLOBAL STRUCTURE OF THE SOLUTIONS OF PAINLEVE' VI $\mu$ HAV- ING CRITICAL BEHAVIOUR OF ALGEBRAIC TYPE}

In the first part of this paper, we found a class of solutions of PVI $\mu$ invariant with respect to the analytic continuation. For them, the reflection group $G$ acts in the three-dimensional Euclidean space. Recall that the parameter $\mu$ must be real, the coordinates of the admissible triples $\left(x_{1}, x_{2}, x_{3}\right)$ must be real and satisfy the inequality

$$
-2<x_{i}<2, \quad i=1,2,3 \text {. }
$$

In this second part, we prove that this class of solutions coincides with the class of the solutions of PVI $\mu$ having critical behaviour of the algebraic type



where $\varepsilon>0$ is small enough, the indices $l_{0}, l_{1}, l_{\infty}$ are real and the coefficients $a_{0}, a_{1}, a_{\infty}$ are some complex numbers. We compute the behaviour of any branch of these solutions near the critical points. These results will be used to compute explicitly all the algebraic solutions classified in the first part. 
First of all, we fix the notations. Let us choose:

$$
u_{1}=0, \quad u_{2}=x, \quad u_{3}=1 .
$$

Then the Fuchsian system (1.1) reads

$$
\frac{\mathrm{d}}{\mathrm{d} z} Y=\mathcal{A}(z, x) Y=\left(\frac{\mathcal{A}_{1}}{z}+\frac{\mathcal{A}_{2}}{z-x}+\frac{\mathcal{A}_{3}}{z-1}\right) Y,
$$

and, putting

$$
\mathcal{A}_{1}:=A_{0}, \quad \mathcal{A}_{2}:=A_{x}, \quad \mathcal{A}_{3}:=A_{1}, \quad \mathcal{A}_{\infty}=A_{\infty},
$$

we obtain

$$
\frac{\mathrm{d}}{\mathrm{d} z} Y=\left(\frac{A_{0}}{z}+\frac{A_{1}}{z-1}+\frac{A_{x}}{z-x}\right) Y .
$$

The branch cuts in $\overline{\mathbb{C}}$ are the same as in Section 1.1. We call now the basic loops $\gamma_{0}, \gamma_{x}, \gamma_{1}$. They are fixed as before, namely $\gamma_{0}, \gamma_{x}, \gamma_{1}$ play the role of the preceding $\gamma_{1}, \gamma_{2}, \gamma_{3}$ (see figure 1). The Schlesinger equations read:

$$
\begin{aligned}
\frac{\mathrm{d}}{\mathrm{d} x} A_{0}(x) & =-\frac{\left[A_{0}, A_{x}\right]}{x}, \\
\frac{\mathrm{d}}{\mathrm{d} x} A_{1}(x) & =-\frac{\left[A_{1}, A_{x}\right]}{x-1}, \\
\frac{\mathrm{d}}{\mathrm{d} x} A_{x}(x) & =\frac{\left[A_{0}, A_{x}\right]}{x}+\frac{\left[A_{1}, A_{x}\right]}{x-1} .
\end{aligned}
$$

The correspondent monodromy matrices are

$$
M_{0}, \quad M_{x}, \quad M_{1},
$$

which play the role of the preceding $M_{1}, M_{2}, M_{3}$ respectively. We recall that they satisfy

$$
M_{\infty} M_{1} M_{x} M_{0}=\mathbf{1}, \quad \operatorname{det}\left(M_{i}\right)=1, \quad \operatorname{Tr}\left(M_{i}\right)=2, \quad \text { for } \quad i=0,1, x,
$$

with

$$
M_{\infty}=\left(\begin{array}{cc}
\exp (2 i \pi \mu) & 0 \\
0 & \exp (-2 i \pi \mu)
\end{array}\right) .
$$

With the above choice of $A_{\infty}, A_{1}, A_{x}$ and $A_{0}$, satisfying

$$
\operatorname{det} A_{i}=0, \quad \operatorname{Tr}\left(A_{i}\right)=0, \quad i=0,1, \infty,
$$

the non-singular solution $A(z, x)$ of the Schlesinger equations turns out to be related to the solution of PVI $\mu$ in the following way (see [JMU]):

$$
[A(y, x)]_{12}=0, \quad \text { iff } \quad y(x) \text { solves } \operatorname{PVI} \mu,
$$

where $y$ is not identically equal to $0,1, x$.

We now state the first main theorem of this second part: 
Theorem 2.1. For any admissible triple $\left(x_{0}, x_{1}, x_{\infty}\right), x_{i} \in \mathbb{R},\left|x_{i}\right|<2$ for $i=0,1, \infty$, there exists a unique branch $y\left(x ; x_{0}, x_{1}, x_{\infty}\right)$ of a solution of $P V I \mu$, with the parameter $\mu$ satisfying the equation:

$$
4 \sin ^{2} \pi \mu=x_{0}^{2}+x_{1}^{2}+x_{\infty}^{2}-x_{0} x_{1} x_{\infty},
$$

with the asymptotic behaviour (2.1) near the critical points $0,1, \infty$. The indices are given by

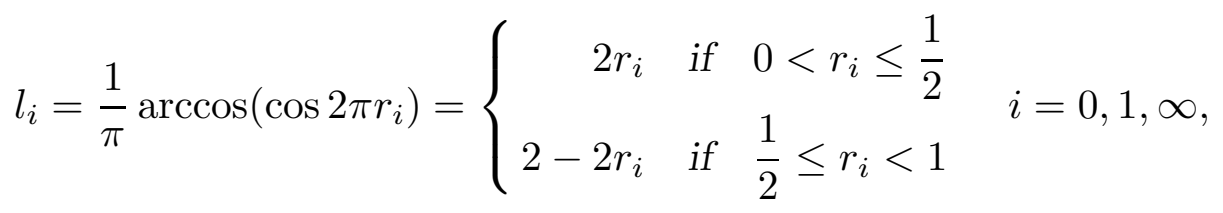

with

$$
x_{i}=-2 \cos \pi r_{i}, \quad i=0,1, \infty,
$$

and the leading coefficients $a_{0}, a_{1}, a_{\infty}$ are single-valued functions of the equivalence class of $x_{0}, x_{1}, x_{\infty}$ and of $\mu$. Namely, the coefficient $a_{0}$, for $x_{0} \neq 0$, is given by:

$$
a_{0}=\frac{\exp (-i \pi \phi)}{4\left(2 \mu+l_{0}-1\right)^{2}} \frac{\Gamma^{2}\left(1-l_{0}\right) \Gamma^{2}\left(\frac{1+l_{0}}{2}\right) \Gamma\left(\frac{1+l_{0}}{2}+\mu\right) \Gamma\left(\frac{1+l_{0}}{2}-\mu\right)}{\Gamma^{2}\left(l_{0}\right) \Gamma^{2}\left(\frac{1-l_{0}}{2}\right) \Gamma\left(\frac{1-l_{0}}{2}+\mu\right) \Gamma\left(\frac{1-l_{0}}{2}-\mu\right)}
$$

where

$$
\exp (i \pi \phi)=\frac{x_{0}^{2} x_{1}^{2}-2 x_{1}^{2}-2 x_{0} x_{1} x_{\infty}+2 x_{\infty}^{2}+i x_{1} \operatorname{sign}\left(x_{0}\right) \sqrt{4-x_{0}^{2}}\left(2 x_{\infty}-x_{0} x_{1}\right)}{2\left(x_{1}^{2}-x_{0} x_{1} x_{\infty}+x_{\infty}^{2}\right)}
$$

and for $x_{0}=0$

$$
a_{0}=\frac{x_{\infty}^{2}}{x_{1}^{2}+x_{\infty}^{2}}
$$

The coefficient $a_{1}$ is given by the same formula with the substitution $x_{0} \leftrightarrow x_{1}, l_{0} \mapsto l_{1} ; a_{\infty}$ is given by the same formula too, after the substitution $\left(x_{0}, x_{1}, x_{\infty}\right) \mapsto\left(x_{\infty},-x_{1}, x_{0}-x_{1} x_{\infty}\right)$ and $l_{0} \mapsto l_{\infty}$. Conversely any solution of the PVI $\mu$ equation, with a real value of $\mu$, having critical behaviour of algebraic type, can be obtained by the above construction.

Remark 2.1. The relation (2.8) determines $\mu$ up to the transformations

$$
\mu \mapsto \pm \mu+n, \quad n \in \mathbb{Z} .
$$

According to the results of Section 1.2, such an ambiguity can be absorbed by the action of a symmetry on PVI $\mu$. Recall that these symmetries preserve the class of the algebraic solutions.

Theorem 2.1 will be proved in Section 2.4.

\subsection{Local theory of the solutions of $\operatorname{PVI} \mu$ having critical behaviour of algebraic type}

2.1.1. Local asymptotic behaviour around 0 . In this section we characterize the local asymptotic behaviour of the solutions of PVI $\mu$ near the singular point $x=0$. First of all let us characterize the type of asymptotic behaviour that can be related to the algebraic solutions. 
Lemma 2.1. Let $y(x)$ be an algebraic solution of PVI $\mu$. Then the first term of its Puiseux series is

$$
y(x) \sim a_{0} x^{1-\sigma_{0}} \quad \text { as } \quad x \rightarrow 0 .
$$

for some constant $a_{0} \neq 0$ and the rational number $\sigma_{0}$ must satisfy $0 \leq \sigma_{0}<1$, with $a_{0} \neq 1$ if $\sigma_{0}=0$.

Proof. If $y(x)$ is an algebraic function, then it admits an expansion in Puiseux series around 0

$$
y(x)=\sum_{k=k_{0}}^{\infty} a_{k} x^{\frac{k}{n}}, \quad k_{0} \in \mathbb{Z}, \quad a_{k_{0}} \neq 0,
$$

where $n$ is some natural number. As a consequence, for $k_{0} \neq 0$, we have the following relation between the orders of the first and second derivative of $y$ :

$$
\mathcal{O}\left(x^{2} y^{\prime \prime}\right)=\mathcal{O}\left(x y^{\prime}\right)=\mathcal{O}(y)=\mathcal{O}\left(x^{\frac{k_{0}}{n}}\right)
$$

We now reduce to the common denominator the PVI $\mu$ equation and collect together all the terms of the same order in the numerator $\mathcal{N}$, using the rule (2.14). The numerator is

$$
\begin{aligned}
\mathcal{N}= & 2 y^{\prime 2} x^{4}-y^{\prime 2} x^{3}-y^{\prime 2} x^{5}+2 y^{\prime 2} x^{2} y-2 y^{\prime} x^{3} y-2 y^{\prime 2} x^{3} y+2 y^{\prime \prime} x^{3} y+2 y^{\prime} x^{4} y-2 y^{\prime 2} x^{4} y- \\
& -4 y^{\prime \prime} x^{4} y+2 y^{\prime 2} x^{5} y+2 y^{\prime \prime} x^{5} y+x y^{2}-2 y^{\prime} x y^{2}-2 x^{2} y^{2}+4 \mu x^{2} y^{2}-4 \mu^{2} x^{2} y^{2}+ \\
& +6 y^{\prime} x^{2} y^{2}-3 y^{\prime 2} x^{2} y^{2}-2 y^{\prime \prime} x^{2} y^{2}-2 y^{\prime} x^{3} y^{2}+6 y^{\prime 2} x^{3} y^{2}+2 y^{\prime \prime} x^{3} y^{2}-2 y^{\prime} x^{4} y^{2}- \\
& -3 y^{\prime 2} x^{4} y^{2}+2 y^{\prime \prime} x^{4} y^{2}-2 y^{\prime \prime} x^{5} y^{2}-8 \mu x y^{3}+8 \mu^{2} x y^{3}+2 y^{\prime} x y^{3}+4 x^{2} y^{3}- \\
& -8 \mu x^{2} y^{3}+8 \mu^{2} x^{2} y^{3}-6 y^{\prime} x^{2} y^{3}+2 y^{\prime \prime} x^{2} y^{3}+4 y^{\prime} x^{3} y^{3}-4 y^{\prime \prime} x^{3} y^{3}+2 y^{\prime \prime} x^{4} y^{3}- \\
& -y^{4}+4 \mu y^{4}-4 \mu^{2} y^{4}-3 x y^{4}+16 \mu x y^{4}-16 \mu^{2} x y^{4}-2 x^{2} y^{4}+4 \mu x^{2} y^{4}- \\
& -4 \mu^{2} x^{2} y^{4}+2 y^{5}-8 \mu y^{5}+8 \mu^{2} y^{5}+2 x y^{5}-8 \mu x y^{5}+8 \mu^{2} x y^{5}-y^{6}+4 \mu y^{6}-4 \mu^{2} y^{6} .
\end{aligned}
$$

The first term of the Puiseux series must be chosen in order to kill the lowest term in the numerator of the PVI $\mu$ equation. If $k_{0}<0$, the lowest term is

$$
-y^{6}+4 \mu y^{6}-4 \mu^{2} y^{6}
$$

which, for $2 \mu \notin \mathbb{Z}$ cannot be zero for any choice of $a_{0} \neq 0$. Then $k_{0}$ cannot be negative. If $n \geq k_{0}>0$, the lowest order term is

$$
2 x^{2} y^{\prime 2} y-2 x y^{\prime} y^{2}-2 x^{2} y^{\prime \prime} y^{2}
$$

which is zero for any $y=a_{k_{0}} x^{\frac{k_{0}}{n}}$. For $k_{0}>n$, the lowest order term is

$$
-x^{3} y^{\prime 2}+2 x^{3} y^{\prime \prime} y+x y^{2}
$$

which cannot be zero. Furthermore, for $k_{0}=0$, the lowest order term in the numerator $\mathcal{N}$ is

$$
-a_{0}^{4}\left(a_{0}-1\right)^{2}(2 \mu-1)^{2}
$$


and, due to the assumptions $2 \mu \notin \mathbb{Z}$ and $a_{0} \neq 0$, the only possible value of $a_{0}$ is 1 . Substituting $y=1+a_{1} x^{\frac{k_{1}}{n}}$, we obtain that the lowest order term in the numerator $\mathcal{N}$ is

$$
x^{\frac{2 k_{1}}{n}} a_{1}^{2}\left(k_{1} / n+1-2 \mu\right)\left(k_{1} / n-1+2 \mu\right)
$$

that is zero, for generic values of $\mu$, only if $a_{1}$ is 0 . If $\mu=\frac{1}{2} \pm \frac{k_{1}}{2 n}$, we can again repeat the procedure. The numerator will be

$$
\begin{aligned}
\hat{\mathcal{N}}= & -4 \hat{\mu}^{2} \hat{y}^{2}-16 \hat{\mu}^{2} \hat{y}^{3}-24 \hat{\mu}^{2} \hat{y}^{4}-16 \hat{\mu}^{2} \hat{y}^{5}-4 \hat{\mu}^{2} \hat{y}^{6}+\hat{y}^{2} x+8 \hat{\mu}^{2} \hat{y}^{2} x+2 \hat{y}^{3} x+24 \hat{\mu}^{2} \hat{y}^{3} x+ \\
& +\hat{y}^{4} x+24 \hat{\mu}^{2} \hat{y}^{4} x+8 \hat{\mu}^{2} \hat{y}^{5} x+2 \hat{y} y^{\prime} x+4 \hat{y}^{2} y^{\prime} x+2 \hat{y}^{3} y^{\prime} x-\hat{y}^{2} x^{2}-4 \hat{\mu}^{2} \hat{y}^{2} x^{2}-2 \hat{y}^{3} x^{2}- \\
& -8 \hat{\mu}^{2} \hat{y}^{3} x^{2}-\hat{y}^{4} x^{2}-4 \hat{\mu}^{2} \hat{y}^{4} x^{2}-6 \hat{y} y^{\prime} x^{2}-12 \hat{y}^{2} y^{\prime} x^{2}-6 \hat{y}^{3} y^{\prime} x^{2}-y^{2} x^{2}-4 \hat{y} y^{2} x^{2}- \\
& -3 \hat{y}^{2} y^{\prime 2} x^{2}+2 \hat{y} y^{\prime \prime} x^{2}+4 \hat{y}^{2} y^{\prime \prime} x^{2}+2 \hat{y}^{3} y^{\prime \prime} x^{2}+6 \hat{y} y^{\prime} x^{3}+10 \hat{y}^{2} y^{\prime} x^{3}+4 \hat{y}^{3} y^{\prime} x^{3}+ \\
& +3 y^{\prime 2} x^{3}+10 \hat{y} y^{\prime 2} x^{3}+6 \hat{y}^{2} y^{\prime 2} x^{3}-6 \hat{y} y^{\prime \prime} x^{3}-10 \hat{y}^{2} y^{\prime \prime} x^{3}-4 \hat{y}^{3} y^{\prime \prime} x^{3}-2 \hat{y} y^{\prime} x^{4}- \\
& -2 \hat{y}^{2} y^{\prime} x^{4}-3 y^{\prime 2} x^{4}-3 \hat{y}^{2} y^{\prime 2} x^{4}+6 \hat{y} y^{\prime \prime} x^{4}+8 \hat{y}^{2} y^{\prime \prime} x^{4}+2 \hat{y}^{3} y^{\prime \prime} x^{4}+y^{2} x^{5}+ \\
& +2 \hat{y} y^{\prime 2} x^{5}-2 \hat{y} y^{\prime \prime} x^{5}-2 \hat{y}^{2} y^{\prime \prime} x^{5}
\end{aligned}
$$

where $\hat{\mu}= \pm \frac{k_{1}}{2 n}$ and $\hat{y}=y-1$. Substituting $\hat{y}=a_{1} x^{\frac{k_{1}}{n}}$, the lowest order term in the numerator $\hat{\mathcal{N}}$ is automatically zero. Now, we want to eliminate the next lowest order term. Observe thet, now

$$
\mathcal{O}\left(x^{2} y^{\prime \prime}\right)=\mathcal{O}\left(x y^{\prime}\right)=\mathcal{O}(\hat{y})=\mathcal{O}\left(x^{\frac{k_{1}}{n}}\right)
$$

For the sake of definitess, suppose $\frac{1}{2}<\mu \leq 1$, i.e. $\hat{\mu}=\frac{k_{1}}{2 n}<\frac{1}{2}$ (the case $\hat{\mu}=-\frac{k_{1}}{2 n}$ is analogous). The next lowest order terms in the numerator $\hat{\mathcal{N}}$ are

$$
-16 \hat{\mu}^{2} \hat{y}^{3}+4 x \hat{y}^{2} y^{\prime}-4 x^{2} \hat{y} y^{\prime 2}+4 x^{2} \hat{y}^{2} y^{\prime \prime}+x \hat{y}^{2}+8 x \hat{\mu}^{2} \hat{y}^{2}-6 x^{2} \hat{y} y^{\prime}+3 x^{3} y^{\prime 2}-6 x^{3} \hat{y} y^{\prime \prime}
$$

To eliminate them, we substitute $y=1+a_{1} x^{\frac{k_{1}}{n}}+a_{1} y^{\frac{k_{2}}{n}}$, for some $k_{2}>k_{1}$. The above terms give

$$
-4 a_{1}^{3}\left(\frac{k_{1}}{n}\right)^{2} x^{3 \frac{k_{1}}{n}}+\mathcal{O}\left(x^{1+2 \frac{k_{1}}{n}}\right)
$$

that is zero if and only of $a_{1}=0$. So we obtain the forbidden solution $y(x) \equiv 1$. So, $k_{0}$ can not be zero, and $y(x)$ satisfies (2.13) with $0<l=\frac{k_{0}}{n} \leq 1$, namely $0 \leq \sigma_{0}<1$. QED

In the above lemma we have seen the expected asymptotic behaviour of the algebraic solutions. We now state the main result of this section, which is more general, namely it holds also for non algebraic solutions.

Theorem 2.2. For any pair of values $\left(a_{0}, \sigma_{0}\right), 0 \leq \sigma_{0}<1$, there exists a unique branch of the solution of PVI $\mu$, for a fixed $\mu$, with the asymptotic behaviour

$$
y(x)=a_{0} x^{1-\sigma_{0}}\left(1+x^{\varepsilon} f(x)\right) \quad \text { as } \quad x \rightarrow 0,
$$


for some $\varepsilon>0$ and $f(x)$ smooth function such that $\lim _{x \rightarrow 0} f(x)=$ const.

In order that $x^{1-\sigma_{0}}$ is well defined, we have to make some cut in the complex plane. From now on, we cut along the line $\arg x=\varphi$ for some $\varphi$.

Remark 2.2. Theorem 2.2 can be proved also for complex values of the index $\sigma_{0}$, provided that $0 \leq \operatorname{Re} \sigma_{0}<1$. For algebraic solutions the index $\sigma_{0}$ must be a rational number. Because of this, we consider only real indices.

2.1.2. Proof of the existence. First of all we state the existence of solutions of the Schlesinger equations with a particular asymptotic behaviour. The following result will play an important role also in Section 2.3.

Lemma 2.2 (Sato-Miwa-Jimbo). Given three constant matrices $A_{i}^{0}, i=0,1, x$ with zero eigenvalues such that $\Lambda=A_{0}^{0}+A_{x}^{0}$ has eigenvalues $\pm \frac{\sigma}{2}, 0 \leq \sigma<1$, and $A_{1}^{0}=-\Lambda-A_{\infty}$, in any sector of $\overline{\mathbb{C}}$ containing none of the branch cuts, and sufficiently close to 0 , there exists a solution of the Schlesinger equations that satisfy

$$
\begin{aligned}
\left|A_{1}(x)-A_{1}^{0}\right| \leq K|x|^{1-\sigma^{\prime}} & \left|x^{-\Lambda}\left(A_{1}(x)-A_{1}^{0}\right) x^{\Lambda}\right| \leq K|x|^{1-\sigma^{\prime}} \\
\left|x^{-\Lambda} A_{0}(x) x^{\Lambda}-A_{0}^{0}\right| \leq K|x|^{1-\sigma^{\prime}} & \left|x^{-\Lambda} A_{x}(x) x^{\Lambda}-A_{x}^{0}\right| \leq K|x|^{1-\sigma^{\prime}}
\end{aligned}
$$

where $K$ is some positive constant and $1>\sigma^{\prime}>\sigma$.

We want to show that it is possible to choose $A_{0,1, x}$ and $\Lambda$ such that the corresponding solution $y(x)$ of the Painlevé VI equation obtained via (2.7) has the asymptotic behaviour (2.15). Let us consider an arbitrary constant matrix $\Lambda$ with eigenvalues $\pm \frac{\sigma}{2}$; let $T$ be the diagonalizing matrix of $\Lambda$, namely

$$
\Lambda=T\left(\begin{array}{cc}
\frac{\sigma}{2} & 0 \\
0 & -\frac{\sigma}{2}
\end{array}\right) T^{-1}
$$

Now, we choose $A_{1}^{0}=-A_{\infty}-\Lambda$ and $A_{0, x}^{0}$ such that $A_{0}^{0}+A_{x}^{0}=\Lambda$, namely

$$
A_{0}^{0}=\frac{1}{2} \Lambda+F, \quad A_{x}^{0}=\frac{1}{2} \Lambda-F
$$

for some constant matrix $F$. Then:

$$
T^{-1} A_{0}^{0} T=\left(\begin{array}{cc}
\frac{\sigma}{4} & 0 \\
0 & -\frac{\sigma}{4}
\end{array}\right)+E, \quad T^{-1} A_{x}^{0} T=\left(\begin{array}{cc}
\frac{\sigma}{4} & 0 \\
0 & -\frac{\sigma}{4}
\end{array}\right)-E,
$$

where we can choose $E=\left(\begin{array}{cc}0 & \frac{b \sigma}{4} \\ \frac{-\sigma}{4 b} & 0\end{array}\right)$, for some non-zero constant $b$. With this choice of $E, A_{0}$ and $A_{x}$ have zero eigenvalues. Using Lemma 2.2, we obtain that, as $x \rightarrow 0$ :

$$
A_{0, x} \rightarrow T\left(\begin{array}{cc}
x^{\frac{\sigma}{2}} & 0 \\
0 & x^{\frac{-\sigma}{2}}
\end{array}\right)\left[\left(\begin{array}{cc}
\frac{\sigma}{4} & 0 \\
0 & -\frac{\sigma}{4}
\end{array}\right) \pm\left(\begin{array}{cc}
0 & \frac{b \sigma x^{\sigma}}{4} \\
\frac{-\sigma x^{-\sigma}}{4 b} & 0
\end{array}\right)\right]\left(\begin{array}{cc}
x^{\frac{-\sigma}{2}} & 0 \\
0 & x^{\frac{\sigma}{2}}
\end{array}\right) T^{-1},
$$


and

$$
A_{1} \rightarrow-A_{\infty}-\Lambda \text {. }
$$

Substituting such asymptotic behaviors in the relation (2.7), taking $T_{12}, T_{11} \neq 0$ we obtain:

$$
y(x) \sim-\frac{T_{12} x^{1-\sigma}}{4 b T_{11}}
$$

we are now free to choose the arbitrary constants $b, T_{11}, T_{12}, \sigma$ in such a way that $-\frac{T_{12}}{4 b T_{11}}=$ $a_{0}, \sigma=\sigma_{0}$, for any fixed $a_{0}$ and $\sigma_{0}$.

Remark 2.3. Other existence results for $\sigma \in \mathbb{C} \backslash\{]-\infty, 0] \cup[1,+\infty[\}$ can be found in [IKSY] and [S1], [S2], [S3]. For indices with $\operatorname{Re} \sigma \notin[0,1]$, the asymptotics obtained in these papers are valid in more complicated domains near 0.

2.1.3. Proof of the uniqueness. Now we prove that the solution $y(x), x \in \mathcal{B}(0, r)$, of Painlevé VI equation such that it satisfies (2.15) for some given constants $a_{0}$ and $\sigma_{0} \in[0,1)$, is uniquely determined by $a_{0}$ and $\sigma_{0}$. Here $\mathcal{B}(0, r)=\{x|| x \mid \leq r, \arg x \neq \varphi, x \neq 0\}$.

The proof is based on the fact that Painlevé VI is equivalent to the following reduced Schlesinger equations (1.17):

$$
\begin{gathered}
\dot{q}=\frac{(q-1) q+2 p(q-1) q(q-x)}{(x-1) x} \\
\dot{p}=\frac{-p^{2}\left(x-2 q-2 x q+3 q^{2}\right)-p(2 q-1)-(1-\mu) \mu}{(x-1) x}
\end{gathered}
$$

where:

$$
q=y, \quad p=\frac{x(x-1) \dot{y}-y(y-1)}{2(y-x) y(y-1)},
$$

and the dot means the derivative $\frac{\mathrm{d}}{\mathrm{d} x}$ We shall prove the local uniqueness of the solutions of the Hamiltonian system with the following asymptotic behaviour

$$
q(x) \sim a x^{l}+x^{l+\varepsilon} f(x) \quad p(x) \sim \frac{l-1}{2 a} \frac{1}{x^{l}}+\frac{x^{\varepsilon} g(x)}{x^{l}}
$$

where $l=1-\sigma_{0}, a=a_{0}, \varepsilon>0$ and $f(x)$ and $g(x)$ are some smooth functions in $\mathcal{B}(0, r)$ which tend to zero as $x \rightarrow 0$.

This is equivalent to show the theorem. In fact, from the uniqueness of $q$ it follows trivially the uniqueness of $y$. The following lemma holds true:

Lemma 2.3. The estimates (2.20) on the asymptotic behaviour of $(q(x), p(x))$ are a consequence of (2.15).

Proof. Since $q=y$, the assertion on $y$ is obvious due to the hypothesis (2.15). Concerning $p$, we use its definition

$$
p=\frac{x(x-1) \dot{y}-y(y-1)}{2(y-x) y(y-1)}
$$


and by a straightforward computation we show (2.20) for p.

We now distinguish two cases: $0<l<1$, and $l=1$. Let us consider the former case; it is convenient to introduce the new variables $(\tilde{q}, \tilde{p})$

$$
\tilde{q}=\frac{y}{x^{l}} \quad \tilde{p}=x^{l} p
$$

which have a similar asymptotic behaviour

$$
\tilde{q}(x)=a+x^{\varepsilon} f(x) \quad \tilde{p}(x)=\frac{l-1}{2 a}+x^{\varepsilon} g(x),
$$

and the equations of the motion become

$$
\begin{aligned}
& \dot{\tilde{q}}=f_{q}\left(\tilde{p}, \tilde{q}, x, x^{l}\right), \\
& \dot{\tilde{p}}=f_{p}\left(\tilde{p}, \tilde{q}, x, x^{l}\right),
\end{aligned}
$$

with

$$
f_{q}=\frac{-\tilde{q}(l-1-2 \tilde{p} \tilde{q})}{x}-\frac{\tilde{q}(1+2 \tilde{p} \tilde{q})}{x^{1-l}}+\frac{\tilde{q}(1+4 \tilde{p} \tilde{q})-2 x^{-l} \tilde{p} \tilde{q}-x^{l} \tilde{q}^{2}(1+2 \tilde{p} \tilde{q})}{1-x}
$$

and

$f_{p}=\frac{\tilde{p}(l-1-2 \tilde{p} \tilde{q})}{x}+\frac{\mu-\mu^{2}+2 \tilde{p} \tilde{q}+3 \tilde{p}^{2} \tilde{q}^{2}}{x^{1-l}}+\frac{\frac{\tilde{p}^{2}}{x^{l}}-\tilde{p}(1+4 \tilde{p} \tilde{q})+x^{l}\left(\mu-\mu^{2}+2 \tilde{p} \tilde{q}+3 \tilde{p}^{2} \tilde{q}^{2}\right)}{1-x}$

We want to prove the uniqueness of the solution $(\tilde{q}, \tilde{p})$ of $(2.22)$, satisfying (2.21) for $x \in \mathcal{B}(0, r)$, in the ball $\left\|\tilde{p}-\frac{l-1}{2 a}\right\|,\|\tilde{q}-a\| \leq C_{r}$, for a constant $C_{r}$ vanishing when the radius $r \rightarrow 0$. Here $\|f\|=\sup _{\mathcal{B}(0, r)}|f(x)|$. Let us suppose that there are two solutions $\left(\tilde{q}_{1}, \tilde{p}_{1}\right)$ and $\left(\tilde{q}_{2}, \tilde{p}_{2}\right)$ of the system $(2.22)$, satisfying $(2.21)$. Then, if we define $\mathrm{X}=\left(\begin{array}{c}\tilde{q}_{1}-\tilde{q}_{2} \\ \tilde{p}_{1}-\tilde{p}_{2}\end{array}\right)$, we obtain, as a consequence of (2.21), that the following limits exist

$$
\lim _{|x| \rightarrow 0, \arg (x)=\vartheta} \frac{\mathrm{X}^{(i)}(x)}{|x|^{\varepsilon}}=0, \quad i=1,2,
$$

for some $0<\varepsilon, \mathrm{X}^{(i)}$ being the $\mathrm{i}$-th component of $\mathrm{X}$. Moreover, $\mathrm{X}$ satisfies the following

$$
\mathrm{X}^{\prime}=\left(\begin{array}{c}
\frac{\left[1-l+2 \tilde{p}_{1}\left(\tilde{q}_{1}+\tilde{q}_{2}\right)\right]\left(\tilde{q}_{1}-\tilde{q}_{2}\right)+2 \tilde{q}_{2}^{2}\left(\tilde{p}_{1}-\tilde{p}_{2}\right)}{x}+\frac{\Delta \mathrm{Q}_{1}}{x^{1-l}}+\frac{\Delta \mathrm{Q}_{2}}{x^{l}}+\Delta \mathrm{Q}_{3} \\
\frac{\left[l-1-2 \tilde{q}_{2}\left(\tilde{p}_{1}+\tilde{p}_{2}\right)\right]\left(\tilde{p}_{1}-\tilde{p}_{2}\right)-2 \tilde{p}_{1}^{2}\left(\tilde{q}_{1}-\tilde{q}_{2}\right)}{x}+\frac{\Delta \mathrm{P}_{1}}{x^{1-l}}+\frac{\Delta \mathrm{P}_{2}}{x^{l}}+\Delta \mathrm{P}_{3}
\end{array}\right),
$$

where

$$
\Delta \mathrm{Q}_{i}=Q_{i}\left(\tilde{q}_{1}, \tilde{p}_{1}, x\right)-Q_{i}\left(\tilde{q}_{2}, \tilde{p}_{2}, x\right), \quad \text { and } \quad \Delta \mathrm{P}_{i}=P_{i}\left(\tilde{q}_{1}, \tilde{p}_{1}, x\right)-P_{i}\left(\tilde{q}_{2}, \tilde{p}_{2}, x\right),
$$


$Q_{1}=\tilde{q}(1+2 \tilde{p} \tilde{q}), Q_{2}=-2 \tilde{p} \tilde{q}, Q_{3}=\frac{\tilde{q}(1+4 \tilde{q} \tilde{q})-2 x^{1-l} \tilde{p} \tilde{q}-x^{l} \tilde{q}^{2}(1+2 \tilde{p} \tilde{q})}{1-x}, P_{1}=\mu-\mu^{2}+2 \tilde{p} \tilde{q}+3 \tilde{p}^{2} \tilde{q}^{2}$ $P_{2}=\tilde{p}^{2}, P_{3}=\frac{p^{2} x^{1-l}-\tilde{p}(1+4 \tilde{p} \tilde{q})+x^{l}\left(\mu-\mu^{2}+2 \tilde{p} \tilde{q}+3 \tilde{p}^{2} \tilde{q}^{2}\right)}{1-x}$.

We want to prove that, under the hypothesis (2.23), $\mathrm{X} \equiv 0$ (this is equivalent to prove our theorem). Performing the constant linear transformation $\mathrm{X}=\mathrm{T} Z$, where

$$
\mathrm{T}=\left(\begin{array}{cc}
1 & 0 \\
\frac{1-l}{2 a^{2}} & \frac{1}{2 a^{2}}
\end{array}\right)
$$

we obtain

$$
\mathrm{Z}^{\prime}=\left(\begin{array}{c}
\underline{\left[1-l+2 \tilde{p}_{1}\left(\tilde{q}_{1}+\tilde{q}_{2}\right)\right]\left(\tilde{q}_{1}-\tilde{q}_{2}\right)+2 \tilde{q}_{2}^{2}\left(\tilde{p}_{1}-\tilde{p}_{2}\right)}+\frac{\Delta \mathrm{Q}_{1}}{x}+\frac{\Delta \mathrm{Q}_{2}}{x^{l}}+\Delta \mathrm{Q}_{3} \\
\frac{2 a^{2} G\left(\tilde{p}_{1}, \tilde{p}_{2}, \tilde{q}_{1}, \tilde{q}_{2}\right)}{x}+\frac{2 a^{2} \Delta \mathrm{P}_{1}+(l-1) \Delta \mathrm{Q}_{1}}{x^{1-l}}+\frac{2 a^{2} \Delta \mathrm{P}_{2}+(l-1) \Delta \mathrm{Q}_{2}}{x^{l}}+2 a^{2} \Delta \mathrm{P}_{3}+(l-1) \Delta \mathrm{Q}_{3}
\end{array}\right)
$$

where

$$
\begin{aligned}
G\left(\tilde{p}_{1}, \tilde{p}_{2}, \tilde{q}_{1}, \tilde{q}_{2}\right)= & {\left.\left[l-1-2 \tilde{q}_{2}\left(\tilde{p}_{1}+\tilde{p}_{2}\right)\right]\left(\tilde{p}_{1}-\tilde{p}_{2}\right)-2 \tilde{p}_{1}^{2}\left(\tilde{q}_{1}-\tilde{q}_{2}\right)\right\}+ } \\
& +(l-1)\left\{\left[1-l+2 \tilde{p}_{1}\left(\tilde{q}_{1}+\tilde{q}_{2}\right)\right]\left(\tilde{q}_{1}-\tilde{q}_{2}\right)+2 \tilde{q}_{2}^{2}\left(\tilde{p}_{1}-\tilde{p}_{2}\right),\right.
\end{aligned}
$$

and, from (2.23):

$$
\lim _{|x| \rightarrow 0, \arg (x)=\vartheta} \frac{Z^{(i)}(x)}{|x|^{\varepsilon}}=0, \quad i=1,2 .
$$

In order to prove that $\mathrm{Z} \equiv 0$, we fix any direction in the complex plane $\arg (x)=\vartheta$ for some fixed $\vartheta$, and we consider the real variable $t=|x|$. Then we define:

$$
\mathrm{V}^{(i)}(t):=\left|Z^{(i)}(x)\right| \text {. }
$$

We want to prove that the assumption $\mathrm{V}^{(i)}\left(t_{0}\right) \neq 0$ for some $t_{0}>0$ leads to a cotradiction. To this aim we prove a differential inequality for the right derivative $D_{+} \mathrm{V}^{(i)}$ of $V^{(i)}(t)$. Since $D_{+} \mathrm{V}^{(i)} \leq\left|\mathrm{Z}^{(i)^{\prime}}\right|$, to obtain such a differential inequality it is enough to estimate from above the modulus of the components of the right-hand-side of (2.24). To this aim we notice that all the polynomials $Q_{i}, P_{i}$ have the form:

$$
Q_{i}=\sum_{k, n=0}^{3} a_{k, n}^{i} \tilde{p}^{n} \tilde{q}^{k}, \quad P_{i}=\sum_{k, n=0}^{3} b_{k, n}^{i} \tilde{p}^{n} \tilde{q}^{k},
$$

with $a_{k, n}(x), b_{k, n}(x)$ regular functions $x \in \mathcal{B}(0, r)$. As a consequence, we obtain, in the ball $\left\|\tilde{p}-\frac{l-1}{2 a}\right\|,\|\tilde{q}-a\| \leq C_{r}$, the estimates:

$$
\left|\Delta \mathrm{Q}_{i}\right|, \quad\left|\Delta \mathrm{P}_{i}\right| \leq c_{1}^{i}\left|\mathrm{Z}^{(1)}\right|+c_{2}^{i}\left|\mathrm{Z}^{(2)}\right|
$$

for some positive constants $c_{1}^{i}, c_{2}^{i}$. In fact

$$
\begin{aligned}
&\left|\Delta \mathrm{Q}_{i}\right|=\left|\sum_{k, n} a_{k, n}\left[\tilde{q}_{1}^{k}\left(\tilde{p}_{1}^{n}-\tilde{p}_{2}^{n}\right)+\tilde{p}_{2}^{n}\left(\tilde{q}_{1}^{k}-\tilde{q}_{2}^{k}\right)\right]\right| \leq\left\{\sum_{k=0,1,2} C_{r}^{(1)^{k}}\left(\| a_{k, 1}||+2 C_{r}^{(2)}|| a_{k, 2}||\right)\right\} \\
& \cdot\left|\tilde{p}_{1}-\tilde{p}_{2}\right|+\left\{\sum_{n=0,1,2,3} C_{r}^{(2)^{n}}\left(\left\|a_{1, n}||+2 C_{r}^{(1)}|| a_{2, n}\right\|+3 C_{r}^{(1)^{2}}|| a_{3, n} \|\right)\right\} \cdot\left|\tilde{q}_{1}-\tilde{q}_{2}\right|,
\end{aligned}
$$


where $C_{r}^{(1)}=C_{r}+2|a|$ and $C_{r}^{(2)}=C_{r}+\frac{1-l}{|a|}$. We obtain (2.26) observing that $\left|\tilde{q}_{1}-\tilde{q}_{2}\right|$, $\left|\tilde{p}_{1}-\tilde{p}_{2}\right|$ are related to $\left|\mathrm{Z}^{(1)}\right|,\left|\mathrm{Z}^{(2)}\right|$ by the constant linear transformation $T$.

For the terms of order $\mathcal{O}\left(\frac{1}{x}\right)$ in $(2.24)$ we have:

$$
\begin{aligned}
\frac{\left|\left[1-l+2 \tilde{p}_{1}\left(\tilde{q}_{1}+\tilde{q}_{2}\right)\right]\left(\tilde{q}_{1}-\tilde{q}_{2}\right)+2 \tilde{q}_{2}^{2}\left(\tilde{p}_{1}-\tilde{p}_{2}\right)\right|}{|x|} \leq & \frac{\left|-(1-l)\left(\tilde{q}_{1}-\tilde{q}_{2}\right)+2 a^{2}\left(\tilde{p}_{1}-\tilde{p}_{2}\right)\right|}{|x|}+ \\
& +\frac{C_{r}^{(3)}\left|\tilde{q}_{1}-\tilde{q}_{2}\right|}{\left|x^{1-\varepsilon}\right|}+\frac{C_{r}^{(4)}\left|\tilde{p}_{1}-\tilde{p}_{2}\right|}{\left|x^{1-\varepsilon}\right|},
\end{aligned}
$$

and

$$
\begin{aligned}
& \frac{1}{x} \mid 2 a^{2}\left\{\left[l-1-2 \tilde{q}_{2}\left(\tilde{p}_{1}+\tilde{p}_{2}\right)\right]\left(\tilde{p}_{1}-\tilde{p}_{2}\right)-2 \tilde{p}_{1}^{2}\left(\tilde{q}_{1}-\tilde{q}_{2}\right)\right\}+(l-1)\left\{\left[1-l+2 \tilde{p}_{1}\left(\tilde{q}_{1}+\tilde{q}_{2}\right)\right]\right. \\
& \left.\left(\tilde{q}_{1}-\tilde{q}_{2}\right)+2 \tilde{q}_{2}^{2}\left(\tilde{p}_{1}-\tilde{p}_{2}\right)\right\}\left|\leq \frac{C_{r}^{(5)}}{\left|x^{1-\varepsilon}\right|}\right| \tilde{q}_{1}-\tilde{q}_{2}\left|+\frac{C_{r}^{(6)}}{\left|x^{1-\varepsilon}\right|}\right| \tilde{p}_{1}-\tilde{p}_{2} \mid
\end{aligned}
$$

for some positive constants $C_{r}^{(3)}, \ldots, C_{r}^{(6)}$. Let us prove $(2.27)$ :

$$
\begin{aligned}
& \frac{\left|\left[1-l+2 \tilde{p}_{1}\left(\tilde{q}_{1}+\tilde{q}_{2}\right)\right]\left(\tilde{q}_{1}-\tilde{q}_{2}\right)+2 \tilde{q}_{2}^{2}\left(\tilde{p}_{1}-\tilde{p}_{2}\right)\right|}{|x|} \leq \frac{\left|-(1-l)\left(\tilde{q}_{1}-\tilde{q}_{2}\right)+2 a^{2}\left(\tilde{p}_{1}-\tilde{p}_{2}\right)\right|}{|x|}+ \\
& +\frac{\left|2 a g_{1}(x)+\frac{l-1}{a}\left(f_{1}(x)+f_{2}(x)\right)+x^{\varepsilon} g_{1}(x)\left(f_{1}(x)+f_{2}(x)\right)\right|}{\left|x^{1-\varepsilon}\right|}\left|\tilde{q}_{1}-\tilde{q}_{2}\right|+ \\
& +\frac{\left|2 f_{2}^{2}(x) x^{\varepsilon}+4 a f_{2}(x)\right|}{\left|x^{1-\varepsilon}\right|}\left|\tilde{p}_{1}-\tilde{p}_{2}\right| \leq \frac{\left|-(1-l)\left(\tilde{q}_{1}-\tilde{q}_{2}\right)+2 a^{2}\left(\tilde{p}_{1}-\tilde{p}_{2}\right)\right|}{|x|}+ \\
& +\frac{C_{r}^{(3)}}{\left|x^{1-\varepsilon}\right|}\left|\tilde{q}_{1}-\tilde{q}_{2}\right|+\frac{C_{r}^{(4)}}{\left|x^{1-\varepsilon}\right|}\left|\tilde{p}_{1}-\tilde{p}_{2}\right|,
\end{aligned}
$$

for some positive constants $C_{r}^{(3)}$ and $C_{r}^{(4)}$. The proof of $(2.28)$ is analogous. From the estimates (2.26), (2.27), (2.28), we obtain:

$$
\left(\begin{array}{c}
\left|\mathrm{Z}^{(1)^{\prime}}\right| \\
\left|\mathrm{Z}^{(2)^{\prime}}\right|
\end{array}\right) \leq\left(\frac{1}{|x|}\left(\begin{array}{ll}
0 & 1 \\
0 & 0
\end{array}\right)+\frac{\mathrm{A}_{1}}{\left|x^{1-l}\right|}+\frac{\mathrm{A}_{2}}{\left|x^{l}\right|}+\frac{\mathrm{A}_{3}}{\left|x^{1-\varepsilon}\right|}+\mathrm{A}_{4}\right)\left(\begin{array}{l}
\left|\mathrm{Z}^{(1)}\right| \\
\left|\mathrm{Z}^{(2)}\right|
\end{array}\right)
$$

for some constant matrices $\mathrm{A}_{1}, \mathrm{~A}_{2}, \mathrm{~A}_{3}$ and $\mathrm{A}_{4}$ (Here we mean $\leq$ component by component). Finally, choosing $\tilde{l}=\max \{1-\varepsilon, 1-l, l\}$, we obtain from $(2.29)$ :

$$
\left(\begin{array}{c}
D_{+, t} \mathrm{~V}^{(1)} \\
D_{+, t} \mathrm{~V}^{(2)}
\end{array}\right) \leq\left(\frac{1}{t}\left(\begin{array}{ll}
0 & 1 \\
0 & 0
\end{array}\right)+\frac{\tilde{\mathrm{A}}}{t^{\tilde{l}}}+\mathrm{A}_{4}\right)\left(\begin{array}{c}
\mathrm{V}^{(1)} \\
\mathrm{V}^{(2)}
\end{array}\right)
$$

where $\tilde{\mathrm{A}}=\mathrm{A}_{1}+\mathrm{A}_{2}+\mathrm{A}_{3}$ and $D_{+, t}$ is the right derivative w.r.t. $t$.

We perform the following change of variable $t^{1-\tilde{l}}=z$. The differential inequality for $\mathrm{V}$ in the new variable $z$ is

$$
D_{+, z} \mathrm{~V} \leq\left(\frac{1}{z}\left(\begin{array}{ll}
0 & 1 \\
0 & 0
\end{array}\right) \frac{1}{1-\tilde{l}}+\mathrm{A}(z)\right) \mathrm{V}, \quad \text { with } \quad \mathrm{A}(z)=\frac{\tilde{\mathrm{A}}}{1-\tilde{l}}+\frac{\mathrm{A}_{4}}{1-\tilde{l}} z^{\frac{\tilde{l}}{1-\tilde{l}}},
$$

where $D_{+, z}$ is the right derivative w.r.t. $z$. To show that $\mathrm{Z}=0$ we use the following: 
Comparison Theorem. Let us consider the following systems of $n$ first order ODEs in the real variable $z \in(0, a]$, for some $a>0$ :

$$
\begin{gathered}
D_{+} \mathrm{V}^{(i)} \leq F^{(i)}(z, \mathrm{~V}), \quad \mathrm{V}^{(i)}\left(x_{0}\right)=\mathrm{V}_{0}^{(i)}, \quad i=1, . ., n \\
\frac{\mathrm{dU}(i)}{\mathrm{d} z}=F^{(i)}(z, \mathrm{U}), \quad \mathrm{U}^{(i)}\left(x_{0}\right)=\mathrm{U}_{0}^{(i)}, \quad i=1, . ., n
\end{gathered}
$$

where $F^{(i)}(z, \mathrm{U})$ are continuous functions in $z \in(0, a],\left\|\mathrm{U}-\mathrm{U}_{0}\right\|<b$, non-decreasing in $\mathrm{U}^{(i)}$. If $\mathrm{V}_{0}^{(i)} \geq \mathrm{U}_{0}^{(i)}$, for $i=1, . . n$, then $\mathrm{V}^{(i)}(z) \geq \mathrm{U}^{(i)}(z)$, for every $0<z \leq z_{0}, i=1$,..n.

For the proof see [Lak].

We now apply Comparison Theorem to show that the assumption $Z\left(t_{0}\right) \neq 0$ for some $t_{0}>0$ leads to a contradiction. Observe that by definition $\tilde{l} \geq 1$, then $\mathrm{V}$ satisfies (2.31) with $\mathrm{V}_{0}^{(i)}>0$ and $F$ linear in $\mathrm{V}$ given by:

$$
F(z, \mathrm{~V})=\left(\frac{1}{z}\left(\begin{array}{ll}
0 & 1 \\
0 & 0
\end{array}\right) \frac{1}{1-\tilde{l}}+\frac{\tilde{\mathrm{A}}}{1-\tilde{l}}+\frac{\mathrm{A}_{4}}{1-\tilde{l}} z\right) \mathrm{V}
$$

By Comparison Theorem, for any solution of $(2.32)$ with $\mathrm{U}^{(i)}\left(z_{0}\right)=\mathrm{V}_{0}^{(i)}$, and $F(z, \mathrm{U})$ of the form (2.33), we have $\mathrm{V}^{(i)}(z) \geq \mathrm{U}^{(i)}(z)$, for every $0<z \leq z_{0}, i=1,2$. Moreover by standard arguments it is possible to take $\mathrm{U}$ in such a way that $\mathrm{U}^{(i)}(z) \geq 0$ and to continue the functions $\mathrm{U}, \mathrm{V}$ to $z=0$ preserving the relation:

$$
0 \leq \mathrm{U}^{(i)}(z) \leq \mathrm{V}^{(i)}(z)
$$

Thus, by (2.25) we obtain that U must satisfy

$$
\lim _{z \rightarrow 0} \frac{\mathrm{U}^{(i)}(z)}{z^{\frac{\varepsilon}{(1-i)}}}=0, \quad i=1,2 .
$$

Now, we use the following lemma:

Lemma 2.4. The only solution $\mathrm{U}$ of (2.32) with $F(z, \mathrm{U})$ given by (2.33) satisfying (2.34) is $\mathrm{U} \equiv 0$,

Proof. Any non-zero solution of $(2.32)$ with $F(z, \mathrm{U})$ of the form $(2.33)$ is given by

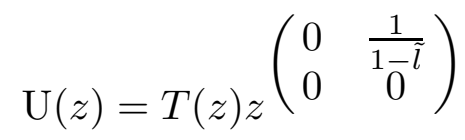

where $T(z)$ is a holomorphic matrix function, $T(z)=\left(\begin{array}{ll}1 & 0 \\ 0 & 1\end{array}\right)+\mathcal{O}(z)$. Now it is obvious that (2.35) does not satisfy (2.34). Thus $\mathrm{U} \equiv 0$, as we wanted to prove.

QED 
Using the above lemma, we obtain $\mathrm{U}_{0}^{(i)}=\mathrm{V}_{0}^{(i)}=0$, that contradicts the assumption $\mathrm{V}_{0}^{(i)}=\neq 0$. This concludes the proof of the uniqueness in the case $0<l<1$.

Let us briefly explain how to prove the uniqueness in the case $l=1$. Since the procedure is essentially the same as before, we shall skip the details. First of all we introduce the new variables $(\tilde{q}, \tilde{p})$ :

$$
\tilde{q}(x)=\frac{q(x)}{x} \sim a+x^{\varepsilon} f(x) \quad \tilde{p}(x)=p(x)-\mu(1-\mu) \sim x^{\varepsilon} g(x)
$$

which satisfy the equations of the motion:

$$
\begin{aligned}
& \dot{\tilde{q}}=Q_{1}(\tilde{q}, \tilde{p})+\frac{1}{x-1} Q_{2}(\tilde{q}, \tilde{p}) \\
& \dot{\tilde{p}}=-\frac{\tilde{p}}{x}+P_{1}(\tilde{q}, \tilde{p})+\frac{1}{x-1} P_{2}(\tilde{q}, \tilde{p})
\end{aligned}
$$

where $Q_{1}(\tilde{q}, \tilde{p})=2\left(\mu-\mu^{2}+\tilde{p}\right)(\tilde{q}-1) \tilde{q}^{2}, Q_{2}(\tilde{q}, \tilde{p})=\tilde{q}(\tilde{q}-1)[1+(2 \mu(1-\mu)+2 \tilde{p})(\tilde{q}-1)]$, $P_{1}(\tilde{q}, \tilde{p})=\left(\mu-\mu^{2}+\tilde{p}\right)^{2}(2-3 \tilde{q}) \tilde{q}-\mu(1-\mu)$, and $P_{2}(\tilde{q}, \tilde{p})=\tilde{p}+\left(\mu-\mu^{2}+\tilde{p}\right)^{2}\left(4 \tilde{q}-3 \tilde{q}^{2}-\right.$ 1) $-2\left(\mu-\mu^{2}+\tilde{p}\right) \tilde{q}$. Then, if we define $\mathrm{X}$ as before we obtain

$$
\mathrm{X}^{\prime}=\left(\begin{array}{c}
\Delta Q_{1}+\frac{\Delta Q_{2}}{x-1} \\
-\frac{\tilde{p}_{1}-\tilde{p}_{2}}{x}+\Delta P_{1}+\frac{\Delta P_{2}}{x-1}
\end{array}\right)
$$

that gives rise to the differential inequality:

$$
\left|\mathrm{X}^{\prime}\right| \leq\left(\left(\begin{array}{ll}
0 & 0 \\
0 & 1
\end{array}\right) \frac{1}{|x|}+\mathrm{A}_{1}+\frac{\mathrm{A}_{2}}{|x-1|}\right)|\mathrm{X}|
$$

for some constant matrices $\mathrm{A}_{1}$ and $\mathrm{A}_{2}$. Obviously $\mathrm{X}$ satisfies $(2.23)$ with any $0<\varepsilon<1$. Again we apply Comparison Theorem to $\mathrm{V}:=\left(\begin{array}{c}\left|\mathrm{X}^{(1)}\right| \\ \left|\mathrm{X}^{(2)}\right|\end{array}\right)$ along any fixed direction on the complex plane. We take $x$ such that $\arg (x)=\vartheta$ for some fixed $\vartheta$ and define $t=|x| . \mathrm{V}$ satisfies (2.31) with:

$$
F(t, \mathrm{~V})=\left(\frac{1}{t}\left(\begin{array}{ll}
0 & 0 \\
0 & 1
\end{array}\right)+\mathrm{A}_{1}+\frac{\mathrm{A}_{2}}{t-1}\right) \mathrm{V}
$$

If $\mathrm{V}_{0}^{(i)}>0$ then, thanks to Comparison Theorem, it is possible to take a solution $\mathrm{U}$ of $(2.32)$, with $\mathrm{U}_{0}^{(i)}=\mathrm{V}_{0}^{(i)}, i=1,2$, such that

$$
0 \leq \mathrm{U}^{(i)}(t) \leq \mathrm{V}^{(i)}(t)
$$

thus $\mathrm{U}^{(i)}$ satisfies (2.34). The general solution of (2.32) is

$$
\mathrm{U}=\mathrm{U}_{0}\left(\left(\begin{array}{ll}
1 & 0 \\
0 & t
\end{array}\right)+\mathcal{O}\left(t^{2}\right)\right)
$$

that satisfies (2.34) iff $\mathrm{U}_{0}=0$, namely $\mathrm{U} \equiv 0$ that is absurd. This concludes the proof of the uniqueness.

QED 
2.1.4. Asymptotic behaviour of the solutions of the Schlesinger equations. An important corollary to Theorem 2.2 is the following:

Theorem 2.3. The solutions of the Schlesinger equations $A_{0,1, x}(x)$ corresponding to the solution of Painlevé VI equation with asymptotic behaviour (2.15) must satisfy the relations (2.16) and (2.17).

Proof. Let us consider the solution $y(x)$ of Painlevé VI equation with asymptotic behaviour (2.15) and let us suppose that the corresponding solution of the Schlesinger equations $A_{0,1, x}(x)$ does not satisfy the relations (2.16) and (2.17). As shown in the lemma 2.2, for any constant matrices $A_{0,1, x}^{0}, \Lambda$ such that $\Lambda=A_{0}^{0}+A_{x}^{0}$ has eigenvalues $\pm \frac{\sigma}{2}, \sigma \in[0,1[$, and $A_{1}^{0}=-\Lambda-A_{\infty}$, there exists a solution $\hat{A}_{0,1, x}(x)$ of the Schlesinger equations that satisfy the relations (2.16) and (2.17). Now, as shown in Section 2.2, we can choose $A_{0,1, x}^{0}$ in order that the corresponding solution $\hat{y}(x)$ of Painlevé VI equation has exactly the asymptotic behaviour (2.15). Due to the uniqueness proved in Theorem 2.2, we have that $y(x)=\hat{y}(x)$, namely $A_{0,1, x}=\hat{A}_{0,1, x}$ up to conjugation by a constant diagonal matrix. This contradiction proves the theorem.

QED

2.1.5. Asymptotic behaviour of the $\mathbf{P V I} \mu$ solution near 1 and $\infty$. We now state the analogues of Theorem 2.2 for the local asymptotic behaviour of the solutions of (PVI) near the singular points $x=1, \infty$ :

Theorem 2.2'. For any pair of values $\left(a_{1}, \sigma_{1}\right), \sigma_{1} \in[0,1[$, there exists a unique branch of the solution of (PVI) with the asymptotic behaviour

$$
y(x) \sim 1-a_{1}(1-x)^{1-\sigma_{1}}\left(1+\mathcal{O}\left((1-x)^{\varepsilon}\right)\right) \quad \text { as } \quad x \rightarrow 1,
$$

for some $\varepsilon>0$.

The proof of this theorem is analogous to the proof of theorem 1, namely one can state the analogous of the lemma 2.2 replacing $x \mapsto 1-x$, and then choose suitably $\Lambda, A_{0,1, x}^{0}$. The uniqueness is proved in the same way as the case $x \mapsto 0$.

Theorem 2.2". For any pair of values $\left(a_{\infty}, \sigma_{\infty}\right), \sigma_{\infty} \in[0,1[$, there exists a unique branch of the solution of (PVI) with the asymptotic behaviour

$$
y(x) \sim a_{\infty} x^{\sigma_{\infty}}\left(1+\mathcal{O}\left(\left(x^{-\varepsilon}\right)\right) \text { as } x \rightarrow \infty,\right.
$$

for some $\varepsilon>0$.

The proof of uniqueness is analogous to the one of Theorem 2.2. The proof of existence follows the same strategy as the one of Theorem 2.2, but with a different formulation of the lemma 2.2:

Lemma 2.2'. Given some constant matrices $A_{i}^{0}, i=0,1, x$ with zero eigenvalues such that $\Lambda=A_{0}^{0}+A_{t}^{0}$ has eigenvalues $\pm \frac{\sigma}{2}, 0 \leq \sigma<1$, in any sector of $\overline{\mathbb{C}}$ containing none of the branch cuts, and sufficiently close to $\infty$, there exists a solution of the Schlesinger equations satisfying:

$$
\left|x^{A_{\infty}} A_{x}(x) x^{-A_{\infty}}-A_{1}^{0}\right| \leq K|x|^{\sigma^{\prime}-1} \quad\left|x^{\Lambda}\left(x^{A_{\infty}} A_{x}(x) x^{-A_{\infty}}-A_{1}^{0}\right) x^{-\Lambda}\right| \leq K|x|^{\sigma^{\prime}-1}
$$




$$
\left|x^{\Lambda} x^{A_{\infty}} A_{0,1}(x) x^{-A_{\infty}} x-^{\Lambda}-A_{x}^{0}\right| \leq K|x|^{\sigma^{\prime}-1},
$$

where $K$ is some positive constant and $1>\sigma^{\prime}>\sigma$.

Proof. Let us consider the Schlesinger equations (2.3) and perform the change of variable $x=\frac{1}{\hat{x}}$. Moreover we put:

$$
A_{i}(x):=x^{-A_{\infty}} \hat{A}_{i}(x) x^{A_{\infty}} ;
$$

Then we can apply Lemma 2.2 to the system:

$$
\begin{aligned}
\frac{\mathrm{d}}{\mathrm{d} \hat{x}} \hat{A}_{0}(\hat{x}) & =-\frac{\left[\hat{A}_{0}, \hat{A}_{1}\right]}{\hat{x}}, \\
\frac{\mathrm{d}}{\mathrm{d} \hat{x}} \hat{A}_{x}(\hat{x}) & =-\frac{\left[\hat{A}_{x}, \hat{A}_{1}\right]}{\hat{x}-1}, \\
\frac{\mathrm{d}}{\mathrm{d} \hat{x}} \hat{A}_{1}(\hat{x}) & =\frac{\left[\hat{A}_{0}, \hat{A}_{1}\right]}{\hat{x}}+\frac{\left[\hat{A}_{x}, \hat{A}_{1}\right]}{\hat{x}-1},
\end{aligned}
$$

and obtain the estimates (2.38) and (2.39).

QED

\subsection{The local asymptotic behaviour and the monodromy group of the Fuchsian system}

In this section we relate the local asymptotic behaviour of the solution $y(x)$ of $\mathrm{PVI} \mu$ to the monodromy data of the associated Fuchsian system (2.2). We essentially follow the same strategy of [Jim], even if we have to introduce some more tricks due to the fact that our matrices $A_{0,1, x}^{0}$ have eigenvalues all equal to zero. The main result of this section is the following:

Theorem 2.4. For the solution $y(x)$ of PVI $\mu$, such that $y(x) \sim a_{0} x^{1-\sigma_{0}}\left(1+\mathcal{O}\left(x^{\varepsilon}\right)\right)$, $0<\sigma_{0}<1$, the monodromy matrices of the Fuchsian system (2.2) have the form

$$
\begin{gathered}
M_{1}=\frac{-i}{\sin \pi \vartheta_{\infty}} \cdot \\
\cdot\left(\begin{array}{cc}
\cos \pi \sigma_{0}-e^{-i \pi \vartheta_{\infty}} & -2 e^{-i \pi \vartheta_{\infty}} \sin \frac{\pi\left(\vartheta_{\infty}+\sigma_{0}\right)}{2} \sin \frac{\pi\left(\vartheta_{\infty}-\sigma_{0}\right)}{2} \\
2 e^{i \pi \vartheta_{\infty}} \sin \frac{\pi\left(\vartheta_{\infty}+\sigma_{0}\right)}{2} \sin \frac{\pi\left(\vartheta_{\infty}-\sigma_{0}\right)}{2} & -\cos \pi \sigma_{0}+e^{i \pi \vartheta_{\infty}}
\end{array}\right) \\
C M_{x} C^{-1}=\frac{-i}{\sin \pi \sigma_{0}}\left(\begin{array}{cc}
e^{i \pi \sigma_{0}}-1 & 2 s e^{i \pi \sigma_{0}} \sin ^{2} \frac{\pi \sigma_{0}}{2} \\
-\frac{2}{s} e^{-i \pi \sigma_{0}} \sin ^{2} \frac{\pi \sigma_{0}}{2} & 1-e^{-i \pi \sigma_{0}}
\end{array}\right) \\
C M_{0} C^{-1}=\frac{-i}{\sin \pi \sigma_{0}}\left(\begin{array}{cc}
e^{i \pi \sigma_{0}}-1 & -2 s \sin ^{2} \frac{\pi \sigma_{0}}{2} \\
\frac{2}{s} \sin ^{2} \frac{\pi \sigma_{0}}{2} & 1-e^{-i \pi \sigma_{0}}
\end{array}\right)
\end{gathered}
$$

where $\vartheta_{\infty}=2 \mu$ and:

$$
\frac{s}{r}=\frac{1}{4 a_{0}} \frac{2 \mu+\sigma_{0}}{2 \mu-\sigma_{0}} \frac{\Gamma^{2}\left(1+\sigma_{0}\right) \Gamma^{2}\left(1-\frac{\sigma_{0}}{2}\right) \Gamma\left(1+\mu-\frac{\sigma_{0}}{2}\right) \Gamma\left(1-\mu-\frac{\sigma_{0}}{2}\right)}{\Gamma^{2}\left(1-\sigma_{0}\right) \Gamma^{2}\left(1+\frac{\sigma_{0}}{2}\right) \Gamma\left(1+\mu+\frac{\sigma_{0}}{2}\right) \Gamma\left(1-\mu+\frac{\sigma_{0}}{2}\right)}
$$


with an arbitrary complex number $r \neq 0$ and the matrix $C$ is:

$$
C=\left(\begin{array}{cc}
\sin \frac{\pi\left(\vartheta_{\infty}-\sigma_{0}\right)}{2} & r \sin \frac{\pi\left(\vartheta_{\infty}+\sigma_{0}\right)}{2} \\
\frac{1}{r} \sin \frac{\pi\left(\vartheta_{\infty}+\sigma_{0}\right)}{2} & \sin \frac{\pi\left(\vartheta_{\infty}-\sigma_{0}\right)}{2}
\end{array}\right) .
$$

In the case where $\sigma_{0}=0$ the monodromy matrices of the Fuchsian system (2.2) have the form

$$
\begin{gathered}
M_{1}=\frac{1}{\cos \frac{\pi \vartheta_{\infty}}{2}}\left(\begin{array}{cc}
e^{-i \pi \frac{\vartheta_{\infty}}{2}} & i \pi e^{-i \pi \frac{\vartheta}{2}} \\
-\frac{i}{\pi} \sin ^{2} \frac{\pi \vartheta_{\infty}}{2} e^{i \pi \frac{\vartheta}{2}} & e^{i \pi \frac{\vartheta_{\infty}}{2}}
\end{array}\right) \\
M_{0}=\left(\begin{array}{cc}
1-i s \tan \frac{\pi \vartheta_{\infty}}{2} & -i s \pi \exp \left(i \pi \frac{\vartheta_{\infty}}{2}\right) \sec \frac{\pi \vartheta_{\infty}}{2} \\
\frac{i}{\pi} s \sin ^{2} \frac{\pi \vartheta_{\infty}}{2} \exp \left(-i \pi \frac{\vartheta_{\infty}}{2}\right) \sec \frac{\pi \vartheta_{\infty}}{2} & 1+i s \tan \frac{\pi \vartheta_{\infty}}{2}
\end{array}\right), \\
M_{x}=\left(\begin{array}{cc}
1-i(1-s) \tan \frac{\pi \vartheta_{\infty}}{2} & -i(1-s) \pi \exp \left(i \pi \frac{\vartheta_{\infty}}{2}\right) \sec \frac{\pi \vartheta_{\infty}}{2} \\
\frac{i}{\pi}(1-s) \sin ^{2} \frac{\pi \vartheta_{\infty}}{2} \exp \left(-i \pi \frac{\vartheta_{\infty}}{2}\right) \sec \frac{\pi \vartheta_{\infty}}{2} & 1+i(1-s) \tan \frac{\pi \vartheta_{\infty}}{2}
\end{array}\right),
\end{gathered}
$$

where $s=a_{0}$.

The main idea to prove this theorem is that, due to Theorem 2.3, the solutions of the Schlesinger equations corresponding to the $\mathrm{PVI} \mu$ solution with the asymptotic behaviour (2.15) must satisfy the relations (2.16) and (2.17). Using these relations, we obtain the monodromy matrices of the Fuchsian system (2.2) via the ones of two simpler systems, given in the following two lemmas (see [SMJ] and [Jim]):

Lemma 2.5. Under the hypotheses (2.16), (2.17), the limit of the fundamental solution of the system (2.2), normalized at infinity, $\lim _{x \rightarrow 0} Y_{\infty}(z, x)=\hat{Y}(z)$, exists, for $z \in \overline{\mathbb{C}} \backslash\left\{B_{0} \cup\right.$ $\left.B_{x} \cup B_{1} \cup B_{\infty}\right\}, B_{0}, B_{x}, B_{1}$ and $B_{\infty}$ being balls around $0, x, 1$ and $\infty$ respectively. This limit $\hat{Y}$ satisfies the differential equation:

$$
\frac{\mathrm{d}}{\mathrm{d} z} \hat{Y}=\left(\frac{A_{1}^{0}}{z-1}+\frac{\Lambda}{z}\right) \hat{Y}
$$

and it has the following behaviour near the singularities of $(\hat{\Sigma})$

$$
\begin{aligned}
\hat{Y}(z)= & \left(1+\mathcal{O}\left(\frac{1}{z}\right)\right) z^{-A_{\infty}} \quad z \rightarrow \infty \\
& =(1+\mathcal{O}(z)) z^{\Lambda} \hat{C}_{0} \quad z \rightarrow 0 \\
& =\hat{G}_{1}(1+\mathcal{O}(z-1))(z-1)^{J_{1}} \hat{C}_{1} \quad z \rightarrow 1
\end{aligned}
$$

where $J_{1}$ is the Jordan normal forms of $A_{1}^{0}, \hat{G}_{1} J_{1} \hat{G}_{1}^{-1}=A_{1}^{0}, A_{\infty}=\left(\begin{array}{cc}\mu & 0 \\ 0 & -\mu\end{array}\right)$. Here $\hat{C}_{0}$, $\hat{C}_{1}$ are the connection matrices of the system $(\hat{\Sigma})$.

Remark 2.4. Observe that the matrix $\hat{C}_{0}$ is uniquely determined by the conditions (2.48). 
Lemma 2.6. Under the hypotheses (2.16), (2.17), the limit of the fundamental solution of the system (2.2), normalized around $\infty, \lim _{x \rightarrow 0} x^{-\Lambda} Y(x z, x)=\tilde{Y}(z) \hat{C}_{0}$ exists for $z \in$ $\overline{\mathbb{C}} \backslash\left\{B_{0} \cup B_{x} \cup B_{1} \cup B_{\infty}\right\}$. It satisfies the system

$$
\frac{\mathrm{d}}{\mathrm{d} z} \tilde{Y}=\left(\frac{A_{x}^{0}}{z-1}+\frac{A_{0}^{0}}{z}\right) \tilde{Y}
$$

and it has the following behaviour near the singularities of $(\tilde{\Sigma})$

$$
\begin{aligned}
\tilde{Y}(z)= & \left(1+\mathcal{O}\left(\frac{1}{z}\right)\right) z^{\Lambda} & & z \rightarrow \infty \\
& =\tilde{G}_{0}(1+\mathcal{O}(z)) z^{J_{0}} \tilde{C}_{0} & & z \rightarrow 0 \\
& =\tilde{G}_{1}(1+\mathcal{O}(z-1))(z-1)^{J_{1}} \tilde{C}_{1} & & z \rightarrow 1
\end{aligned}
$$

where $J_{0,1}$ are the Jordan normal forms of $A_{0, x}^{0}, \tilde{G}_{0,1}$ are such that $\tilde{G}_{0,1} J_{0,1} \tilde{G}_{0,1}^{-1}=A_{0, x}^{0}$. We denote $\tilde{C}_{0,1}$ the connection matrices of the system $(\tilde{\Sigma})$.

As we have seen above, the matrices of the two systems have the following form:

$$
A_{0}^{0}=\frac{1}{2} \Lambda+F, \quad A_{x}^{0}=\frac{1}{2} \Lambda-F, \quad A_{1}^{0}=-A_{\infty}-\Lambda,
$$

for some constant matrix $F$, and for $\Lambda$ and $T$ such that

$$
\Lambda=T\left(\begin{array}{cc}
\frac{\sigma}{2} & 0 \\
0 & -\frac{\sigma}{2}
\end{array}\right) T^{-1} .
$$

Using the relations (2.6), we have that

$$
F=T\left(\begin{array}{cc}
0 & \frac{b \sigma}{4} \\
\frac{-\sigma}{4 b} & 0
\end{array}\right) T^{-1}
$$

for some parameter $b$. As a consequence the systems $(\hat{\Sigma})$ and $(\tilde{\Sigma})$ are determined, up to diagonal conjugation, by the four entries of the matrix $T$ and by $b$.

Now, we explain how to compute the monodromy matrices of the original system (2.2) knowing the ones of the systems $(\hat{\Sigma})$ and $(\tilde{\Sigma})$. Later we will show how to compute the matrices $A_{0, x, 1}^{0}$ and the monodromy matrices of $(\hat{\Sigma})$ and $(\tilde{\Sigma})$.

Lemma 2.7. Let $\hat{M}_{0}, \hat{M}_{1}, \hat{M}_{\infty}=M_{\infty}$ be the monodromy matrices of the system $(\hat{\Sigma})$ with respect to the fundamental matrix $\hat{Y}$ and the basis $\hat{\gamma}_{0}=\gamma_{0} \gamma_{x}, \gamma_{1}$ in $\pi_{1}(\overline{\mathbb{C}} \backslash\{0,1, \infty\})$. Let $\tilde{M}_{0}, \tilde{M}_{1}, \tilde{M}_{\infty}=\exp (-2 \pi i \Lambda)$ be the monodromy matrices of the system $(\tilde{\Sigma})$ with respect to the fundamental matrix $\tilde{Y}$ and the basis $\tilde{\gamma}_{0}, \tilde{\gamma}_{1}=\gamma_{x}$. Then the monodromy matrices of the original system (2.2) are given by the formulae:

$$
M_{0}=\hat{C}_{0}^{-1} \tilde{M}_{0} \hat{C}_{0}, \quad M_{x}=\hat{C}_{0}^{-1} \tilde{M}_{1} \hat{C}_{0}, \quad M_{1}=\hat{M}_{1},
$$




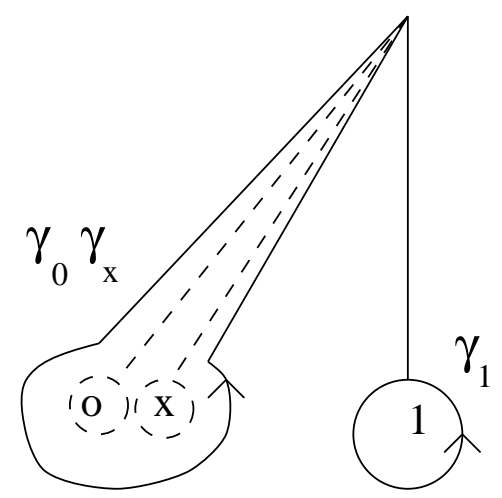

Fig.7. The paths $\gamma_{x}$ and $\gamma_{0}$ merge together as $x \rightarrow 0$. The homotopy class of $\gamma_{0} \gamma_{x}$ remains unchanged.

where $\hat{C}_{0}$ is defined by (2.48).

Proof. By the definition of $\hat{Y}$, the system $(\hat{\Sigma})$ is obtained by merging of the singularities 0 and $x$ of the system (2.2). We can choose the loop $\hat{\gamma}_{0}$ to be homotopic to $\gamma_{0} \gamma_{x}$, with $\hat{\gamma}_{0}$ not crossing a ball the $B_{0}$ (see figure 7 ).

As a consequence we obtain a relation between the monodromy matrices of the system (2.2) and the ones of the system $(\hat{\Sigma})$

$$
\begin{aligned}
\hat{M}_{\infty} & =M_{\infty}, \\
\hat{M}_{1} & =M_{1}, \\
\hat{M}_{0} & =M_{x} M_{0} .
\end{aligned}
$$

Similarly, by the definition of $\tilde{Y}$ the system $(\tilde{\Sigma})$ is obtained by the merging (see figure 8 ) of the singularities $z^{\prime}=\frac{1}{x}$ and $z^{\prime}=\infty$ of the system for $Y^{\prime}\left(z^{\prime}\right)$ :

$$
\frac{\mathrm{d}}{\mathrm{d} z^{\prime}} Y^{\prime}=\left(\frac{A_{0}}{z^{\prime}}+\frac{A_{1}}{z^{\prime}-\frac{1}{x}}+\frac{A_{x}}{z^{\prime}-1}\right) Y^{\prime}
$$



Fig.8. The paths $\gamma_{1}$ and $\gamma_{\infty}$ merge together as $x \rightarrow 0$. The homotopy class of $\tilde{\gamma} \equiv \gamma_{1} \gamma_{\infty}$ coincides with the one of $\left(\gamma_{0} \gamma_{x}\right)^{-1}$ 
So, in the basis $\hat{Y}$, the monodromy matrices of $(\tilde{\Sigma})$ have the following form:

$$
\begin{aligned}
\tilde{M}_{\infty} & =\hat{C}_{0}^{-1} M_{\infty} M_{1} \hat{C}_{0} \\
\tilde{M}_{1} & =\hat{C}_{0}^{-1} M_{x} \hat{C}_{0}, \\
\tilde{M}_{0} & =\hat{C}_{0}^{-1} M_{0} \hat{C}_{0} .
\end{aligned}
$$

The lemma is proved.

QED

Now we want to compute the monodromy matrices $\hat{M}_{i}$ and $\tilde{M}_{i}$ and the connection matrix $\hat{C}_{0}$. To this aim we have to solve the systems $(\hat{\Sigma})$ and $(\tilde{\Sigma})$, namely we have to determine $T$ and $b$. For $\sigma_{0} \neq 0$, this can be done introducing a suitable gauge transformation of $\hat{Y}$ and $\tilde{Y}$ such that the systems $(\hat{\Sigma})$ and $(\tilde{\Sigma})$ are equivalent to a Gauss equation. The case $\sigma_{0}=0$ will be treated later.

2.2.1. Reduction to the Gauss equation. First of all let us notice that both the systems $(\hat{\Sigma})$ and $(\tilde{\Sigma})$ have similar form. We want to reduce them, via a suitable gauge transformation and a appropriate choice of the parameters $\alpha, \beta, \gamma$, to systems of the form:

$$
\frac{\mathrm{d}}{\mathrm{d} z} Y(z, \alpha, \beta, \gamma)=\left(\frac{B_{0}}{z}+\frac{B_{1}}{z-1}\right) Y(z, \alpha, \beta, \gamma)
$$

where $B_{0}, B_{1}$ are some constant matrices with eigenvalues $1-\gamma, 0$ and $\gamma-\alpha-\beta-1,0$ respectively and $B_{0}+B_{1}=-\left(\begin{array}{cc}\alpha & 0 \\ 0 & \beta\end{array}\right)$.

Lemma 2.8. For $\alpha \neq \beta$, the system (2.52) is uniquely determined, up to a diagonal conjugation

$$
B_{0} \rightarrow T^{-1} B_{0} T, \quad B_{1} \rightarrow T^{-1} B_{1} T, \quad \text { with } \quad T=\left(\begin{array}{cc}
1 & 0 \\
0 & r
\end{array}\right), r \neq 0 .
$$

The entries $b_{i j}^{0}$ and $b_{i j}^{1}$ of the matrices $B_{0}$ and $B_{1}$ respectively, are given by the formulae

$$
\begin{aligned}
& b_{11}^{0}=\frac{\alpha(\gamma-1-\beta)}{\beta-\alpha}, \quad b_{22}^{0}=\frac{-\beta(\gamma-1-\alpha)}{\beta-\alpha}, \quad b_{11}^{1}=\frac{-\alpha(\gamma-1-\alpha)}{\beta-\alpha}, \\
& b_{22}^{1}=\frac{\beta(\gamma-1-\beta)}{\beta-\alpha}, \quad b_{12}^{0} b_{21}^{0}=b_{12}^{1} b_{21}^{1}=\frac{-\alpha \beta(\gamma-1-\beta)(\gamma-1-\alpha)}{(\beta-\alpha)^{2}} .
\end{aligned}
$$

The system (2.52) can be solved using the Gauss hypergeometric function. So, we can compute its connection matrices via the Kummer relations (see [Luke]) of the hypergeometric functions.

Lemma 2.9. The solutions of (2.52) have the form $Y=\left(\begin{array}{l}y_{1} \\ y_{2}\end{array}\right)$, with $y_{1}$ being an arbitrary solution of the following Gauss equation:

$$
z(1-z) y_{1}^{\prime \prime}+[c-(a+b+1) z] y_{1}^{\prime}-a b y_{1}=0
$$


where $a=\alpha, b=\beta+1, c=\gamma$ and $y_{2}$ given by:

$$
y_{2}(z)=r^{-1} \frac{\beta-\alpha}{\beta(\gamma-\beta-1)}\left\{z(z-1) \frac{\mathrm{d}}{\mathrm{d} z} y_{1}(z)+\left[\alpha z+\frac{\alpha(\gamma-\beta-1)}{\beta-\alpha}\right] y_{1}(z)\right\}
$$

where $r=-\frac{(\beta-\alpha) b_{12}^{0}}{\beta(\gamma-\beta-1)}$.

Proof. After the gauge transformation:

$$
Y(z, \alpha, \beta, \gamma)=z^{b_{11}^{0}}(1-z)^{b_{11}^{1}} U(z, \alpha, \beta, \gamma)
$$

one obtains from (2.52) the following Riemann equation for $u_{1}$

$$
u_{1}^{\prime \prime}+\left[\frac{1+b_{11}^{0}-b_{22}^{0}}{z}+\frac{1+b_{11}^{1}-b_{22}^{1}}{z-1}\right] u_{1}^{\prime}-\frac{b_{11}^{0} b_{22}^{0}}{z^{2}(z-1)^{2}} u_{1}=0 .
$$

Now $u_{1}$ is related with the solution $y_{G}$ of the Gauss equation (2.56), with $a=-b_{11}^{0}-b_{11}^{1}$, $b=1-b_{22}^{0}-b_{22}^{1}, c=1-b_{11}^{0}-b_{22}^{0}$, via the relation $u_{1}=z^{-b_{11}^{0}}(1-z)^{-b_{11}^{1}} y_{G}$. As a consequence, thanks to (2.54), (2.55), we obtain that $y_{1}=y_{G}$ and $a=\alpha+1, b=\beta, c=\gamma$. $y_{2}$ it is given by:

$$
\left(\frac{b_{12}^{0}}{z}+\frac{b_{12}^{1}}{z-1}\right) y_{2}=y_{1}^{\prime}-\left(\frac{b_{11}^{0}}{z}+\frac{b_{11}^{1}}{z-1}\right) y_{1}
$$

that gives the equation (2.57).

QED

To reduce the systems $(\hat{\Sigma})$ and $(\tilde{\Sigma})$ to the system $(2.52)$ we need to diagonalize the matrices $A_{1}^{0}+\Lambda=-A_{\infty}$ and $\Lambda$ respectively and to perform a suitable gauge transform. We need to introduce some notations. Denote $C_{0,1}^{\alpha, \beta, \gamma}$ the connection matrices of the system (2.52). The matrices $J_{0,1}$ are the Jordan normal forms of $B_{0,1}$ and the matrices $G_{0,1}^{\alpha, \beta, \gamma}$ are such that $G_{0,1}^{\alpha, \beta, \gamma} J_{0,1}\left(G_{0,1}^{\alpha, \beta, \gamma}\right)^{-1}=B_{0,1}$. Then for the asymptotic behaviour of an appropriate fundamental matrix $Y(z, \alpha, \beta, \gamma)$ of the system $(2.52)$ we have

$$
\begin{aligned}
& Y(z, \alpha, \beta, \gamma)=\left(1+\mathcal{O}\left(\frac{1}{z}\right)\right) z^{-\left(\begin{array}{ll}
\alpha & 0 \\
0 & \beta
\end{array}\right)} \\
& =G_{0}^{\alpha, \beta, \gamma}(1+\mathcal{O}(z)) z^{J_{0}} C_{0}^{\alpha, \beta, \gamma} \quad z \rightarrow 0 \\
& =G_{1}^{\alpha, \beta, \gamma}(1+\mathcal{O}(z-1))(z-1)^{J_{1}} C_{1}^{\alpha, \beta, \gamma} \quad z \rightarrow 1 \text {. }
\end{aligned}
$$

Some further remarks on the notations: from now on all the quantities with the hat are referred to the system $(\hat{\Sigma})$ and all the quantities with the tilde to the system $(\tilde{\Sigma})$. When we don't put any hat or tilde, the formulae are true for both the systems. In other words, they hold true for the generic system (2.52); substituting all the quantities with the correspondent hat or tilde ones, the formulae hold true for the systems $(\hat{\Sigma})$ or $(\tilde{\Sigma})$ respectively. 
We now choose the values of $\alpha, \beta, \gamma$ in relation with the eigenvalues of the matrices of the systems $(\hat{\Sigma})$ and $(\tilde{\Sigma})$. Namely, for $(\hat{\Sigma})$ we take

$$
\hat{\alpha}=\frac{\vartheta_{\infty}-\sigma_{0}}{2}, \quad \hat{\beta}=-\frac{\vartheta_{\infty}+\sigma_{0}}{2}, \quad \hat{\gamma}=1-\sigma_{0}
$$

and for $(\tilde{\Sigma})$ we take:

$$
\tilde{\alpha}=-\frac{\sigma_{0}}{2}, \quad \tilde{\beta}=\frac{\sigma_{0}}{2}, \quad \tilde{\gamma}=1 .
$$

With this choice of the values of $\alpha, \beta, \gamma$, one has:

$$
\hat{J}_{0}=\left(\begin{array}{cc}
1-\gamma & 0 \\
0 & 0
\end{array}\right), \quad \tilde{J}_{0}=\left(\begin{array}{ll}
0 & 1 \\
0 & 0
\end{array}\right), \quad \hat{J}_{1}=\tilde{J}_{1}=\left(\begin{array}{ll}
0 & 1 \\
0 & 0
\end{array}\right) \text {. }
$$

Now we can reduce the systems $(\hat{\Sigma})$ and $(\tilde{\Sigma})$ to the system $(2.52)$ via the following gauge transformations:

$$
\hat{Y}=z^{\frac{\hat{\alpha}+\hat{\beta}}{2}} Y(z, \hat{\alpha}, \hat{\beta}, \hat{\gamma}), \quad \tilde{Y}=G_{0}^{\hat{\alpha}, \hat{\beta}, \hat{\gamma}} Y(z, \tilde{\alpha}, \tilde{\beta}, \tilde{\gamma})
$$

where $G_{0}^{\hat{\alpha}, \hat{\beta}, \hat{\gamma}}$ is such that

$$
\Lambda-\frac{\hat{\alpha}+\hat{\beta}}{2} \operatorname{Id}=G_{0}^{\hat{\alpha}, \hat{\beta}, \hat{\gamma}}\left(\begin{array}{cc}
-\tilde{\alpha} & 0 \\
0 & -\tilde{\beta}
\end{array}\right)\left(G_{0}^{\hat{\alpha}, \hat{\beta}, \hat{\gamma}}\right)^{-1}
$$

As a consequence the connection matrices of $(2.52)$ are related to the ones of $(\hat{\Sigma})$ and $(\tilde{\Sigma})$ by the following formulae:

$$
\begin{gathered}
\hat{G}_{1}=G_{1}^{\hat{\alpha}, \hat{\beta}, \hat{\gamma}}, \quad \hat{C}_{1}=C_{1}^{\hat{\alpha}, \hat{\beta}, \hat{\gamma}}, \quad \hat{C}_{0}=G_{0}^{\hat{\alpha}, \hat{\beta}, \hat{\gamma}} C_{0}^{\hat{\alpha}, \hat{\beta}, \hat{\gamma}} \\
\tilde{G}_{0,1}=G_{0}^{\hat{\alpha}, \hat{\beta}, \hat{\gamma}} G_{0,1}^{\tilde{\alpha}, \tilde{\beta}, \tilde{\gamma}}, \quad \tilde{C}_{0,1}=C_{0,1}^{\tilde{\alpha}, \tilde{\beta}, \tilde{\gamma}}\left(G_{0}^{\hat{\alpha}, \hat{\beta}, \hat{\gamma}}\right)^{-1} .
\end{gathered}
$$

2.2.2. Local behaviour of the solution of (2.52). The solutions of (2.56) around the singular points $0,1, \infty$ are known and one can compute $y_{2}$ by (2.57). In this way one obtains the local behaviour of the fundamental solution $Y$ for $z \rightarrow 0,1, \infty$, and one can compute the connection matrices by the Kummer relations (which are the connection formulae for the hypergeometric equation). The difference w.r.t. the situation of [Jim] is that in our case the Gauss equation is degenerate, namely:

$$
\hat{c}-\hat{a}-\hat{b}=0, \quad \tilde{c}-\tilde{a}-\tilde{b}=0, \quad \tilde{c}=1 .
$$

So, we have to consider the logarithmic solutions of the Gauss equation around $z=1$ for both the systems $(\hat{\Sigma})$ and $(\tilde{\Sigma})$, and around $z=0$ for $(\tilde{\Sigma})$; moreover, we shall use the extension of the Kummer relations to this logarithmic case (see [Nor]). 


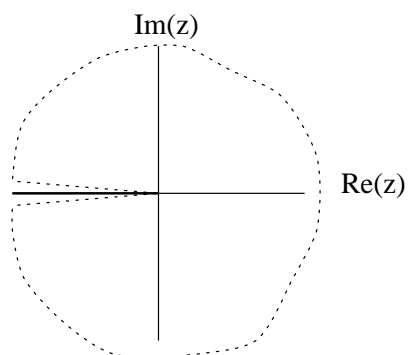

Fig.9. The branch cut $|\arg (z)|<\pi$.

In what follows we denote $F(a, b, c, z)$ the hypergeometric function and with $g(a, b, z)$ its logarithmic counterpart for $c=1$, namely:

$$
\begin{gathered}
F(a, b, c, z)=\sum_{k=0}^{\infty} \frac{(a)_{k}(b)_{k}}{k !(c)_{k}} z^{k}, \\
g(a, b, z)=\sum_{k=0}^{\infty} \frac{(a)_{k}(b)_{k}}{k !} z^{k}[\ln z+\psi(a+k)+\psi(b+k)-2 \psi(k+1)],
\end{gathered}
$$

with the branchcut $|\arg (z)|<\pi$ (see figure 9). Here $\psi$ is the logarithmic derivative of the gamma function, and the expressions of the parameters $a, b, c$ via $\alpha, \beta, \gamma$ are given in the lemma 2.9 .

Fundamental solution near $\infty$ Since $a-b \neq 0$, the solutions of (2.56) around $\infty$ are not logarithmic. We obtain

$$
Y_{\infty}=\left(\begin{array}{cc}
z^{-\alpha} F\left(\alpha,-\beta, \alpha-\beta, \frac{1}{z}\right) & \frac{-\alpha \beta z^{-\beta-1} r}{(\beta-\alpha)(\beta-\alpha+1)} F\left(\beta+1,1-\alpha, \beta-\alpha+2, \frac{1}{z}\right) \\
\frac{-\alpha \beta z^{-\alpha-1}}{r(\beta-\alpha)(\beta-\alpha-1)} F\left(\alpha+1,1-\beta, \alpha-\beta+2, \frac{1}{z}\right) & z^{-\beta} F\left(\beta, \alpha, \beta-\alpha, \frac{1}{z}\right)
\end{array}\right)
$$

The monodromy around $\infty$ is $\left(\begin{array}{cc}\exp (2 \pi i \alpha) & 0 \\ 0 & \exp (2 \pi i \beta)\end{array}\right)$.

Fundamental solution near 1 . Since $c-a-b=0$, the solutions are logarithmic:

$$
Y_{1}=\left(\begin{array}{cc}
F(\alpha, \beta+1,1,1-z) & \operatorname{rg}(\alpha, \beta+1,1,1-z) \\
\frac{1}{r} F(\alpha+1, \beta, 1,1-z) & g(\alpha+1, \beta, 1,1-z)
\end{array}\right)
$$

For $z \rightarrow 1$

$$
Y_{1} \sim G_{1}^{\alpha, \beta}(1-z)\left(\begin{array}{cc}
0 & 1 \\
0 & 0
\end{array}\right)
$$


with

$$
G_{1}^{\alpha, \beta}=\left(\begin{array}{cc}
1 & r[\psi(\alpha)+\psi(1+\beta)-2 \psi(1)] \\
\frac{1}{r} & \psi(1+\alpha)+\psi(\beta)-2 \psi(1)
\end{array}\right)
$$

The monodromy around 1 is $\left(\begin{array}{cc}1 & 2 i \pi r \\ 0 & 1\end{array}\right)$.

Fundamental solution near 0 . We have to distinguish the case $(\hat{\Sigma})$, where the solutions of (2.56) around 0 are not logarithmic, and the case $(\tilde{\Sigma})$, where $c=1$ and the solutions are logarithmic.

For $(\hat{\Sigma})$ one has

$$
\hat{Y}_{0}=\left(\begin{array}{cc}
-\frac{\hat{\alpha}}{\hat{\beta}-\hat{\alpha}} z^{-\hat{\alpha}-\hat{\beta}} F(-\hat{\beta}, 1-\hat{\alpha}, 1-\hat{\alpha}-\hat{\beta}, z) & \hat{r} \frac{\hat{\beta}}{\hat{\beta}-\hat{\alpha}} F(\hat{\alpha}, \hat{\beta}+1, \hat{\alpha}+\hat{\beta}+1, z) \\
-\frac{\hat{\beta}}{\hat{r}(\hat{\beta}-\hat{\alpha})} z^{-\hat{\alpha}-\hat{\beta}} F(1-\hat{\beta},-\hat{\alpha}, 1-\hat{\alpha}-\hat{\beta}, z) & \frac{\hat{\alpha}}{\hat{\beta}-\hat{\alpha}} F(\hat{\alpha}+1, \hat{\beta}, \hat{\alpha}+\hat{\beta}+1, z)
\end{array}\right) .
$$

For $z \rightarrow 0$ it behaves like

$$
\hat{Y}_{0} \sim G_{0}^{\hat{\alpha}, \hat{\beta}} z\left(\begin{array}{cc}
-\hat{\alpha}-\hat{\beta} & 0 \\
0 & 0
\end{array}\right)
$$

where

$$
G_{0}^{\hat{\alpha}, \hat{\beta}}=\frac{1}{\hat{\beta}-\hat{\alpha}}\left(\begin{array}{cc}
-\hat{\alpha} & \hat{r} \hat{\beta} \\
-\frac{\hat{\beta}}{\hat{r}} & \hat{\alpha}
\end{array}\right) .
$$

The monodromy around 0 is $\left(\begin{array}{cc}\exp (-2 i \pi(\hat{\alpha}+\hat{\beta}) & 0 \\ 0 & 1\end{array}\right)$.

For $(\tilde{\Sigma})$ one has

$$
\tilde{Y}_{0}=\left(\begin{array}{cc}
F(\tilde{\alpha}, 1-\tilde{\alpha}, 1, z) & \tilde{r} g(\tilde{\alpha}, 1-\tilde{\alpha}, 1, z) \\
-\frac{1}{\tilde{r}} F(\tilde{\alpha}+1,-\tilde{\alpha}, 1, z) & -g(\tilde{\alpha}+1,-\tilde{\alpha}, 1, z)
\end{array}\right),
$$

for $z \rightarrow 0$ it behaves like

$$
\tilde{Y}_{0} \sim G_{0}^{\tilde{\alpha}} z\left(\begin{array}{ll}
0 & 1 \\
0 & 0
\end{array}\right)
$$

with

$$
G_{0}^{\tilde{\alpha}}=\left(\begin{array}{cc}
1 & \tilde{r}[\psi(1-\tilde{\alpha})+\psi(\tilde{\alpha})-2 \psi(1)] \\
-\frac{1}{\tilde{r}} & -\psi(1+\tilde{\alpha})-\psi(-\tilde{\alpha})+2 \psi(1)
\end{array}\right) .
$$

The monodromy around 0 is $\left(\begin{array}{cc}1 & 2 i \pi \tilde{r} \\ 0 & 1\end{array}\right)$.

2.2.3. Connection formulae. In order to compute the connection matrices we write $Y_{\infty}$ in the form:

$$
Y_{\infty}=\left(\begin{array}{lrr}
\exp (-i \pi \alpha) U(\alpha, \beta+1, z) & \frac{-\alpha \beta \exp (-i \pi(\beta+1)) r}{(\beta-\alpha)(\beta-\alpha+1)} U(\beta+1, \alpha, z) \\
\frac{-\alpha \beta \exp (-i \pi(\alpha+1))}{r(\beta-\alpha)(\beta-\alpha-1)} U(\alpha+1, \beta, z) & \exp (-i \pi \beta) U(\beta, \alpha+1, z)
\end{array}\right)
$$


where $U(a, b, z):=\left(z^{-1} e^{i \pi}\right)^{-a} F\left(a, 1-b, 1+a-b, \frac{1}{z}\right)$. For $z$ such that $|\arg (z)|<2 \pi$, there are the following connection formulae:

$$
\begin{gathered}
\left.U(a, b, z)\right|_{z \rightarrow 1}=\frac{-\exp (i \pi a) \Gamma(1+a-b)}{\Gamma(a) \Gamma(1-b)}\{[i \pi+\psi(1-b)-\psi(b)] F(a, b, 1,1-z)+ \\
g(a, b, 1,1-z)\}, \\
\left.U(a, 1-a, z)\right|_{z \rightarrow 0}=\frac{-\Gamma(2 a)}{\Gamma(a)^{2}}\{[-i \pi+\psi(a)-\psi(1-a)] F(a, 1-a, 1, z)+g(a, 1-a, 1, z)\}, \\
\left.U(a, b, z)\right|_{z \rightarrow 0}=\frac{\Gamma(1+a-b) \Gamma(1-a-b)}{\Gamma(1-b)^{2}} F(a, b, a+b, z)+\frac{\Gamma(1+a-b) \Gamma(a+b-1)}{\Gamma(a)^{2}} . \\
\cdot z^{1-a-b} F(1-b, 1-a, 2-a-b, z) .
\end{gathered}
$$

Using these relations we obtain the analytic continuation of $Y_{\infty}$ around 0 and 1 , and by the definition of the connection matrices

$$
\left.Y_{\infty}\right|_{z \rightarrow 0,1}=Y_{0,1} C_{0,1}^{\alpha, \beta, \gamma}
$$

we obtain, by straightforward computations

$$
\begin{aligned}
& C_{0}^{\hat{\alpha}, \hat{\beta}}=\left(\begin{array}{cc}
\frac{\exp (i \pi \hat{\beta}) \Gamma(\hat{\alpha}+\hat{\beta}) \Gamma(\hat{\alpha}-\hat{\beta}+1)}{\Gamma(\hat{\alpha}) \Gamma(1+\hat{\alpha})} & -\hat{r} \frac{\exp (-i \pi \hat{\alpha}) \Gamma(\hat{\alpha}+\hat{\beta}) \Gamma(1-\hat{\alpha}+\hat{\beta})}{\Gamma(\hat{\beta}) \Gamma(1+\hat{\beta})} \\
\frac{1}{\hat{r}} \frac{\exp (-i \pi \hat{\alpha}) \Gamma(-\hat{\alpha}-\hat{\beta}) \Gamma(\hat{\alpha}-\hat{\beta}+1)}{\Gamma(1-\hat{\beta}) \Gamma(-\hat{\beta})} & -\frac{\exp (-i \pi \hat{\beta}) \Gamma(-\hat{\alpha}-\hat{\beta}) \Gamma(1-\hat{\alpha}+\hat{\beta})}{\Gamma(-\hat{\alpha}) \Gamma(1-\hat{\alpha})}
\end{array}\right) \\
& C_{1}^{\hat{\alpha}, \hat{\beta}}=\left(\begin{array}{cc}
-\frac{\Gamma(\hat{\alpha}-\hat{\beta})}{\Gamma(\hat{\alpha}) \Gamma(-\hat{\beta})}[i \pi+\pi \cot (\pi \hat{\alpha})] & -\frac{\Gamma(-\hat{\alpha}+\hat{\beta})}{\Gamma(-\hat{\alpha}) \Gamma(\hat{\beta})}[i \pi+\pi \cot (\pi \hat{\beta})] \\
-\frac{1}{\hat{r}} \frac{\Gamma(\hat{\alpha}-\hat{\beta})}{\Gamma(\hat{\alpha}) \Gamma(-\hat{\beta})} & -\frac{\Gamma(-\hat{\alpha}+\hat{\beta})}{\Gamma(-\hat{\alpha}) \Gamma(\hat{\beta})}
\end{array}\right), \\
& C_{0}^{\tilde{\alpha}}=\left(\begin{array}{cc}
\frac{\Gamma(2 \tilde{\alpha})}{\Gamma^{2}(\tilde{\alpha})} \exp (-i \pi \tilde{\alpha})[i \pi+\pi \cot (\pi \tilde{\alpha})] & \tilde{r} \frac{\Gamma(-2 \tilde{\alpha})}{\Gamma^{2}(-\tilde{\alpha})} \exp (i \pi \tilde{\alpha})[\pi \cot (\pi \hat{\alpha})-i \pi] \\
-\frac{1}{\tilde{r}} \frac{\Gamma(2 \tilde{\alpha})}{\Gamma^{2}(\tilde{\alpha})} \exp (-i \pi \tilde{\alpha}) & \frac{\Gamma(-2 \tilde{\alpha})}{\Gamma^{2}(-\tilde{\alpha})} \exp (i \pi \tilde{\alpha})
\end{array}\right) \\
& C_{1}^{\tilde{\alpha}}=\left(\begin{array}{cc}
-\frac{\Gamma(2 \tilde{\alpha})}{\Gamma^{2}(\tilde{\alpha})}[i \pi-\pi \cot (\pi \tilde{\alpha})] & -\tilde{r} \frac{\Gamma(-2 \tilde{\alpha})}{\Gamma^{2}(-\tilde{\alpha})}[\pi \cot (\pi \hat{\alpha})+i \pi] \\
-\frac{1}{\tilde{r}} \frac{\Gamma(2 \tilde{\alpha})}{\Gamma^{2}(\tilde{\alpha})} & -\frac{\Gamma(-2 \tilde{\alpha})}{\Gamma^{2}(-\tilde{\alpha})}
\end{array}\right) .
\end{aligned}
$$

Now we have to compute the monodromy matrices in the basis $Y_{\infty}$. Using the formulae (2.51), (2.61) and (2.62) we have

$$
M_{1}=\left(C_{1}^{\hat{\alpha}, \hat{\beta}}\right)^{-1}\left(\begin{array}{cc}
1 & 2 \pi i \hat{r} \\
0 & 1
\end{array}\right) C_{1}^{\hat{\alpha}, \hat{\beta}}, \quad M_{0, x}=\left(C_{0}^{\hat{\alpha}, \hat{\beta}}\right)^{-1}\left(C_{0,1}^{\tilde{\alpha}}\right)^{-1}\left(\begin{array}{cc}
1 & 2 \pi i \tilde{r} \\
0 & 1
\end{array}\right) C_{0,1}^{\tilde{\alpha}} C_{0}^{\hat{\alpha}, \hat{\beta}}
$$

Now we put

$$
\hat{r}=\frac{-\exp [-i \pi(\hat{\alpha}-\hat{\beta})] \Gamma(\hat{\alpha}-\hat{\beta}) \Gamma(\hat{\beta}) \Gamma(-\hat{\alpha})}{\Gamma(\hat{\beta}-\hat{\alpha}) \Gamma(-\hat{\beta}) \Gamma(\hat{\alpha})} r
$$


and

$$
\tilde{r}=\frac{\exp (-2 i \pi \tilde{\alpha}) \Gamma(2 \tilde{\alpha}) \Gamma(-\tilde{\alpha})^{2}}{\Gamma(-2 \tilde{\alpha}) \Gamma(\tilde{\alpha})^{2}} \tilde{s}
$$

In this way we immediately obtain the formula (2.40) for $M_{1}$ and it turns out that

$$
C_{0}^{\hat{\alpha}, \hat{\beta}}=D^{\hat{\alpha}, \hat{\beta}} \cdot C
$$

where $C$ is given in the formula $(2.44)$ and

$$
D^{\hat{\alpha}, \hat{\beta}}:=\left(\begin{array}{cc}
\frac{\exp (i \pi \beta) \Gamma(\hat{\beta}+\hat{\alpha}) \Gamma(1-\hat{\beta}+\hat{\alpha})}{\hat{\alpha} \Gamma(\hat{\alpha})^{2} \sin \pi \hat{\alpha}} & 0 \\
0 & \frac{-\exp (-i \pi \beta) \Gamma(-\hat{\beta}-\hat{\alpha}) \Gamma(1+\hat{\beta}-\hat{\alpha})}{\Gamma(1-\hat{\alpha}) \Gamma(-\hat{\alpha}) \sin \pi \hat{\alpha}}
\end{array}\right) .
$$

As a consequence, one has

$$
C M_{0, x} C^{-1}=\left(D^{\hat{\alpha}, \hat{\beta}}\right)^{-1}\left(C_{0,1}^{\tilde{\alpha}}\right)^{-1}\left(\begin{array}{cc}
1 & 2 \pi i \tilde{r} \\
0 & 1
\end{array}\right) C_{0,1}^{\tilde{\alpha}} D^{\hat{\alpha}, \hat{\beta}}
$$

By straightforward computations one can easily check that, for

$$
\tilde{s}=-\frac{\exp (2 i \pi \beta) \Gamma(\hat{\beta}+\hat{\alpha}) \Gamma(1-\hat{\beta}+\hat{\alpha}) \Gamma(1-\hat{\alpha}) \Gamma(-\hat{\alpha})}{\Gamma(-\hat{\beta}-\hat{\alpha}) \Gamma(1+\hat{\beta}-\hat{\alpha}) \hat{\alpha} \Gamma(\hat{\alpha})^{2}} s
$$

i.e. for

$$
\frac{\tilde{r}}{\hat{r}}=-\frac{\Gamma\left(1-\sigma_{0}\right)^{2} \Gamma\left(\frac{\sigma_{0}}{2}\right)^{2}}{\Gamma\left(1+\sigma_{0}\right)^{2} \Gamma\left(-\frac{\sigma_{0}}{2}\right)^{2}} \frac{\Gamma\left(1+\frac{\vartheta_{\infty}+\sigma_{0}}{2}\right) \Gamma\left(1+\frac{-\vartheta_{\infty}+\sigma_{0}}{2}\right)}{\Gamma\left(1+\frac{\vartheta_{\infty}-\sigma_{0}}{2}\right) \Gamma\left(1-\frac{\vartheta_{\infty}+\sigma_{0}}{2}\right)} \frac{s}{r},
$$

the formulae (2.41), (2.42) hold true.

To conclude the proof we have to prove the relation (2.43), namely we want to prove that $\frac{\tilde{r}}{\hat{r}}=-\frac{1}{4 a_{0}} \frac{2 \mu+\sigma_{0}}{2 \mu-\sigma_{0}}$. To this aim we compute the matrices $A_{0,1, x}^{0}$ and $\Lambda$ and then the asymptotic behaviour of $y$ in terms of $\sigma_{0}$ and $\tilde{r}$. To compute the matrices $A_{0,1, x}^{0}$ and $\Lambda$ we observe that, thanks to the gauges (2.60),

$$
A_{1}^{0}=\hat{B}_{1}, \quad \Lambda=\hat{B}_{0}+\frac{\hat{\alpha}+\hat{\beta}}{2} \mathbf{1}, \quad A_{0, x}^{0}=G_{0}^{\hat{\alpha}, \hat{\beta}} \tilde{B}_{0,1}\left(G_{0}^{\hat{\alpha}, \hat{\beta}}\right)^{-1}
$$

First of all one has to compute the $B_{0,1}$ :

$$
B_{0}=G_{0}^{\alpha, \beta, \gamma} J_{0}\left(G_{0}^{\alpha, \beta, \gamma}\right)^{-1}, \quad B_{1}=G_{1}^{\alpha, \beta, \gamma} J_{1}\left(G_{1}^{\alpha, \beta, \gamma}\right)^{-1}
$$

then

$$
\hat{B}_{0}=\frac{1}{\hat{\beta}-\hat{\alpha}}\left(\begin{array}{cc}
\alpha^{2} & -\hat{r} \hat{\alpha} \beta \\
\frac{\hat{\alpha} \beta}{\hat{r}} & -\hat{\beta}^{2}
\end{array}\right), \quad \hat{B}_{1}=\frac{\hat{\alpha} \hat{\beta}}{\hat{\beta}-\hat{\alpha}}\left(\begin{array}{cc}
-1 & \hat{r} \\
-\frac{1}{\hat{r}} & 1
\end{array}\right),
$$

and

$$
\tilde{B}_{1}=\frac{\tilde{\alpha}}{2}\left(\begin{array}{ll}
-1 & \tilde{r} \\
-\frac{1}{\tilde{r}} & 1
\end{array}\right), \quad \tilde{B}_{0}=-\frac{\tilde{\alpha}}{2}\left(\begin{array}{cc}
1 & \tilde{r} \\
-\frac{1}{\tilde{r}} & -1
\end{array}\right)
$$


It is then obvious that, referring to $(2.49)$ and $(2.50), b=\tilde{r}, T=G_{0}^{\hat{\alpha}, \hat{\beta}}$. Using the formula (2.18),

$$
y(x) \sim-\frac{\hat{r}\left(\sigma_{0}+2 \mu\right)}{4 \tilde{r}\left(2 \mu-\sigma_{0}\right)} x^{1-\sigma_{0}} .
$$

This proves the formula (2.43) and concludes the proof of the theorem, in the case $\sigma_{0} \neq 0$.

For completeness we write here the result for the matrices $A_{0,1, x}^{0}$ and $\Lambda$ :

$$
\begin{aligned}
& \Lambda=\frac{1}{4 \vartheta_{\infty}}\left(\begin{array}{cc}
-\vartheta_{\infty}^{2}-\sigma_{0}^{2} & \left(-\vartheta_{\infty}^{2}+\sigma_{0}^{2}\right) \hat{r} \\
\frac{-\sigma_{0}^{2}+\vartheta_{\infty}^{2}}{\hat{r}} & \vartheta_{\infty}^{2}+\sigma_{0}^{2}
\end{array}\right), \quad A_{1}^{0}=\frac{\vartheta_{\infty}^{2}-\sigma_{0}^{2}}{4 \vartheta_{\infty}}\left(\begin{array}{cc}
-1 & \hat{r} \\
-\frac{1}{\hat{r}} & 1
\end{array}\right), \\
& A_{0}^{0}=\frac{1}{8 \theta}\left(\begin{array}{l}
\frac{\theta^{2}-\sigma_{0}^{2}}{2}\left(\frac{\tilde{r}}{\hat{r}}+\frac{\hat{r}}{\tilde{r}}\right)-\theta^{2}-\sigma_{0}^{2} \\
\frac{1}{\hat{r}}\left(-\frac{\left(\theta-\sigma_{0}\right)^{2} \hat{r}}{2 \tilde{r}}-\frac{\left(\theta+\sigma_{0}\right)^{2} \tilde{r}}{2 \hat{r}}+\theta^{2}-\sigma_{0}^{2}\right.
\end{array}\right) \\
& \hat{r}\left(\frac{\left(\theta-\sigma_{0}\right)^{2} \tilde{r}}{2 \hat{r}}+\frac{\left(\theta+\sigma_{0}\right)^{2} \hat{r}}{2 \tilde{r}}+\sigma_{0}^{2}-\theta^{2}\right) \\
& \left.-\frac{\theta^{2}-\sigma_{0}^{2}}{2}\left(\frac{\tilde{r}}{\hat{r}}+\frac{\hat{r}}{\tilde{r}}\right)+\theta^{2}+\sigma_{0}^{2}\right) \\
& A_{x}^{0}=\frac{1}{8 \vartheta_{\infty}}\left(\begin{array}{l}
\frac{\sigma_{0}^{2}-\vartheta_{\infty}^{2}}{2}\left(\frac{\tilde{r}}{\hat{r}}+\frac{\hat{r}}{\tilde{r}}\right)-\vartheta_{\infty}^{2}-\sigma_{0}^{2} \\
\frac{1}{\hat{r}}\left(\frac{\left(\vartheta_{\infty}-\sigma_{0}\right)^{2} \hat{r}}{2 \tilde{r}}+\frac{\left(\vartheta_{\infty}+\sigma_{0}\right)^{2} \tilde{r}}{2 \hat{r}}+\vartheta_{\infty}{ }^{2}-\sigma_{0}^{2}\right.
\end{array}\right) \\
& \left.\begin{array}{r}
\hat{r}\left(-\frac{\left(\vartheta_{\infty}-\sigma_{0}\right)^{2} \tilde{r}}{2 \hat{r}}-\frac{\left(\vartheta_{\infty}+\sigma_{0}\right)^{2} \hat{r}}{2 \tilde{r}}+\sigma_{0}^{2}-\vartheta_{\infty}^{2}\right) \\
\frac{\vartheta_{\infty}^{2}-\sigma_{0}^{2}}{2}\left(\frac{\tilde{r}}{\hat{r}}+\frac{\hat{r}}{\tilde{r}}\right)+\vartheta_{\infty}^{2}+\sigma_{0}^{2}
\end{array}\right) .
\end{aligned}
$$

2.2.4. Case $\sigma_{0}=0$. In this case the solution of the system $(\hat{\Sigma})$ has logarithmic behaviour around 0. Moreover, as seen before, it has a logarithmic behaviour around 1. For this system we can use all the formulae derived for $(\tilde{\Sigma})$, substituting $\tilde{\alpha}$ by $\hat{\alpha}$. The treatment of the $(\tilde{\Sigma})$, is even easier. Ideed in this case $\Lambda$ has zero eigenvalues and it is straightforward to solve the system (2.52) exactly. In fact in this case we have

$$
\tilde{B}_{0}+\tilde{B}_{1}=\left(\begin{array}{ll}
0 & 1 \\
0 & 0
\end{array}\right), \quad \operatorname{det} \tilde{B}_{i}=\operatorname{Tr} \tilde{B}_{i}=0, \quad i=0,1 .
$$

Then the matrices $\tilde{B}_{0}$ and $\tilde{B}_{1}$ are uniquely determined up to an arbitrary parameter $s$ :

$$
\tilde{B}_{0}=\left(\begin{array}{cc}
0 & s \\
0 & 0
\end{array}\right), \quad \tilde{B}_{1}=\left(\begin{array}{cc}
0 & 1-s \\
0 & 0
\end{array}\right)
$$

and we can solve the differential equation (2.52) explicitly:

$$
\tilde{Y}=\left(\begin{array}{cc}
1 & s \log z+(1-s) \log (z-1) \\
0 & 1
\end{array}\right)
$$


The solution $\tilde{Y}$ has the following asymptotic behaviour near the singular points:

$$
\begin{aligned}
\tilde{Y} & =\left(\mathbf{1}+\mathcal{O}\left(\frac{1}{z}\right)\right) z^{J}, \quad \text { as } \quad z \rightarrow \infty \\
& =\tilde{G}_{0}(\mathbf{1}+\mathcal{O}(z)) z^{J} \tilde{C}_{0}, \quad \text { as } \quad z \rightarrow 0, \\
& =\tilde{G}_{1}(\mathbf{1}+\mathcal{O}(z-1))(z-1)^{J} \tilde{C}_{1} \quad \text { as } \quad z \rightarrow 1,
\end{aligned}
$$

where $J=\left(\begin{array}{ll}0 & 1 \\ 0 & 0\end{array}\right)$. It's easy to verify that

$$
\tilde{C}_{0}=\left(\begin{array}{cc}
1 & 0 \\
0 & s
\end{array}\right), \quad \tilde{C}_{1}=\left(\begin{array}{cc}
1 & 0 \\
0 & 1-s
\end{array}\right), \quad \tilde{G}_{0}=\left(\begin{array}{cc}
1 & 0 \\
0 & \frac{1}{s}
\end{array}\right), \quad \tilde{G}_{1}=\left(\begin{array}{cc}
1 & 0 \\
0 & \frac{1}{1-s}
\end{array}\right) .
$$

As a consequence the monodromy matrices of the system (2.52) are

$$
\tilde{M}_{0}=\left(\begin{array}{cc}
1 & 2 \pi i s \\
0 & 1
\end{array}\right), \quad \tilde{M}_{1}=\left(\begin{array}{cc}
1 & 2 \pi i(1-s) \\
0 & 1
\end{array}\right)
$$

The correspondent monodromy matrices of the full system (2.2) are given by:

$$
M_{1}=\left(C_{1}^{\hat{\alpha}}\right)^{-1}\left(\begin{array}{cc}
1 & 2 i \pi \hat{r} \\
0 & 1
\end{array}\right) C_{1}^{\hat{\alpha}}, \quad \text { and } \quad M_{0, x}=\left(C_{0}^{\hat{\alpha}}\right)^{-1} \tilde{M}_{0,1} C_{0}^{\hat{\alpha}},
$$

where

$$
C_{0}^{\hat{\alpha}}=\left(\begin{array}{cc}
\frac{\pi \Gamma(2 \hat{\alpha})}{\Gamma^{2}(\hat{\alpha}) \sin \pi \hat{\alpha}} & \hat{r} \frac{\pi \Gamma(-2 \hat{\alpha})}{\Gamma^{2}(-\hat{\alpha}) \sin \pi \hat{\alpha}} \\
-\frac{1}{\hat{r}} \frac{\Gamma(2 \hat{\alpha}) \exp (-i \pi \hat{\alpha})}{\Gamma^{2}(\hat{\alpha})} & \frac{\Gamma(-2 \hat{\alpha}) \exp (i \pi \hat{\alpha})}{\Gamma^{2}(-\hat{\alpha})}
\end{array}\right)
$$

and

$$
C_{1}^{\hat{\alpha}}=\left(\begin{array}{cc}
\frac{\pi \Gamma(2 \hat{\alpha}) \exp (-i \pi \hat{\alpha})}{\Gamma^{2}(\hat{\alpha}) \sin \pi \hat{\alpha}} & -\hat{r} \frac{\pi \Gamma(-2 \hat{\alpha}) \exp (i \pi \hat{\alpha})}{\Gamma^{2}(-\hat{\alpha}) \sin \pi \hat{\alpha}} \\
-\frac{1}{\hat{r}} \frac{\Gamma(2 \hat{\alpha})}{\Gamma^{2}(\hat{\alpha})} & -\frac{\Gamma(-2 \hat{\alpha})}{\Gamma^{2}(-\hat{\alpha})}
\end{array}\right)
$$

We observe that

$$
\hat{C}_{0,1}^{\hat{\alpha}}=C^{0,1} D^{\hat{\alpha}}
$$

where

$$
D^{\hat{\alpha}}=\left(\begin{array}{cc}
\frac{\pi \Gamma(2 \hat{\alpha}) \exp (-i \pi \hat{\alpha})}{\Gamma(\hat{\alpha})^{2} \sin \pi \hat{\alpha}} & 0 \\
0 & \frac{-\Gamma(-2 \hat{\alpha})}{\Gamma(-\hat{\alpha})^{2}}
\end{array}\right)
$$

and

$$
C^{0}=\left(\begin{array}{cc}
\exp (i \pi \hat{\alpha}) & \frac{-\hat{r} \pi}{\sin \pi \hat{\alpha}} \\
\frac{-\sin \pi \hat{\alpha}}{\pi \hat{r}} & \exp (i \pi \hat{\alpha})
\end{array}\right), \quad C^{1}=\left(\begin{array}{cc}
1 & \frac{\hat{r} \pi \exp (i \pi \hat{\alpha})}{\sin \pi \hat{\alpha}} \\
\frac{-\sin \pi \hat{\alpha} \exp (i \pi \hat{\alpha})}{\pi \hat{r}} & 1
\end{array}\right)
$$

We can factor out the diagonal matrix $D^{\hat{\alpha}}$ in $(2.65)$, and take $\hat{r}=1$. In this way, we obtain the formulae (2.45), (2.46), (2.47). The asymptotic behaviour of $y(x)$ can be computed as before. For $\sigma_{0}=0$ we obtain:

$$
y \sim a_{0} x \quad \text { for } \quad a_{0}=s
$$


This concludes the proof of the theorem.

2.2.5. The asymptotic behaviour near $1, \infty$ and the monodromy data. We can prove the analogue of Theorem 2.2 near 1 and $\infty$. Namely, for any pair of values $\left(a_{1}, \sigma_{1}\right)$ there exists a unique branch of the solution of PVI $\mu$ with the asymptotic behaviour

$$
y(x) \sim 1-a_{1} x^{1-\sigma_{1}} \quad \text { as } \quad x \rightarrow 1 .
$$

It is possible to parameterize the monodromy matrices as in Theorem 2.2 substituting $\sigma_{0}$ with $\sigma_{1}$ and $M_{0}$ with $M_{1}$ and vice-versa. Analogously, for any pair of values $\left(a_{\infty}, \sigma_{\infty}\right)$ there exists a unique branch of the solution of (PVI) with the asymptotic behaviour

$$
y(x) \sim a_{\infty} x^{\sigma_{\infty}} \quad \text { as } \quad x \rightarrow \infty
$$

and it is possible to parameterize the monodromy matrices as before, substituting $\sigma_{0}$ with $\sigma_{\infty}$ and applying the braid $\beta_{2}$ to the monodromy matrices.

\subsection{From the local asymptotic behaviour to the global one.}

In this section we prove Theorem 2.1 which gives the asymptotic behaviour of the branches of the solutions in terms of the triplets $\left(x_{0}, x_{1}, x_{\infty}\right)$.

Lemma 2.10. For the solution $y^{(0)}(x)$ of PVI $\mu$ behaving as

$$
y^{(0)}(x)=a_{0} x^{1-\sigma_{0}}\left(1+\mathcal{O}\left(x^{\varepsilon}\right)\right) \quad \text { as } \quad x \rightarrow 0,
$$

with $0 \leq \sigma_{0}<1$ and $a_{0} \neq 0, a_{0} \neq 1$ for $\sigma_{0}=0$, the canonical form (1.20) of the monodromy matrices $M_{0}^{(0)}, M_{x}^{(0)}, M_{1}^{(0)}$ given by (2.42), (2.41), (2.40), or (2.46), (2.47), (2.45) for $\sigma_{0}=0$, is the following:

$$
M_{0}=\left(\begin{array}{cc}
1 & -x_{0}^{(0)} \\
0 & 1
\end{array}\right), \quad M_{x}=\left(\begin{array}{cc}
1 & 0 \\
x_{0}^{(0)} & 1
\end{array}\right), \quad M_{1}=\left(\begin{array}{cc}
1+\frac{x_{1}^{(0)} x_{\infty}^{(0)}}{x_{0}^{(0)}} & -\frac{\left(x_{1}^{(0)}\right)^{2}}{x_{0}^{(0)}} \\
\frac{\left(x_{\infty}^{(0)}\right)^{2}}{x_{0}^{(0)}} & 1-\frac{x_{1}^{(0)} x_{\infty}^{(0)}}{x_{0}^{(0)}}
\end{array}\right)
$$

where the triple $\left(x_{0}^{(0)}, x_{1}^{(0)}, x_{\infty}^{(0)}\right)$ is defined, up to equivalence, by the following formulae, for $\sigma_{0} \neq 0$ :

$$
\begin{aligned}
& x_{0}^{(0)}=-2 \sin \frac{\pi \sigma_{0}}{2}, \\
& x_{1}^{(0)}=-\sqrt{2\left(\cos \pi \sigma_{0}-\cos 2 \pi \mu\right)} \frac{\sin \frac{\pi \phi}{2}}{\sin \frac{\pi \sigma_{0}}{2}}, \\
& x_{\infty}^{(0)}=-\sqrt{2\left(\cos \pi \sigma_{0}-\cos 2 \pi \mu\right)} \frac{\cos \frac{\pi\left(\sigma_{0}+\phi\right)}{2}}{\sin \frac{\pi \sigma_{0}}{2}},
\end{aligned}
$$

with $\phi$ given by

$$
e^{i \pi \phi}=\frac{1}{4 a_{0}} \frac{\sigma_{0}+2 \mu}{\sigma_{0}-2 \mu} \frac{\Gamma\left(1+\sigma_{0}\right)^{2} \Gamma\left(1-\frac{\sigma_{0}}{2}\right)^{2} \Gamma\left(1+\mu-\frac{\sigma_{0}}{2}\right) \Gamma\left(1-\mu-\frac{\sigma_{0}}{2}\right)}{\Gamma\left(1-\sigma_{0}\right)^{2} \Gamma\left(1+\frac{\sigma_{0}}{2}\right)^{2} \Gamma\left(1+\mu+\frac{\sigma_{0}}{2}\right) \Gamma\left(1-\mu+\frac{\sigma_{0}}{2}\right)},
$$


and for $\sigma_{0}=0$ :

$$
\begin{aligned}
& x_{0}^{(0)}=0, \\
& x_{1}^{(0)}=-|\sin \pi \mu| \sqrt{1-a_{0}} \\
& x_{\infty}^{(0)}=-|\sin \pi \mu| \sqrt{a_{0}} .
\end{aligned}
$$

The proof of this lemma can be obtained by straightforward computations, using the algorithm of lemma 1.5. Similar formulae for the parameters $\left(x_{0}^{(1)}, x_{1}^{(1)}, x_{\infty}^{(1)}\right)$ and $\left(x_{0}^{(\infty)}, x_{1}^{(\infty)}, x_{\infty}^{(\infty)}\right)$ can be obtained respectively starting from a solution $y^{(1)}(x)$ of $\mathrm{PVI} \mu$ behaving as

$$
y^{(1)}(x)=1-a_{1}(1-x)^{1-\sigma_{1}}\left(1+\mathcal{O}(1-x)^{\varepsilon}\right) \quad \text { as } \quad x \rightarrow 1,
$$

or from another solution $y^{(\infty)}(x)$ of $\mathrm{PVI} \mu$ behaving as

$$
y^{(\infty)}(x)=a_{\infty} x^{\sigma_{\infty}}\left(1+\mathcal{O}\left(\frac{1}{x^{\varepsilon}}\right)\right) \quad \text { as } \quad x \rightarrow \infty
$$

So, given an admissible triple $\left(x_{0}, x_{1}, x_{\infty}\right)$, with $x_{i} \in \mathbb{R},\left|x_{i}\right|<2$ for $i=0,1, \infty$, we choose the parameters $\mu,\left(a_{0}, \sigma_{0}\right),\left(a_{1}, \sigma_{1}\right)$ and $\left(a_{\infty}, \sigma_{\infty}\right)$ in such a way that $(2.8)$ is satisfied and

$$
x_{i}^{(0)}=x_{i}^{(1)}=x_{i}^{(\infty)}=x_{i}, \quad \text { for } \quad i=0,1, \infty .
$$

Using the explicit formulae (2.68), (2.69) for $x_{0} \neq 0$, we derive the expressions (2.10), (2.11). Similarly, using (2.70) for $x_{0}=0$ we derive the expression (2.12). In the same way, we derive the analogous expressions for $\left(a_{1}, \sigma_{1}\right)$ and $\left(a_{\infty}, \sigma_{\infty}\right)$. The three correspondent branches $y^{(0)}(x), y^{(1)}(x), y^{(\infty)}(x)$ of solutions of $\mathrm{PVI} \mu$, with $\mu$ given by (2.8) must coincide. In fact, the associated auxiliary Fuchsian systems have the same, modulo diagonal conjugation, monodromy matrices. This proves the existence of a solution of PVI $\mu$ with the asymptotic behaviour (2.1), with the indices given by (2.9) and the coefficients specified as above, for any admissible triple $\left(x_{0}, x_{1}, x_{\infty}\right)$, with $x_{i} \in \mathbb{R},\left|x_{i}\right|<2$ for $i=0,1, \infty$. The uniqueness of such a branch follows from theorem 1.3.

Conversely, for any such a solution we obtain an admissible triple $\left(x_{0}, x_{1}, x_{\infty}\right)=$ $\left(x_{0}^{(0)}, x_{1}^{(0)}, x_{\infty}^{(0)}\right)=\left(x_{0}^{(1)}, x_{1}^{(1)}, x_{\infty}^{(1)}\right)=\left(x_{0}^{(\infty)}, x_{1}^{(\infty)}, x_{\infty}^{(\infty)}\right)$, using the formulae $(2.68),(2.69)$ or (2.70) and their analogies. Let us prove that the numbers $\left(x_{0}, x_{1}, x_{\infty}\right)$ are real and satisfy $\left|x_{i}\right|<2$ for $i=0,1, \infty$. Indeed, from the definition of the parameters, it follows:

$$
\left(x_{0}^{(0)}\right)^{2}=4 \sin ^{2} \pi \sigma_{0},\left(x_{1}^{(1)}\right)^{2}=4 \sin ^{2} \pi \sigma_{1},\left(x_{\infty}^{(\infty)}\right)^{2}=4 \sin ^{2} \pi \sigma_{\infty} .
$$

This proves that our construction covers, for real $\mu$, all the solutions of $\mathrm{PVI} \mu$ with critical behaviour of algebraic type.

Finally, using corollary 1.5, we infer that the class of solutions of PVI $\mu$, with real $\mu$, having critical behaviour of algebraic type is invariant with respect to the analytic continuation. The law of transformation of the critical indices $l_{0}, l_{1}, l_{\infty}$ of the expansions (2.1), is described by theorem 1.5. 


\subsection{The complete list of algebraic solutions}

We summarize the results of this paper in the following

Classification Theorem. Any algebraic solution of the equation PVI $\mu$ with $2 \mu \notin \mathbb{Z}$ is equivalent, in the sense of symmetries (1.22), (1.23), (1.24) to one of the five solutions $\left(A_{3}\right),\left(B_{3}\right),\left(H_{3}\right),\left(H_{3}\right)^{\prime},\left(H_{3}\right)^{\prime \prime}$ below.

We already know that the classes of equivalent algebraic solutions are labelled by the five regular polyhedra and star-polyhedra in the three-dimensional space. We will construct representatives in these classes for the following values of the parameter $\mu$

$$
\mu=-\frac{1}{4}, \quad-\frac{1}{3}, \quad-\frac{2}{5}, \quad-\frac{1}{5}, \quad-\frac{1}{3} .
$$

The correspondent algebraic solutions will have 4, 3, 10, 10, 18 branches respectively. Recall that these are the lenghts of the orbits (1.33), (1.34), (1.35), (1.36), (1.37) respectively with respect to the action of the pure braid group (see remark 1.11 above). We give now the explicit formulae for the solutions with brief explanations of the derivations of them.

Tetrahedron. We have $\left(x_{0}, x_{1}, x_{\infty}\right)=(-1,0,-1)$, then $\mu=-\frac{1}{4}$ and

$$
\begin{aligned}
& y=\frac{(s-1)^{2}(1+3 s)\left(9 s^{2}-5\right)^{2}}{(1+s)\left(25-207 s^{2}+1539 s^{4}+243 s^{6}\right)}, \\
& x=\frac{(s-1)^{3}(1+3 s)}{(s+1)^{3}(1-3 s)} .
\end{aligned}
$$

(We present the solution in the parametric form). The monodromy matrices, in the canonical form (1.20), are:

$$
M_{0}=\left(\begin{array}{ll}
1 & 1 \\
0 & 1
\end{array}\right) \quad M_{x}=\left(\begin{array}{cc}
1 & 0 \\
-1 & 1
\end{array}\right) \quad M_{1}=\left(\begin{array}{ll}
1 & 0 \\
1 & 1
\end{array}\right) .
$$

This solution was found in [Dub] in the implicit form (E.29). This was also obtained, independently, by N. Hitchin (see [Hit2]). To reduce (E.29) to the above form, we have to solve the cubic equation (E.29 b) with the substitution:

$$
t=\frac{32\left(1-18 s^{2}+81 s^{4}\right)}{27\left(1+9 s^{2}+27 s^{4}+27 s^{6}\right)} .
$$

Then the three roots of (E.29 b) are:

$$
\begin{aligned}
\omega_{1} & =\frac{13-66 s^{2}-27 s^{4}}{3\left(1+3 s^{2}\right)^{2}} \\
\omega_{2,3} & =\frac{-5+42 s^{2} \pm 144 s^{3}+27 s^{4}}{3\left(1+3 s^{2}\right)^{2}} .
\end{aligned}
$$


Cube. We have $\left(x_{0}, x_{1}, x_{\infty}\right)=(-1,0,-\sqrt{2})$ and $\mu=-\frac{1}{3}$. The solution

$$
\begin{aligned}
& y=\frac{(2-s)^{2}(1+s)}{(2+s)\left(5 s^{4}-10 s^{2}+9\right)}, \\
& x=\frac{(2-s)^{2}(1+s)}{(2+s)^{2}(1-s)}
\end{aligned}
$$

was obtained in [Dub]. The canonical form for the monodromy matrices is:

$$
M_{0}=\left(\begin{array}{ll}
1 & 1 \\
0 & 1
\end{array}\right) \quad M_{x}=\left(\begin{array}{cc}
1 & 0 \\
-1 & 1
\end{array}\right) \quad M_{1}=\left(\begin{array}{cc}
1 & 0 \\
-2 & 1
\end{array}\right) .
$$

Coxeter group $\mathbf{W}\left(\mathbf{H}_{3}\right)$, of symmetries of icosahedron. We have three possible choices of the point $\left(x_{0}, x_{1}, x_{\infty}\right)$ which lead to three different solutions.

Icosahedron. The orbit (1.35) corresponds to the standard triple of reflections for the icosahedron. $\left(x_{0}, x_{1}, x_{\infty}\right)=\left(0,-1,-\frac{1+\sqrt{5}}{2}\right)$, then $\mu=-\frac{2}{5}$ and

$$
\begin{aligned}
& y=\frac{(s-1)^{2}(1+3 s)^{2}\left(-1+4 s+s^{2}\right)\left(7-108 s^{2}+314 s^{4}-588 s^{6}+119 s^{8}\right)^{2}}{(1+s)^{3}(-1+3 s) P(s)} \\
& x=\frac{(-1+s)^{5}(1+3 s)^{3}\left(-1+4 s+s^{2}\right)}{(1+s)^{5}(-1+3 s)^{3}\left(-1-4 s+s^{2}\right)}
\end{aligned}
$$

with

$$
\begin{aligned}
P(s)= & 49-2133 s^{2}+34308 s^{4}-259044 s^{6}+16422878 s^{8}-7616646 s^{10}+13758708 s^{12} \\
& +5963724 s^{14}-719271 s^{16}+42483 s^{18} .
\end{aligned}
$$

The canonical form for the monodromy matrices is:

$$
M_{0}=\left(\begin{array}{ll}
1 & 1 \\
0 & 1
\end{array}\right) \quad M_{x}=\left(\begin{array}{cc}
1 & 0 \\
-1 & 1
\end{array}\right) \quad M_{1}=\left(\begin{array}{cc}
1 & 0 \\
\frac{-3-\sqrt{5}}{2} & 1
\end{array}\right)
$$

The above solution was already obtained in [Dub] in the implicit form (E.33). The above explicit formula can be obtained solving (E.33 b) in the form:

$$
\begin{gathered}
t=\frac{\left(1-4 s-s^{2}\right)\left(-1-4 s+s^{2}\right)\left(-1+5 s^{2}\right)}{\left(1+3 s^{2}\right)^{3}} \\
\omega_{1}=\frac{25-585 s^{2}+3530 s^{4}-6690 s^{6}-3955 s^{8}+507 s^{10}}{\left(1+3 s^{2}\right)^{5}} \\
\omega_{2}=\frac{-7+215 s^{2}-1910 s^{4}-4096 s^{5}+5150 s^{6}+20480 s^{7}+6125 s^{8}-357 s^{10}}{\left(1+3 s^{2}\right)^{5}} \\
\omega_{3}=\frac{-7+215 s^{2}-1910 s^{4}+4096 s^{5}+5150 s^{6}-20480 s^{7}+6125 s^{8}-357 s^{10}}{\left(1+3 s^{2}\right)^{5} .}
\end{gathered}
$$


The last two solutions for the orbits (1.36) and (1.37), with the icosahedral symmetry are new. They correspond to great icosahedron and great dodecahedron respectively. To compute them we use the following algorithm. The leading terms of the Puiseux expansions near the ramification points $0,1, \infty$ of each branch can be computed by the formulae (2.9), (2.10), (2.11) and (2.12). From this the genus of the algebraic curve $F(y, x)=0$ is easily computed. Namely, the genus of (1.36) is 0 and the genus of (1.37) is 1 . Since the symmetries of $\operatorname{PVI} \mu$ preserve the indices $l_{0}, l_{1}, l_{\infty}$ (up to permutations), they preserve the genus too.

We observe that the appearance of genus 1 in the last solution related to the great dodecahedron could seem less surprising if we recall that the topology of this immersed two-dimensional surface is different from the topology of all the other polyhedra and starpolyhedra. In fact, this is a surface of genus 4, while all the others have genus 0 (see $[\mathrm{Cox}])$.

Let us now list the last two solutions.

Great Icosahedron. $\left(x_{0}, x_{1}, x_{\infty}\right)=\left(-1,0, \frac{1-\sqrt{5}}{2}\right)$, then $\mu=-\frac{1}{5}$ and

$$
\begin{aligned}
& y=\frac{(-1+s)^{4}(1+3 s)^{2}\left(-1+4 s+s^{2}\right)\left(3-30 s^{2}+11 s^{4}\right)^{2}}{(1+s)(-1+3 s)\left(1+3 s^{2}\right) P(s)}, \\
& x=\frac{(-1+s)^{5}(1+3 s)^{3}\left(-1+4 s+s^{2}\right)}{(1+s)^{5}(-1+3 s)^{3}\left(-1-4 s+s^{2}\right)} .
\end{aligned}
$$

with

$$
P(s)=\left(9-342 s^{2}+4855 s^{4}-28852 s^{6}+63015 s^{8}-1942 s^{10}+121 s^{12}\right) .
$$

The canonical form for the monodromy matrices is

$$
M_{0}=\left(\begin{array}{ll}
1 & 1 \\
0 & 1
\end{array}\right) \quad M_{x}=\left(\begin{array}{cc}
1 & 0 \\
-1 & 1
\end{array}\right) \quad M_{1}=\left(\begin{array}{cc}
1 & 0 \\
\frac{-3+\sqrt{5}}{2} & 1
\end{array}\right)
$$

Great Dodecahedron. $\left(x_{0}, x_{1}, x_{\infty}\right)=\left(-1,-1, \frac{1-\sqrt{5}}{2}\right), \mu=-\frac{1}{3}$. The canonical form for the monodromy matrices is

$$
M_{0}=\left(\begin{array}{ll}
1 & 1 \\
0 & 1
\end{array}\right) \quad M_{x}=\left(\begin{array}{cc}
1 & 0 \\
-1 & 1
\end{array}\right) \quad M_{1}=\left(\begin{array}{cc}
\frac{3-\sqrt{5}}{2} & 1 \\
\frac{-3+\sqrt{5}}{2} & \frac{1+\sqrt{5}}{2}
\end{array}\right)
$$

This is the most complicated solution and we will briefly explain how did we obtain it. As we already said, it is an algebraic function with 18 branches. It has two branch points of order 5 , two of order 3 and two regular branches, over every ramification 
point $0,1, \infty$. The branches $y_{1}(x), \cdots, y_{18}(x)$ near $x=0$ have the form:

$$
\begin{aligned}
y_{k}(x) & =e^{\frac{2 \pi i k}{5}}\left(\frac{7}{13}\right)^{2} 6^{\frac{-2}{5}} x^{\frac{4}{5}}+\mathcal{O}(x), \quad k=1, \cdots, 5 \\
y_{k+5}(x) & =e^{\frac{2 \pi i k}{5}} \frac{6^{\frac{4}{5}}}{19^{2}} x^{\frac{2}{5}}+\mathcal{O}\left(x^{\frac{4}{5}}\right), \quad k=1, \cdots, 5 \\
y_{10+k}(x) & =e^{\frac{2 \pi i k}{3}} \frac{2^{\frac{2}{3}}}{18} \frac{1+i \sqrt{15}}{4} x^{\frac{2}{3}}+\mathcal{O}(x), \quad k=1, \cdots, 3 \\
y_{13+k}(x) & =e^{\frac{2 \pi i k}{3}} \frac{2^{\frac{2}{3}}}{18} \frac{1-i \sqrt{15}}{4} x^{\frac{2}{3}}+\mathcal{O}(x), \quad k=1, \cdots, 3 \\
y_{17,18}(x) & =\frac{3 \pm \sqrt{5}}{6} x+\mathcal{O}\left(x^{2}\right) .
\end{aligned}
$$

The Puiseux expansions near $x=1$ and $x=\infty$ can be obtained from these formulae applying the symmetries (1.22) and (1.23) respectively. Using these formulae, one can compute any term of the Puiseux expansions of all the branches. Due to computer difficulties, at the moment, we do not manage to produce the explicit elliptic parameterization of the algebraic curve. We give this in the form of an algebraic curve of degree 36.

$$
x^{15} F(x, y, t)=0
$$

where

$$
t=x+\frac{1}{x}
$$

and

$$
\begin{gathered}
F(x, y, t)=\left(11423613917539180989-57169813730203944 t-13869163074392577 t^{2}\right. \\
\left.+1307302991918736 t^{3}-31962210377 t^{4}-556854952 t^{5}+282475249 t^{6}\right)^{2} x^{9}+ \\
+9(-42194267411458338799378785573556538817 \\
-58759262104428568315429822622247510492 t+ \\
+10095266581644469686796601774497789110 t^{2} \\
-969805106597038829472153249647160780 t^{3} \\
+13082239583395373581545441399627177 t^{4} \\
-77058446549850745165440956773416 t^{5} \\
-2150599531632473735225276196788 t^{6} \\
+5521397776112060589691860200 t^{7} \\
+34431689430132242698256649 t^{8}-4868379539328005204126748 t^{9} \\
+543298990997997546590 t^{10}-5420393254540081020 t^{11}
\end{gathered}
$$




$$
\begin{aligned}
& \left.-79792266297612001 t^{12}\right) x^{8}(1+x) y \\
& \text { +3 (1958540422461728364360419152068949457061 } \\
& -2209393132972329408615760780334959957197 t \\
& +3730909713982979160856372675878664915614 t^{2} \\
& -998456940153012666787445238400320871842 t^{3} \\
& +127635631698846877473536225998246411623 t^{4} \\
& -1482254497297246657880332587875322027 t^{5} \\
& +8847488219466307166390055942913100 t^{6} \\
& +115442527212405524632938663371228 t^{7} \\
& -1429542565820801871766655325509 t^{8} \\
& +7631245862019705473945545029 t^{9} \\
& +215859931310548879418863190 t^{10} \\
& -61369918621176379581242 t^{11} \\
& +443864075501101414537 t^{12} \\
& \left.+239376798892836003 t^{13}\right) x^{8} y^{2} \\
& \text { +8 (-14176454404869485146893293606614356611775 } \\
& +28055900200716580071561317517835005937542 t \\
& -22805214425386778204880821526748969126548 t^{2} \\
& +7203824414325261553629527716660045847442 t^{3} \\
& -941980967212371956151450075677431178469 t^{4} \\
& +8038149376577574092957422850094452382 t^{5} \\
& -22951481031624768208910085230213950 t^{6} \\
& -1567265072303229457696028497735362 t^{7} \\
& +17710721122570843039502588273105 t^{8} \\
& -100631536944878626686735036764 t^{9} \\
& -513222963217440801801106854 t^{10} \\
& +346131811374226777113368 t^{11} \\
& \left.-1385596068253512936373 t^{12}\right) x^{7}(1+x) y^{3} \\
& \text { +6 (372245126038285018174621123839906354129684 }
\end{aligned}
$$




$$
\begin{aligned}
& -538123164074188598920246979212739683700019 t \\
& +148097514409311992531878851512796911164392 t^{2} \\
& +131183971273631981690818920653550831952470 t^{3} \\
& -81255871706326021946002239342890704787124 t^{4} \\
& +13496764871847929345618085983834973142347 t^{5} \\
& -77522788387610267024773351181536807752 t^{6} \\
& -15773276759106249673670395386220524 t^{7} \\
& +43306225979803766149728090033557588 t^{8} \\
& -398429371182004923439978792917533 t^{9} \\
& +1762391142835398582269106251136 t^{10} \\
& +2388970787440139757429804470 t^{11} \\
& -3439831965306734927530580 t^{12} \\
& \left.+6227088023782555081845 t^{13}\right) x^{7} y^{4} \\
& \text { +6 (-1723593607166532080927038166074395946035900 } \\
& +2040599178888751273951239802196511605636025 t \\
& +233403452055579960658996997839662220718652 t^{2} \\
& -1266443503829326206558682114543567079698662 t^{3} \\
& +601472177228487289796456276889218593842200 t^{4} \\
& -90437995682218432847116536290883275511849 t^{5} \\
& +312726298388429638276816726813440288752 t^{6} \\
& +2550025722128829156352333577788952604 t^{7} \\
& -575104019542617989271403929336386084 t^{8} \\
& +4033434111720024901257683928676919 t^{9} \\
& -12117519775974826925705049828908 t^{10} \\
& -6556200250126220600244219830 t^{11} \\
& +14241314959493049268655304 t^{12} \\
& \left.-9850799841945252913527 t^{13}\right) x^{6}(1+x) y^{5} \\
& \text { +2 (21644465131825357400382632120971734649857264 } \\
& +1609281090760898028021044308156991213714259 t
\end{aligned}
$$




$$
\begin{aligned}
& -38819578264561008264384235926474662382590097 t^{2} \\
& +19461702669444598007359556763777303231431634 t^{3} \\
& +6809228919729818789589103927528063675916654 t^{4} \\
& -6502005871163632747227392004794985000703103 t^{5} \\
& +1176880351448958476049654953342289787508545 t^{6} \\
& -2305666451065939258487468463173953769764 t^{7} \\
& -66480299243646643579685794767909280748 t^{8} \\
& +15241200180683229312383060139285323669 t^{9} \\
& -78386104021612006912908252437015263 t^{10} \\
& +148880803913659745854808576095474 t^{11} \\
& +57461755817453559455002717038 t^{12} \\
& -103978838096755580164874073 t^{13} \\
& \left.+22079893897296692642367 t^{14}\right) x^{6} y^{6} \\
& \text { +12(-3846090734682844350682028823106084687428444 } \\
& \text {-6334186503545807261781842904989156398103475t } \\
& +13149149531444644750567430723972640952739601 t^{2} \\
& \text {-833478059474840476986699901359926517619482 } t^{3} \\
& -6957568559854570720866520753764257206265698 t^{4} \\
& +3704223179468379920674190362976879513996499 t^{5} \\
& -576109607175627912516018268715191958362489 t^{6} \\
& +449528751470785721434087640482632944192 t^{7} \\
& +97703132604175842587572789513022822852 t^{8} \\
& -16011119124352462144696375400709770865 t^{9} \\
& +57470033726311385832789992330535883 t^{10} \\
& -64186326557416461737056084783958 t^{11} \\
& -26094692352698228396829172726 t^{12} \\
& +25456979816863844482106097 t^{13} \\
& \left.-1051423518918890125827 t^{14}\right) x^{5}(1+x) y^{7} \\
& \text { +3 (10610560214390717981593236262575236159801442 }
\end{aligned}
$$


$+111851425974428655491946571184648885748780846 t$

$-46715489836280837819492546495478177251546881 t^{2}$

$-139030514021835704781706562226557058780091068 t^{3}$

$+91919803393449431080407081029445741045516160 t^{4}$

$+17286168898942635534811373112001322602942242 t^{5}$

$-24220067368877156108293128216367359106738667 t^{6}$

$+4637848726272650440828320863095758089676600 t^{7}$

$-768878602349792937649689712426759282462 t^{8}$

$-1741702208080601219941076960674171058190 t^{9}$

$+291817792658031512381548036217335383393 t^{10}$

$-703979549232767832584811827025318204 t^{11}$

$+431869052449224832223544125220252 t^{12}$

$+211026301709949401515207349982 t^{13}$

$\left.-88672767468343194407328645 t^{14}\right) x^{5} y^{8}$

$+(29516093700614758561713684532397059536112914$

$-250427363718702318175164789552712875171021018 t$

$-276026230387316277405019906885146213095909789 t^{2}$

$+798207668051094993868151955635553085940964248 t^{3}$

$-344467523172443772269951999752021715572380244 t^{4}$

$-120301086904813944940738235485125501727252554 t^{5}$

$+109511261517293051818507102282993534830623481 t^{6}$

$-19194080365623650515069714757340701033233072 t^{7}$

$-51032394918091643798626435084286655402518 t^{8}$

$+23520399409116636731567242035209841281394 t^{9}$

$-2981630391452674856104059990243312091171 t^{10}$

$+4599349671236813290177817558881705144 t^{11}$

$-1416023622121443571963532624008680 t^{12}$

$-884464824710945312016748613390 t^{13}$

$\left.+126470477973158698785100695 t^{14}\right) x^{4}(1+x) y^{9}$ 
+3 (-10911639347758887707980330460476045164474906

$-58595420545899882777261835025201788418411880 t$

$+510148671470006459933001332369933260295921507 t^{2}$

$-421411310692898883818707378130970229534690273 t^{3}$

$-167152535194408490298020642999503515413037404 t^{4}$

$+259430867087315198025819011038004422708530022 t^{5}$

$-46562493376530130089912033636689480134093531 t^{6}$

$-21317412351831533402564088853511437604716591 t^{7}$

$+5998321070650622801147765820299240777440702 t^{8}$

$+42318724644239526363080933921378429784628 t^{9}$

$-20025402467700581457477461594500061379211 t^{10}$

$+2543243908066676381203685445622311376505 t^{11}$

$-2382710944926354080442398446589552744 t^{12}$

$+326767340108407733203130499898126 t^{13}$

$+276491632581630260746191363123 t^{14}$

$\left.-8431365198210579919006713 t^{15}\right) x^{4} y^{10}$

+12 (-8733106988589310312964985955814462819079774

$+64016502559572741731505856556403573520814046 t$

$-144735875200699985710584864792377620711111027 t^{2}$

$+66707682241185015299381142924382044312819100 t^{3}$

$+73079504596828994404469155513764487920535332 t^{4}$

$-75764221368814908722894464652763782420369670 t^{5}$

$+18144429124108777614777763808360976174204563 t^{6}$

$+1935278311931502378271609920389021155764328 t^{7}$

$-842229927380965714885405569219684268010166 t^{8}$

$-43576284807293907660657241391770657495742 t^{9}$

$+12197493655076249609592887507484958732619 t^{10}$

$-1236906021392508491488029828633988502116 t^{11}$

$+656542960338462752927669545849000176 t^{12}$ 
$-33306604264547610372382676649434 t^{13}$

$\left.-41617303783672454910317327355 t^{14}\right) x^{3}(1+x) y^{11}$

+2 1187554318014748213259275472412247634165779260

$-762602529289184570716467664651505597594612711 t$

$+781137281074558579579982041608982979326338521 t^{2}$

+609916709974782293997005108542677011211842354t $t^{3}$

$-1300509731487820027375772452301766352965003310 t^{4}$

$+495021556835245340779707559257082787835603199 t^{5}$

$+115214340026017185187174532535570472667840767 t^{6}$

$-93151202230256791649185399844658098150754584 t^{7}$

$+7202343146876999296118334182261935450026080 t^{8}$

$+1570014403002611359784317587694467895480399 t^{9}$

$+371422610102654444897802704145750294582991 t^{10}$

$-107776513895628817503345944747365689465454 t^{11}$

$+10941070332070115448154615812894796283346 t^{12}$

$-3005301616251624310474021462023025751 t^{13}$

$+41932897601387821985740913526761 t^{14}$

$\left.+87168052345572726428101766292 t^{15}\right) x^{3} y^{12}$

+6 $(-49769698820686378083743112964571467299790844$

$+144208424244836145109900273916270122534480379 t$

$-31061701177281181816778329394184658654578125 t^{2}$

$-316115877280896274823885457480018099381675270 t^{3}$

$+413355491574586200245516861333774692564805758 t^{4}$

$-189323246544195194714568800051070616915145871 t^{5}$

$+16358105769812410561563772201680559507177601 t^{6}$

$+10699064629034472787428641330738981639504716 t^{7}$

$-2410111354335748769859016926649172233001216 t^{8}$

$+411171528032730820725099829153187243925077 t^{9}$

$-226789949801107593485509158636442297272707 t^{10}$ 


$$
\begin{gathered}
+47843156588060602804351104035547186862994 t^{11} \\
-4050842729096500910242692387785686802242 t^{12} \\
+503056665812777017090193080924335791 t^{13} \\
-1358120473689033753999092690625 t^{14} \\
\left.-4470156530542191098877013656 t^{15}\right) x^{2}(1+x) y^{13} \\
+6(40840375974497844675523416705994772589613816 \\
-31065278771086485580585566101107070670979537 t \\
-256058709793754221748465779158452450846012816 t^{2} \\
+559068508134217391316137877579221486889948317 t^{3} \\
-405356674135473476033247305191846353227354396 t^{4} \\
+56661024338479719866449660305901198060970619 t^{5} \\
+64125926679095609696574105582575413252584004 t^{6} \\
+
\end{gathered}
$$


$-1226684412907984419281022032089194096771900 t^{8}$

$-1114701349894370233505605371103641055314707 t^{9}$

$+706698148832598485833137372995728746006888 t^{10}$

$-230885597278675059768074093486733449982986 t^{11}$

$+40110760213781966306595755424591426952408 t^{12}$

$-2944406938738808019234484282441173992613 t^{13}$

$\left.+29909989810256194655311832623132956 t^{14}\right) x(1+x) y^{15}$

$+3(-19345311524103689299806429866595584344434933$

$+165880840018062517894524148661179410853072546 t$

$-433975351186661527899190510419861031681577223 t^{2}$

$+515516306674309051714096086492072331808918060 t^{3}$

$-283562876761607595979024343783955270990852289 t^{4}$

$+35089717870652037166528865782071242284918734 t^{5}$

$+33297928990127187049831304457387943687578909 t^{6}$

$-12917764244851664872827620472556082803226856 t^{7}$

$-266713623245328356955979252488258143292463 t^{8}$

$+555900198844440351814987030522263162652334 t^{9}$

$+344809125199575823496923125385565831315595 t^{10}$

$-325689072459807008457121908075371991483716 t^{11}$

$+117388439783020206894897144460070846332949 t^{12}$

$-21123688072686368568170196496753937437182 t^{13}$

$\left.+1569161588742434760282235480090100082255 t^{14}\right) x y^{16}$

+3 9783299760488948030219433006083570296689357

$-59321119347918543659930676521984384042169430 t$

$+141416477837529651726686264572772822193430055 t^{2}$

$-177096809878289456793903796377476455257673500 t^{3}$

$+127907586479651422318564410835908192786763365 t^{4}$

$-54372658309139640733439296021048049726746698 t^{5}$

$+13488394375983259178386269031077826541323679 t^{6}$ 


$$
\begin{aligned}
& -2113244794203694376441274534558687456380488 t^{7} \\
& +141785257824097311610019381070069013792095 t^{8} \\
& +316821091130460893567937727374441119017078 t^{9} \\
& -305480360931755555721215775431316200256739 t^{10} \\
& \text { +142141070595224470100170760768533902542116 } t^{11} \\
& -38457837145846954116338584809621985652097 t^{12} \\
& +5806436836462494743682658146129894324810 t^{13} \\
& \left.-380650359326333515862984779019865187923 t^{14}\right)(1+x) y^{17} \\
& +(-10524240525647109159259219575804205851284241 \\
& +54473554541130948618895564303322618142908415 t \\
& -116506041709060140591221129409148683447467665 t^{2} \\
& +134582692171688928175407226575844795641654895 t^{3} \\
& -91188532083602481766920967127844408926436405 t^{4} \\
& +36353592052137018784330858422674753425698363 t^{5} \\
& -7639385747972665348009342010125607135270845 t^{6} \\
& +93749989416978017153766638267237965058515 t^{7} \\
& +613514003480165484061014972915970847589645 t^{8} \\
& -277973572971202497026511206431230055671555 t^{9} \\
& +4186799108745525715968930085758736947197 t^{10} \\
& \text { +68475809505229552273919737578535787115805 } t^{11} \\
& -41025357958210023316522198089867194215575 t^{12} \\
& +12129433061687109251202289166065827342585 t^{13} \\
& -1903251796631667579314923895099325939615 t^{14} \\
& \left.+126883453108777838620994926339955062641 t^{15}\right) y^{18} \text {. }
\end{aligned}
$$




\section{BIBLIOGRAPHY}

[AgLak] R.P. Agarwal and V. Lakshmikantham, Uniqueness and Non-uniqueness Criteria for Ordinary Differential Equations, World Scientific (1993).

[Bat] H. Bateman and A. Erdelyi, Higher Transcendental Functions, Krieger Publishing, Florida (1981).

[Bir] J.S. Birman, Braids, Links, and Mapping Class Groups, Ann. Math. Stud. Princeton University (1975).

[Cox] H.S.M. Coxeter, Regular Polytopes, Macmillan Company New York (1963).

[Cro] W.J.R. Crosby, Problems and Solutions, Mounthly Amer. Math. (1946) 104-107.

[Dek] W. Dekkers, The Matrix of a Connection having Regular Singularities on a Vector Bundle of Rank 2 on $P^{1}(\mathbb{C})$, Springer Lect. Notes Math. 712 (1979) 33-43.

[Dub] B. Dubrovin, Geometry of 2D Topological Field Theories, Springer Lect. Notes Math. 1620, (1995) 120-348.

[FlN] H. Flashka and A.C. Newell, Monodromy and Spectrum Preserving Deformations, Comm. Math. Phys. 76 (1980) 67-116.

[Fuchs] R. Fuchs, Über Lineare Homogene Differentialgleichungen Zweiter Ordnung mit im drei im Endrichen Gelegene Wesentlich Singulären Stellen, Math. Ann. 63 (1907) 301-321.

[Gamb] B. Gambier, Sur les Equations Differentielles du Second Ordre et du Primier Degrè dont l'Integrale est a Points Critiques Fixes, Acta Math. 33, (1910) 1-55.

[Gar] R. Garnier, Sur des Equations Differentielles du Troisieme Ordre dont l'Integrale Generale est Uniforme et sur una Classe d'Equations nouvelles d'Ordre Superieur dont l'Integrale Generale a ses Points Critiques Fixes, Ann. Sci. Ecole Norm. Sup. 3 No.29 (1912) 1-126.

[Gor] P. Gordan, Uber endliche Gruppen linearer Transformationen einer Veranderlichen, Math. Ann. 12, 1877, 23-46.

[Hit] N.J. Hitchin, Twistor Spaces, Einstein Metrics and Isomonodromic Deformations, $J$. Differential Geometry 42, No.1 July 1995 30-112.

[Hit1] N.J. Hitchin, Poncelet Polygons and the Painlevé Transcendents, Geometry and Analysis, edited by Ramanan, Oxford University Press (1995) 151-185.

[Hit2] N.J. Hitchin, Talk at I.C.T.P. (Trieste) April 1993.

[IKSY] K. Iwasaki, H. Kimura, S. Shimomura, M. Yoshida, From Gauss to Painlevé, a Modern Theory for Special Functions, Chapter 4, Aspects of Mathematics, Vieweg (1991).

[Ince] E.L. Ince, Ordinary Differential Equations, Dover Publications, New York (1956).

[ItN] A.R. Its and V.Yu. Novokshenov, The Isomonodromic Deformation Method in the Theory of Painlevé Equations, Springer Lect. Notes Math. 1191 (1986). 
[Jim] M. Jimbo, Monodromy Problem and the Boundary Condition for some Painlevé Equations, Publ. RIMS 18 (1982), 1137-1161.

[JMU] M. Jimbo, T. Miwa and K. Ueno, Monodromy Preserving Deformation of the Linear Ordinary Differential Equations with Rational Coefficients I, II, Publ. RIMS 14 (1978) 223-267, 15 (1979) 201-278.

[Kit] A.V. Kitaev, Rational Solutions of the Fifth Painlevé Equation, Differential and Integral Equations 7 (1994) no.3-4, 967-1000.

[Kr] L. Kronecker, J. de Math. 19 (1854) 177-192.

[Lak] V. Lakshmikantham and S. Leela, Differential and Integral Inequalities, Academic Press New York (1969).

[Luka] N.A. Lukashevich, On the Theory of the Third Painlevé Equation, Differ. Uravn. 3 (1967) no.11, 1913-1923.

[Luke] L. Yu. Luke, Mathematical Functions and Their Approximations, Academic Press (1975).

[Mag] W. Magnus, Ring of Fricke Characters and Automorphism Groups of Free Groups, Math. Z. 170, (1980) 91-103.

[Mal] B. Malgrange, Sur les Deformations Isomonodromiques I, Singularités Régulières, Seminaire de l'Ecole Normale Superieure 1979-1982, Progress in Mathematics 37, Birkhäuser, Boston (1983) 401-426.

[Man] Yu.I. Manin, Sixth Painlevé Equation, Universal Elliptic Curve and Mirror of $\mathbf{P}^{2}$, preprint al-geom/9605010 (1996).

[Ma] M. Mazzocco, Picard and Chazy Solutions to PVI Equation, preprint 1998.

[Miwa] T. Miwa, Painlevé Property of Monodromy Preserving Deformation Equations and the Analyticity of $\tau$-function, Publ. RIMS, Kyoto Univ. 17 (1981) 703-721.

[Mur] Y. Murata, Classical Solutions of the Third Painlevé Equation, Nagoya Math. J. 139 (1995) 37-65.

[Mur1] Y. Murata, Rational Solutions of the Second and Fourth Painlevé equations, Funk. Ekvacioj 28, no.1 (1995).

[Nor] N.E. Nörlund, The Logarithmic Solutions of the Hypergeometric equation, Mat. Fys. Skr. Dan. Vid. Selsk. 2, no.5 (1963) 1-58.

[Ok] K. Okamoto, On the $\tau$-Function of the Painlevé Equations, Physica $2 D$ (1981) 525535 .

[Ok1] K. Okamoto, Studies on the Painlevé Equations I, Sixth Painlevé Equation, Ann. Mat. Pura Appl. 148 (1987) 337-381.

[Ok2] K. Okamoto, Painlevé Equations and Dynkin Diagrams, Painlevé Transcendents, edited by D. Levi and P. Winternitz, Plenum Press, New York (1992) 299-313. 
[Ok3] K. Okamoto, Sur les Feuilletages Associés aux Equation du Second Ordre à Points Critiques Fixes de Painlevé, Espaces des Conditions Initiales, Japan. J. Math. 5 (1979), 1-79.

[Pain] P. Painlevé, Sur les Equations Differentielles du Second Ordre et d'Ordre Superieur, dont l'Interable Generale est Uniforme, Acta Math. 25 (1902) 1-86.

[Pic] E. Picard, Mémoire sur la Théorie des Functions Algébriques de deux Varables, Journal de Liouville 5 (1889), 135-319.

[Seg] J. Segert, Frobenius Manifolds from Yang-Mills Instantons, preprint October (1997) dg-ga/9710031.

[SMJ] M. Sato, T. Miwa and M. Jimbo, Holonomic Quantum Fields II, Publ. RIMS 15 (1979) 201-278.

[Sch] L. Schlesinger, Über eine Klasse von Differentsial System Beliebliger Ordnung mit Festen Kritischer Punkten, J. fur Math. 141 (1912), 96-145.

[Schw] H.A. Schwartz, Über Diejenigen Fälle in Welchen die Gaussische Hypergeometrische Reihe einer Algebraische Funktion iheres vierten Elementes Darstellit, Crelle J. 75 (1873) 292-335.

[S1] S. Shimomura, Painlevé Transcendents in the Neighbourhood of a Fixed Singular Point, Funkcial. Ekvac. 25 (1982) 163-184.

[S2] S. Shimomura, Series Expansions for Painlevé Transcendents in the Neighbourhood of a Fixed Singular Point, Funkcial. Ekvac. 25 (1982) 185-197.

[S3] S. Shimomura, Supplement to "Series Expansions for Painlevé Transcendents in the Neighbourhood of a Fixed Singular Point", Funkcial. Ekvac. 25 (1982) 363-371.

[Sib] Y. Sibuya, Linear Differential Equations in the Complex Domain: Problems of Analytic Continuation, AMS TMM 82 (1990).

[Tod] K.P. Tod, Self-dual Einstein Metrics from the Painlevé VI Equation, Phys. Lett. A, 190 (1994) 221-224.

[Um] H. Umemura, Birational Automorphism Groups and Differential Equations, Nagoya Math. J., 119 (1990) 1-80.

[Um1] H. Umemura, Irreducebility of the First Differential Equation of Painlevé, Nagoya Math. J., 117 (1990) 231-252.

[Um2] H. Umemura, H. Watanabe, Solutions of the Second and Fourth Differential Equations of Painlevé, to appear in Nagoya Math. J., (1997).

[Um3] H. Umemura, H. Watanabe, Solutions of the Third Differential Equation of Painlevé, preprint (1997).

[Wat] H. Watanabe, Birational Canonical Transformations and Classical Solutions of the Sixth Painlevé Equation, preprint of the Kyushu University, Japan (1997). 
[Wat1] H. Watanabe, Solutions of the Fifth Painlevé Equation I, Hokkaido Math. J. XXIV, 2 (1995) p. 53-89.

[Wey] H. Weyl, Algebraic Theory of Numbers, Ann. Math. Stud. 1, Princeton University Press (1940). 